\title{
GENERAL COMBINATORIAL TOPOLOGY
}

\author{
BY \\ PAUL ALEXANDROFF \\ To Serge Bernstein on his sixtieth birthday
}

After the fundamental conceptions of the so-called combinatorial topology were transferred by the author of the present paper $\left(^{(1)}\right.$ as well as by Vietoris, Lefshetz, Cech and others to arbitrary compact metric spaces and, having obtained in the general duality law of Alexander-Pontrjagin, the homological theory of dimensionality and a number of other essentially new investigations a concrete geometrical development, became a mighty and generally recognized weapon in the investigation of different topological questions, it became not only possible to speak of a new branch of topology-the homological theory of spaces-but it also seemed that the directions of further development of this new branch were more or less determined. This latter opinion, however, was not confirmed: in 1934 Kolmogoroff $\left({ }^{1}\right)$ and nearly simultaneously with him Alexander $\left({ }^{2}\right)$ gave to the development of the homological topology an essentially new direction by the discovery of the so-called upper boundary operator (which we call here the $\nabla$-operator) dual to the old boundary operator (we call it here the $\Delta$-operator) and permitting one to construct two systems of homological invariants dual to each other in the sense of the Pontrjagin theory of characters not only for polyhedrons and complexes but also for arbitrary locally bicompact spaces.

The central fact of the theory is a proposition which was originally formulated by Kolmogoroff and which we therefore call in the present paper the duality law of Kolmogoroff. This proposition asserts that for any closed set $A$ lying in a locally bicompact space $R$, the $r$ - and $(r+1)$-dimensional Betti groups of which are null groups, there exists an isomorphism between the $r$-dimensional Betti group of $A$ and the $(r+1)$-dimensional Betti group of $R-A$. This proposition enables us to give a new meaning to the duality law

Presented to the Society, September 12, 1940; received by the editors April 6, 1940.

(1) Kolmogoroff, International Tensor Conference and International Topological Conference, Moscow, May, 1934, and September, 1935; papers: (a) Über die Dualität im Aufbau der kombinatorischen Topologie, Recueil Mathématique de Moscou, vol. 1 (43) (1936), pp. 97-102; (b) Les groupes de Betti des espaces localement bicompacts; Propriétés des groupes de Betti des espaces localement bicompacts; Les groupes de Betti des espaces metriques; Cycles relatifs, théor ème de dualité de M. Alexander-all four papers in the Comptes Rendus de l'Académie des Sciences, Paris, vol. 202 (1936), pp. 1144, 1325, 1558, 1641.

(2) Alexander (abstracted in the proceedings of the National Academy of Sciences for 1935): (a) On the connectivity ring of an abstract space, Annals of Mathematics, (2), vol. 37 (1936), pp. 698-708. (b) A theory of connectivity in terms of gratings, Annals of Mathematics, (2), vol. 39 (1938), pp. 883-912. The same ideas appear in a different form in the "pseudocycles" of Lefschetz (see his Topology) as soon as 1930. 
of Alexander-Pontrjagin (which may be easily deduced from the duality law of Kolmogoroff under the assumption that $R$ is the $n$-dimensional euclidean or spherical space).

In the construction of the theory itself Alexander and Kolmogoroff proceed differently. The construction of Kolmogoroff, a concise exposition of which without fundamental proofs was given by him in four notes in the Comptes Rendus de l'Académie des Sciences, Paris $\left({ }^{1}\right)$, is based on a completely new approach to homological problems of the set-theoretical topology and starts from the consideration of the functions $\phi^{r}\left(E_{0}, E_{1}, \cdots, E_{r}\right)$ and $f^{r}\left(e_{0}, e_{1}, \cdots, e_{r}\right)$, where the $E_{i}$ are sets and the $e_{i}$-points of the given space. The functions $\phi^{r}\left(E_{0}, E_{1}, \cdots, E_{r}\right)$ are skew-symmetrical and finitely additive with respect to all their arguments; their values belong to the bicompact commutative group $\Xi$ (the "field of coefficients"). The functions $f^{r}\left(e_{0}, e_{1}, \cdots, e_{r}\right)$ are also skew-symmetrical, but their values are taken from a discrete commutative group. The functions $\phi^{r}\left(E_{0}, E_{1}, \cdots, E_{r}\right)$ play the role of algebraical complexes of the usual combinatorial topology of complexes and are the starting point of the $\Delta$-theory. As analogues of algebraical complexes in the $\nabla$-theory appear not the functions $f^{r}\left(e_{0}, e_{1}, \cdots, e_{r}\right)$ themselves but classes of such functions equivalent to each other in a certain sense.

This way of construction may prove to be the most fruitful from the point of view of further investigations. But it considerably differs from the methods based on the elementary devices of combinatorial topology, which have dominated so far. This newness of the method as well as, undoubtedly, the fact that Kolmogoroff has not as yet given an exposition of the theory which is to any extent complete, nor, in particular, the proof of his duality law account, probably, for the fact that his theory is not yet as widespread as it deserves to be and that it has not so far influenced the further development of the topology to an extent to which it will doubtlessly influence it in the future.

Practically speaking, Alexander has realized his construction of the same theory in several different ways. A proof of the already mentioned duality law he gives, however, only in his last publication, $A$ theory of connectivity in terms of gratings $\left({ }^{2}\right)$, where to this end the whole theory is constructed on an entirely new basis with the help of the so-called gratings. The equivalence of this theory with the other theories of Alexander (as well as with the theory of Kolmogoroff) is not yet proved, although it is highly probable. The apparatus of gratings applied by Alexander in his last paper has a very simple geometrical figure (the decomposition of the space by a plane into two half-spaces) for its source. But in the general setting in which the construction proceeds this original figure becomes so complicated that the whole resulting structure is extremely involved.

In the present paper the theory is built on a completely elementary basis, namely by means of well known considerations of the finite coverings of the given space. I left my old devices of application of combinatorial methods 
to the study of general spaces only in one respect: along with the nerves of the coverings I consider now the barycentrical subdivisions of the coverings introduced by me recently elsewhere $\left({ }^{3}\right)$, which, as I think, give us of ten a more elastic weapon for the study of topological properties of the given space.

The distribution of the material is as follows. In the first two sections we construct the $\Delta$ - and the $\nabla$-theories for complexes. Here we systematize and prove things which are in the majority of cases known, but the proofs of which (and sometimes even the formulations) are, in a large proportion of cases, nowhere published $\left({ }^{4}\right)$.

In $\$ 3$ we recollect the notion of inverse and direct series of homomorphisms (instead of which we shall speak of direct and inverse spectra) of, in the general case partially ordered (not necessarily enumerable), systems of groups. This theory was originally constructed for enumerable sequences by Pontrjagin $\left({ }^{5}\right)$ and for arbitrary systems by Steenrod $\left({ }^{4}\right)$. I may point out the formulation of the conception of the limit group of a direct spectrum, which is logically simpler than the usual one.

In $\S 4$ we formulate and prove the "formal duality law" in application to arbitrary partially ordered systems of complexes. Substantially it is this "formal" duality that forms the combinatorial basis of the duality law of Kolmogoroff. The general formulation given here may prove convenient for application to the study of different concrete problems (local properties of sets, etc.).

In $\$ \S 5$ and 6 we prove the fundamental lemmas which shall be used in the proof of the duality law of Kolmogoroff. The distribution into two sections is made according to whether the lemmas concern finite coverings of an arbitrary set or special coverings of topological spaces. Thus the notion of the topological space we meet in the present paper for the first time in $\S 6$.

In $\$ 7$ we give the first definition of Betti groups for any spaces homeomorphic to open sets lying in normal spaces. This definition hangs together with my old papers as well as with the paper of Steenrod referred to above, i.e., it defines the Betti groups of a space as limit groups of respectively the direct and the inverse spectra composed of Betti groups of the nerves of finite coverings of the given space by its open sets. For the sequel it is, however, of importance that along with the Betti groups which for normal spaces were defined by Steenrod, we define also other groups taking particularly into account those elements of the covering, the closures of which are bicompact.

(3) Alexandroff, Diskrete Räume, Recueil Mathématique de Moscou, vol. 2 (44) (1937), pp. 501-518.

(4) See, however, Whitney, On matrices of integers and combinatorial topology, Duke Mathematical Journal, vol. 3 (1937), and On products in a complex, Annals of Mathematics, (2), vol. 39 (1938), pp. 397-432, as well as Steenrod, Universal homology groups, American Journal of Mathematics, vol. 58 (1936), pp. 661-701.

(5) Pontrjagin, Über den algebraischen Inhalt topologischer Dualitätssätze, Mathematische Annalen, vol. 105 (1931), pp. 165-205. 
It is just these groups, for the first time introduced in the present paper under the name of inner Betti groups, that form the object of the duality law of Kolmogoroff.

The same $\$ 7$ contains the proof of a theorem (Theorem 7.41), in which is formulated all that I know in the direction of the duality law of Kolmogoroff in the case when the space $R$ is not locally bicompact.

In $\$ 8$ we prove the duality law of Kolmogoroff for any locally bicompact normal space and closed $A \subset R$.

In $\$ 9$ is given a new definition of inner Betti groups based on the consideration of barycentrical subdivisions of finite coverings of $R$ by open sets and is proved the equivalence of this definition with the old one. At the end of this paragraph we give a formulation of the conception of Betti groups which does not use any auxiliary conceptions except the conception of the finite covering and the spectrum (series of homomorphisms) of groups.

In $\$ 10$ is introduced the operation of multiplication of elements of Betti groups and in this way the complete Betti group (i.e., the direct sum of Betti groups of different dimensionalities) is turned into a ring (the connectivity ring). Although this definition of multiplication follows that of Alexander $\left.{ }^{6}\right)$, it has in comparison with the latter an advantage consisting in the freedom from any special ordering of vertices which was applied in Alexander's paper On the connectivity ring of an abstract space $\left({ }^{2}\right)$, as well as in the freedom from any special conditions by means of which the multiplication is introduced in the paper $A$ theory of connectivity in terms of gratings $\left({ }^{2}\right)$.

\section{Notations}

Throughout this paper the following notations are of constant use:

(i) $A \cup B$ means the set theoretical sum, $A \cap B$ means the set theoretical intersection of the sets $A$ and $B$.

(ii) $\cup_{\alpha} A_{\alpha}$ means the set theoretical sum and $\cap_{\alpha} A_{\alpha}$ the intersection of all sets $A_{\alpha}$ of a given family of sets.

(iii) $A-B$ means the difference between the set $A$ and the set $B$, i.e., the set of all elements of $A$ not belonging to $B$. In this way

$$
A-B=A-A \cap B
$$

where is it not supposed that $B$ is a subset of $A$. But if $A$ is a commutative group and $B$ a subgroup of $A$, then $A-B$ means the factor (or difference) group of $A$ with respect to $B$.

(iv) $A \subset B$ means that every element of the set $A$ is an element of the set $B$ (the identity $A=B$ being not excluded).

(v) $a \varepsilon A$ means that $a$ is an element of the set $A$.

(6) Introduced in the paper On the connectivity ring of an abstract space (see footnote 2). See also in that paper the references to Cech and Whitney. 
(vi) If $t$ and $t^{\prime}$ are simplexes, then $t^{\prime}<t$ means that $t^{\prime}$ is a face of $t$. The letter $t$ denotes a non-oriented as well as an oriented simplex.

\section{Complexes}

1.1. By a complex we shall mean a finite simplicial complex $K$.

For any two oriented simplexes $t_{i}^{r}$ and $t_{j}^{r-1}$ of consecutive dimensionalities $r$ and $r-1$ of a complex $K$ we define the incidence coefficient $\left(t_{i}^{r}: t_{j}^{r-1}\right)$ as follows:

$1^{\circ}$. $\left(t_{i}^{r}: t_{j}^{r-1}\right)=0$, if $t_{j}^{r-1}$ is not a face of the simplex $t_{i}^{r}$.

$2^{\circ}$. Let the vertices of $t_{i}^{r}$ be $e_{0}, \cdots, e_{r}$ and the vertices of $t_{j}^{r-1}$ written in the order determining the given oriented simplex $t_{j}^{r-1}$ be $e_{i_{1}}, \cdots, e_{i_{r}}$, where $e_{i_{1}} \cdots, e_{i_{r}}$ are all vertices $e_{0}, \cdots, e_{r}$ with the exception of one, say $e_{k}$. Then

$$
\left(e_{k}, e_{i_{1}}, \cdots, e_{i_{r}}\right)=\epsilon t_{i}^{r},
$$

where, as may be easily seen, the coefficient $\epsilon= \pm 1$ depends only on the oriented simplex $t_{j}^{r-1}$ (and the oriented simplex $t_{i}^{r}$ ), but not on the special choice of the order $e_{i_{1}}, \cdots, e_{i_{r}}$ determining the oriented simplex $t_{j}^{r-1}$. We put

$$
\left(t_{i}^{r}: t_{j}^{r-1}\right)=\epsilon
$$

It is obvious that

$$
\left(-t_{i}^{r}: t_{j}^{r-1}\right)=\left(t_{i}^{r}:-t_{j}^{r-1}\right)=-\left(t_{i}^{r}: t_{j}^{r-1}\right) .
$$

Further, if $t_{i}^{r}=\left(e_{0}, \cdots, e_{r}\right)$ and $t_{j}^{r-1}=\left(e_{0}, \cdots, e_{k-1}, e_{k+1}, \cdots, e_{r}\right)$, then

$$
\left(t_{i}^{r}: t_{j}^{r-1}\right)=(-1)^{k} \text {. }
$$

1.2. The classical construction of combinatorial topology presupposes that a certain commutative group $J$ (generally speaking topological) is given, and is based on consideration of functions $f^{r}\left(t^{r}\right)$, the argument of which runs through the set of oriented simplexes $t^{r}$ of the complex $K$, and whose values are elements of the group $J$. Besides, it is assumed that always

$$
f^{r}\left(-t^{r}\right)=-f^{r}\left(t^{r}\right) \text {. }
$$

These functions, called the $r$-dimensional functions on the complex $K$ to the field of coefficients $J$ (also the $r$-dimensional algebraical complexes or the $r$-dimensional chains), form in virtue of the operation of addition defined in $J$ a commutative group $\operatorname{Lr}^{r}(K, J)$ which, if misunderstandings are excluded, we shall denote simply by $L^{r}(K)$.

The topology defined in $J$ defines a topology also in $L^{r}(K)$ : we obtain a neighbourhood of the element $f_{0}^{r} \varepsilon L^{r}(K)$ by choosing any neighbourhood $V$ of the zero element in $J$ and defining $V\left(f_{0}^{r}\right)$ as the set of all functions $f^{r}$ satisfying for all $t^{r} \varepsilon K$ the condition

$$
f^{r}\left(t^{r}\right)-f_{0}\left(t^{r}\right) \varepsilon V .
$$


1.3. The boundary operators : the lower operator $\Delta$ and the upper operator $\nabla$. To each element $f^{r}$ of the group $L^{r}(K)$ we correlate: the element $\Delta f^{r}$ of the group $L^{r-1}(K)$ and the element $\nabla f^{r}$ of the group $L^{r+1}(K)$ in the following manner:

$$
\begin{aligned}
& \Delta f^{r}\left(t^{r-1}\right)=\sum_{i}\left(t_{i}^{r}: t^{r-1}\right) f^{r}\left(t_{i}^{r}\right), \\
& \nabla f^{r}\left(t^{r+1}\right)=\sum_{i}\left(t^{r+1}: t_{i}^{r}\right) f^{r}\left(t_{i}^{r}\right),
\end{aligned}
$$

which in virtue of the adopted definition of incidence coefficients means

$$
\begin{aligned}
& \Delta f^{r}\left(e_{0}, \cdots, e_{r-1}\right)=\sum_{k} f^{r}\left(e_{k}, e_{0}, \cdots, e_{r-1}\right), \\
& \nabla f^{r}\left(e_{0}, \cdots, e_{r+1}\right)=\sum_{k}(-1)^{k} f^{r}\left(e_{0}, \cdots, \tilde{e}_{k}, \cdots, e_{r+1}\right),
\end{aligned}
$$

where, as always in the sequel, $\tilde{e}_{k}$ indicates that $e_{k}$ must be omitted. We have

$$
\Delta \Delta f^{r}=0 .
$$

In fact,

$$
\Delta \Delta f^{r}\left(e_{0}, \cdots, e_{r-2}\right)=\sum_{k} \Delta f^{r}\left(e_{k}, e_{0}, \cdots, e_{r-2}\right)=\sum_{h, k} f^{r}\left(e_{h}, e_{k}, e_{0}, \cdots, e_{r-2}\right) .
$$

But in the last sum for each term $f^{r}\left(e_{h}, e_{k}, e_{0}, \cdots, e_{r-2}\right)$ we can find a term

$$
f^{r}\left(e_{k}, e_{h}, e_{0}, \cdots, e_{r-2}\right)=-f^{r}\left(e_{h}, e_{k}, e_{0}, \cdots, e_{r-2}\right)
$$

such that their sum is equal to zero. Also

$$
\nabla \nabla f^{r}=0 .
$$

In fact,

$$
\begin{aligned}
& \nabla \nabla f^{r}\left(e_{0}, \cdots, e_{r+2}\right) \\
& =\sum_{k}(-1)^{k} \nabla f^{r}\left(e_{0}, \cdots, \tilde{e}_{k}, \cdots, e_{r+2}\right) \\
& =\sum_{k}(-1)^{k} \sum_{h<k}(-1)^{h} f^{r}\left(e_{0}, \cdots, \tilde{e}_{h}, \cdots, \tilde{e}_{k}, \cdots, e_{r+2}\right) \\
& \quad+\sum_{k}(-1)^{k} \sum_{h>k}(-1)^{h-1} f^{r}\left(e_{0}, \cdots, \tilde{e}_{k}, \cdots, \tilde{e}_{h}, \cdots, e_{r+2}\right)=0
\end{aligned}
$$

(since interchanging $h$ and $k$ we see that the terms of both sums differ only by the sign).

From the definition of the operators $\Delta$ and $\nabla$ it directly follows that they represent homomorphic mappings of $L^{r}(K, J)$ into $L^{r-1}(K, J)$ and into $L^{r+1}(K, J)$. In the case of a topological group $J$ these homomorphisms are continuous. 
DEFINITION 1.33. If $\Delta f^{r}=0$, then the function $f^{r}$ is called $a \Delta$-cycle; if $\nabla f^{r}=0$, then the function $f^{r}$ is called $a \nabla$-cycle.

In this terminology the theorems $(1.32 \Delta)$ and $(1.32 \nabla)$ may be formulated as follows: the $\Delta$ - and $\nabla$-boundaries of every function are respectively $\Delta$-and $\nabla$-cycles.

Definition 1.34. The cycle $f^{r}$ is said to be homologous to zero on $K$ if there exists a function of which $f^{r}$ is the boundary (of corresponding denotation, i.e. $\Delta \operatorname{or} \nabla)$.

From the above follows: the $r$-dimensional $\Delta$-cycles form the kernel of the homomorphism $\Delta$ of the group $L^{r}(K)$ into the group $L^{r-1}(K)$. They form the group $Z_{\Delta}^{r}(K, J)$ (or simply $Z_{\Delta}^{r}(K)$ ). Similarly, the $r$-dimensional $\nabla$-cycles form the group $Z_{\nabla}^{r}(K, J)$ or $Z_{\nabla}^{r}(K)$ - the kernel of the homomorphic mapping $\nabla$ of the group $L^{r}(K)$ into the group $L^{r+1}(K)$. Hence and from the continuity of the homomorphisms $\Delta$ and $\nabla$ it follows that $Z_{\Delta}^{r}(K)$ and $Z_{\nabla}^{r}(K)$ are closed subgroups of the group $L^{r}(K)$.

The image of the group $L^{r}(K)$ under the homomorphism $\Delta$ is the group $H^{r-1}(K, J)$ or $H_{\Delta}^{r-1}(K)$ of all $(r-1)$-dimensional $\Delta$-cycles homologous to zero in $K$. Similarly, the image of the group $L^{r}(K)$ under the homomorphism $\nabla$ is the group $H_{\nabla}^{+1}(K, J)$ or $H_{\nabla}^{++1}(K)$ of all $(r+1)$-dimensional $\nabla$-cycles homologous to zero in $K$.

If the group $J$ is bicompact, then so are the groups $L^{r}(K)$ and also its closed subgroups $Z_{\Delta}^{r}(K)$ and $Z_{\nabla}^{r}(K)$. The groups $H_{\Delta}^{r}(K)$ and $H_{\nabla}^{r}(K)$, being images of bicompact groups $L^{r+1}(K)$ and $L^{r-1}(K)$ under continuous homomorphisms $\Delta$ and $\nabla$ are bicompact and, consequently, closed in $Z_{\Delta}^{r}(K)$ and $Z_{\nabla}^{r}(K)$ respectively.

1.4. Definition 1.4. The factor groups

$$
\begin{aligned}
& Z_{\Delta}^{r}(K, J)-\bar{H}_{\Delta}^{r}(K, J)=B_{\Delta}^{r}(K, J), \\
& Z_{\nabla}^{r}(K, J)-\bar{H}_{\nabla}^{r}(K, J)=B_{\nabla}^{r}(K, J)
\end{aligned}
$$

(where $\bar{H}$ is the closure of the group $H$ in the group $Z$ ) are called respectively the $r$-dimensional $\Delta$-and $\nabla$-Betti groups of the complex $K$ to the field of coeffcients $J$. If $J$ is bicompact or discrete, then obviously $\bar{H}=H$.

Henceforth we shall consider only bicompact and discrete fields of coefficients.

1.5. Let $X$ and $\Xi$ be two commutative groups dual in the sense that each of them is the group of characters of the other (see the Addendum at the end of the paper). Let $X$ be discrete and $\Xi$ bicompact. We consider the functions $f^{r}$ and $\phi^{r}$ belonging respectively to $L^{r}(K, X)$ and $L^{r}(K, \Xi)$ and introduce the following notations: 
DEFinition 1.51. (The scalar products of functions.)

$$
\phi^{r} f^{r}=\sum_{t_{i}^{r} \varepsilon K} \phi^{r}\left(t_{i}^{r}\right) f^{r}\left(t_{i}^{r}\right) .
$$

Correlating to every function $\phi^{r} \varepsilon L^{r}(K, \Xi)$ the element $\phi^{r} f^{r} \varepsilon \kappa$, we obtain for a given fixed $f^{r} \varepsilon L^{r}(K, X)$ a homomorphic mapping of the group $L^{r}(K, \Xi)$ into $\kappa$. In other words, every $f^{r} \varepsilon L^{r}(K, X)$ may be considered as a character of the group $L^{r}(K, \Xi)$.

Let us prove that, conversely, every character of the group $L^{r}(K, \Xi)$ is generated in this sense by an element of the group $L^{r}(K, X)$.

Let a character $h$ of the group $L^{r}(K, \Xi)$ be given. Take any simplex $t_{i}^{r} \varepsilon K$ and consider those functions $\phi^{r} \varepsilon L^{r}(K, \Xi)$ which only on $t_{i}^{r}$ are different from zero. The set of these functions completed by the function identically equal to zero forms a subgroup $L_{i}^{r}$ of the group $L^{r}(K, \Xi)$ isomorphic to the group $\Xi$. The subgroup $L_{i}^{r}$ is mapped by the homomorphism $h$ into $\kappa$ and this mapping may be considered in virtue of the just established isomorphism between $L_{t}^{r}$ and $\boldsymbol{\Xi}$ as an element

$$
f_{i}^{h}=f^{r}\left(t_{i}^{r}\right)
$$

of the group $X$. Thus a function

$$
f^{r} \varepsilon L^{r}(K, X)
$$

is defined.

For any $\phi_{i}^{r} \varepsilon L_{i}^{r}$ we have

$$
\dot{\phi}_{i}^{r}\left(r_{i}^{r}\right) f^{r}\left(\dot{t}_{i}^{r}\right)=\phi_{i}^{r}\left(t_{i}^{r}\right) f_{i}^{h}=h \phi_{i}^{r} \varepsilon \kappa .
$$

If $\phi^{r}$ is an arbitrary element of the group $L^{r}\left(K, \Xi^{\prime}\right)$ and if $\phi_{i}^{r}$ is the function from $L_{i}^{r}$ coinciding with $\phi^{r}$ on $t_{i}^{r}$ and equal to zero on all other simplexes from $K$, then

$$
\phi^{r} f^{r}=\sum_{i} \phi^{r}\left(t_{i}^{r}\right) f^{r}\left(t_{i}^{r}\right)=\sum_{i} \phi_{i}^{r}\left(t_{i}^{r}\right) f^{r}\left(t_{i}^{r}\right)=\sum_{i} h \phi_{i}^{r}=h \sum_{i} \phi_{i}^{r}=h \phi^{r},
$$

q.e.d. We have thus proved the following:

THEOREM 1.521. The group $\operatorname{Lr}^{r}(K, X)$ is the group of characters of the group $L^{r}(K, \Xi)$.

Quite similarly is proved

THEOREM 1.522. The group $L^{r}(K, \Xi)$ is the group of characters of the group $L^{r}(K, X)$.

THEOREM 1.53. The homomorphisms $\Delta$ and $\nabla$ are conjugate homomor- 
phisms of the group $L^{r}(K, \Xi)$ into the group $L^{r-1}(K, \Xi)$ and of the group $L^{r-1}(K, X)$ into the group $L^{r}(K, X)$.

In other words

$$
\phi^{r} \nabla f^{r-1}=\Delta \phi^{r} f^{r-1}
$$

In fact,

$$
\begin{aligned}
\phi^{r} \nabla f^{r-1} & =\sum_{i} \phi^{r}\left(t_{i}^{r}\right) \sum_{j}\left(t_{i}^{r}: t_{j}^{r-1}\right) f^{r-1}\left(t_{j}^{r-1}\right)=\sum_{i, j}\left(t_{i}^{r}: t_{j}^{r-1}\right) \phi^{r}\left(t_{i}^{r}\right) f^{r-1}\left(t_{j}^{r-1}\right) \\
& =\sum_{i} f^{r-1}\left(t_{j}^{r-1}\right) \sum_{i}\left(t_{i}^{r}: t_{j}^{r-1}\right) \phi\left(t_{i}^{r}\right)=\sum_{j} f^{r-1}\left(t_{j}^{r-1}\right) \Delta \phi^{r}\left(t_{j}^{r-1}\right) \\
& =f^{r-1} \Delta \phi^{r},
\end{aligned}
$$

q.e.d.

1.6. Definition 1.60. Let $M$ be any subset of the complex $K$ (i.e., a set whose elements are simplexes of the complex $K$ ). We shall say that the function $f^{r} \varepsilon L^{r}(K, J)$ lies on $M$ and write $f^{r} \subset M$, if for every simplex $t^{r} \subset K-M$ we have $f^{r}\left(t^{r}\right)=0$.

The sum of two functions lying on $M$ evidently lies also on $M$.

In the sequel $M$ will always be either a subcomplex $Q$ of the complex $K$ or the set $K-Q$ complementary to a subcomplex $Q \subset K$.

DeFinition 1.61. The group of all $r$-dimensional functions $f^{r} \varepsilon L^{r}(K)$ lying on $K-Q$ will be denoted by $L^{r}(K-Q)$. The group of all $r$-dimensional $\nabla$-cycles lying on $K-Q$ will be denoted by $Z^{r}(K-Q)$.

Evidently

$$
Z_{\nabla}^{r}(K-Q)=Z_{\nabla}^{r}(K) \cap L^{r}(K-Q) \subset L^{r}(K) .
$$

1.611. If $f^{r} \cdot \varepsilon L^{r}(K-Q)$, then

$$
\nabla f^{r} \varepsilon Z_{\nabla}^{r+1}(K-Q) \text {. }
$$

This assertion follows directly from the fact that all faces of a simplex belonging to $Q$ also belong to $Q$.

Definition 1.612. $A \nabla$-cycle fr $\varepsilon Z^{r}(K-Q)$ is said to be homologous to zero on $K-Q$,

$$
f^{r} \sim 0 \text { on } K-Q
$$

if it is the $\nabla$-boundary of a function

$$
f^{r-1} \varepsilon L^{r-1}(K-Q) \text {. }
$$

The $r$-dimensional $\nabla$-cycles lying on $K-Q$ and homologous to zero on 
$K-Q$ form the subgroup $H_{\nabla}^{r}(K-Q)$ of the group $Z^{r}(K-Q)$ (and of the group $\left.H_{\nabla}^{r}(K)\right)$.

The factor group

$$
Z_{\nabla}^{r}(K-Q)-H_{\nabla}^{r}(K-Q)
$$

will be denoted by $B_{\nabla}^{r}(K-Q, J)$, or simply by $B_{\nabla}^{r}(K-Q)$.

DeFinition 1.62. Let again $Q$ be a subcomplex of $K$. The function $f^{r}$ is said to be a $\Delta$-cycle modulo $Q$, if $\Delta f^{r} \subset Q$.

The $r$-dimensional $\Delta$-cycles modulo $Q$ evidently form a group; we shall denote it by $Z_{\Delta}^{r}(K \bmod Q)$. We have

$$
Z_{\Delta}^{r}(K \bmod Q) \supset Z_{\Delta}^{r}(K) .
$$

Definition 1.621. Let $f^{r}$ be a cycle modulo $Q$. If there exists a function $f^{r+1}$ such that

$$
\Delta f^{r+1}=f^{r}+u^{r}, \quad u^{r} \subset Q,
$$

then we shall say that $f^{r}$ is homologous to zero modulo $Q$ on $K$,

$$
f^{r} \sim 0 \text { modulo } Q \text { on } K \text {. }
$$

The $r$-dimensional $\Delta$-cycles modulo $Q$ homologous to zero on $K$ modulo $Q$ form a subgroup $H_{\Delta}^{r}(K \bmod Q)$ of the group $Z_{\Delta}^{r}(K \bmod Q)$. We have

$$
H_{\Delta}^{r}(K \bmod Q) \supset H_{\Delta}^{r}(K) \text {. }
$$

The factor group

$$
Z_{\Delta}^{r}(K \bmod Q)-H_{\Delta}^{r}(K \bmod Q)
$$

is denoted by $B_{\Delta}^{r}(K \bmod Q)$ (or by $\left.B_{\Delta}^{r}(K \bmod Q, J)\right)$.

Let again $X$ and $\Xi$ be two groups dual to each other, $X$ discrete and $\Xi$ bicompact.

THEOREM 1.63. The annihilator of the group $H_{\nabla}^{r}(K-Q, X)$ in the group $L^{r}(K, \Xi)$ is the group $Z_{\nabla}^{r}(K \bmod Q, \Xi)$.

We have to prove two assertions:

$1^{\circ}$. If $\phi^{r} \varepsilon Z_{\Delta}^{r}(K \bmod Q, \Xi), f^{r} \subset H_{\nabla}^{r}(K-Q, X)$, then $\phi^{r} f^{r}=0$.

$2^{\circ}$. If $\phi^{r} \varepsilon L^{r}(K, \Xi)$ is a function not belonging to $Z_{\Delta}^{r}(K \bmod Q, \Xi)$, then there exists such an $f^{r} \varepsilon H_{\nabla}^{r}(K-Q, X)$ that $\phi^{r} f^{r} \neq 0$.

Proof of $1^{\circ}$. By assumption

$$
f^{r}=\nabla f^{r-1}, f^{r-1} \varepsilon L^{r-1}(K-Q, X) ;
$$

then 


$$
\phi^{r} f^{r}=\phi \nabla f^{r-1}=\Delta \phi^{r} f^{r-1}=\sum_{j} \Delta \phi^{r}\left(x_{j}^{r-1}\right) f^{r-1}\left(x_{j}^{r-1}\right)=0 .
$$

Proof of $2^{\circ}$. Suppose that $\phi^{r}$ does not enter into $Z_{\Delta}^{r}(K \bmod Q)$. Then there exist such a $t_{1}^{r-1} \varepsilon K-Q$ that

$$
\Delta \phi^{r}\left(x_{1}^{r-1}\right)=\alpha \neq 0,
$$

$\alpha \varepsilon \Xi$

Choose an $a \varepsilon X$ such that $\alpha a \neq 0$ and put

$$
\begin{aligned}
& f^{r-1}\left(t_{1}^{r-1}\right)=a, \\
& f^{r-1}\left(t_{i}^{r-1}\right)=0 \text { for } t_{i}^{r-1} \varepsilon K, \quad i \neq 1 .
\end{aligned}
$$

Then obviously $f^{r-1} \varepsilon L^{r-1}(K-Q)$. Putting $f^{r}=\nabla f^{r-1}$, we have

$$
\begin{gathered}
f^{r} \varepsilon H_{\nabla}^{r}(K-Q, X), \\
\phi^{r} f^{r}=\phi^{r} \nabla f^{r-1}=\Delta \phi^{r} f^{r-1}=\Delta \phi^{r}\left(t_{1}^{r}\right) f^{r-1}\left(t_{1}^{r-1}\right)=\alpha a \neq 0,
\end{gathered}
$$

and Theorem 1.63 is proved.

THEOREM 1.64. The annihilator of the group $H_{\Delta}^{r}(K \bmod Q, \Xi)$ in the group $L^{r}(K, X)$ is the group $Z_{\nabla}^{r}(K-Q, X)$.

Proof. We prove, firstly: if

$$
\phi^{r} \varepsilon H_{\Delta}^{r}(K \bmod Q, \Xi), \quad f^{r} \varepsilon Z_{\nabla}^{r}(K-Q, X),
$$

then

$$
\phi^{r} f^{r}=0 \text {. }
$$

In fact,

$$
\begin{aligned}
\phi^{r} & =\Delta \phi^{r+1}+\psi^{r}, & \psi^{r} \subset Q, \\
\phi^{r} f^{r} & =\Delta \phi^{r+1} f^{r}+\psi^{r} f^{r}=\phi^{r+1} \nabla f^{r}=0 . &
\end{aligned}
$$

We prove, secondly: if the function $f^{r} \varepsilon L^{r}(K, X)$ is not a cycle lying on $K-Q$, then there exists a function $\phi^{r} \varepsilon H_{\Delta}^{r}\left(K \bmod Q\right.$, 西) such that $\phi^{r} f^{r} \neq 0$.

(a) Let $f^{r}$ be any function not lying on $K-Q$. Then there exists a $t_{1}^{r} \varepsilon Q$ such that $f^{r}\left(t_{1}^{r}\right)=a \neq 0$. Take such an $\alpha \varepsilon \Xi$ that $\alpha a \neq 0$ and put

$$
\begin{aligned}
& \phi^{r}\left(t_{1}^{r}\right)=\alpha, \\
& \phi^{r}\left(t_{i}^{r}\right)=0 \text { on other } \quad t_{i}^{r} \varepsilon K .
\end{aligned}
$$

Then we have

$$
\begin{gathered}
\phi^{r} \varepsilon H_{\Delta}^{r}(K \bmod Q, \Xi), \\
\phi^{r} f^{r}=\phi^{r}\left(t_{1}^{r}\right) f^{r}\left(t_{1}^{r}\right)=\alpha a \neq 0 .
\end{gathered}
$$


(b) Let $f^{r} \varepsilon L^{r}(K, X)$ lie on $K-Q$ and not be a $\nabla$-cycle. Then for a certain $t_{1}^{r+1} \varepsilon K-Q$ we have $\nabla f^{r}\left(t_{1}^{r+1}\right)=a \neq 0$. Choose $\alpha \varepsilon \Xi$ such that $\alpha a \neq 0$ and put

$$
\begin{aligned}
& \phi^{r+1}\left(t_{1}^{r+1}\right)=\alpha, \\
& \phi^{r+1}\left(t_{i}^{r+1}\right)=0 \text { for other } t_{i}^{r+1} \varepsilon K .
\end{aligned}
$$

Then

$$
\begin{gathered}
\Delta \phi^{r+1} \varepsilon H_{\Delta}^{r}(K, \Xi) \subset H_{\Delta}^{r}(K \bmod , \Xi), \\
\Delta \phi^{r+1} f^{r}=\phi^{r+1} \nabla f^{r}=\phi^{r+1}\left(t_{1}^{r+1}\right) \nabla f^{r}\left(t_{1}^{r+1}\right)=\alpha a \neq 0,
\end{gathered}
$$

and Theorem 1.64 is proved.

Putting in Theorems 1.63 and 1.64

$$
Q=0
$$

we have

1.631. The annihilator of the group $H_{\nabla}^{r}(K, X)$ in the group $\operatorname{Lr}^{r}(K, \Xi)$ is the group $Z_{\Delta}^{r}(K, \Xi)$.

1.641. The annihilator of the group $H_{\Delta}^{r}(K, \Xi)$ in the group $\operatorname{Lr}^{r}(K, X)$ is the group $Z_{\Delta}^{r}(K, X)$.

From the theory of characters it is known that if two groups $G$ and $\Gamma$ are dual to each other and if the subgroup $\Gamma_{0} \subset \Gamma$ is the annihilator in $\Gamma$ of the subgroup $G_{0} \subset G$, then, conversely, $G_{0}$ is the annihilator in $G$ of the group $\Gamma_{0}$. On ground of this remark we deduce from 1.63 and 1.64

1.632. The annihilator of the group $Z_{\Delta}(K \bmod Q, \Xi)$ in $L^{r}(K, X)$ is $H_{\nabla}^{r}(K-Q, X)$.

1.642. The annihilator of the group $Z_{\nabla}^{r}(K-Q, X)$ in $L^{r}(K, \Xi)$ is $H_{\Delta}^{r}(K \bmod Q, \Xi)$.

1.7. Let $A, B, C$ be three commutative groups, all three discrete or all three bicompact, and let

$$
A \supset B, \quad A-B=C .
$$

If we denote the groups of characters of the groups $A, B, C$ respectively by $A^{\prime}, B^{\prime}, C^{\prime}$, then one of the fundamental theorems of the theory of characters may be formulated as follows: $C^{\prime}$ is a subgroup of $A^{\prime}$, namely the annihilator of the group $B$ in the group $A^{\prime}$, and

$$
B^{\prime}=A^{\prime}-C^{\prime} \text {. }
$$

Or the annihilator of the subgroup $B \subset C$ in $A^{\prime}$ is $C^{\prime}$. On ground of this theorem we deduce from 1.63 and 1.64 
1.73. The group $Z_{\Delta}^{r}(K \bmod Q, \Xi)$ is the group of characters of the group $L^{r}(K, X)-H_{\nabla}^{r}(K-Q, X)$.

1.74. The group $Z_{\nabla}^{r}(K-Q, X)$ is the group of characters of the group $L^{r}(K, \Xi)-H_{\Delta}^{r}(K \bmod Q, \Xi)$.

In the same way from 1.642 and 1.632 we deduce

1.731. The group $H_{\Delta}^{r}(K \bmod Q, \Xi)$ is the group of characters of the group $L^{r}(K, X)-Z_{\nabla}^{r}(K-Q, X)$.

1.741. The group $H_{\nabla}^{r}(K-Q, X)$ is the group of characters of the group $L^{r}(K, \Xi)-Z_{\nabla}^{r}(K \bmod Q, \Xi)$.

Recall now the so-called second theorem on isomorphisms of Emmy Noether: If for commutative groups $U, V, W$ we have the inclusion relations $U \supset V \supset W$, then considering (in a manner easily understood) $V-W$ as a subgroup of the group $U-V$, we have

$$
(U-W)-(V-W) \approx U-V
$$

(where $\approx$ means isomorphic).

Putting

$$
U=L^{r}(K, \Xi), \quad V=Z_{\Delta}^{r}(K \bmod Q, \Xi), \quad W=H_{\Delta}^{r}(K \bmod Q, \Xi),
$$

we find

$$
\begin{aligned}
{\left[L^{r}(K, \Xi)-H_{\Delta}^{r}(K \bmod Q, \Xi)\right]-Z_{\Delta}^{r}(K \bmod Q, \Xi) } & -H_{\Delta}^{r}(K \bmod Q, \Xi) \\
& \approx L^{r}(K, \Xi)-Z_{\Delta}^{r}(K \bmod Q, \Xi),
\end{aligned}
$$

wherefrom, on ground of 1.72 ,

$\chi\left[Z_{\Delta}^{r}(K \bmod Q, \Xi)-H_{\Delta}^{r}(K \bmod Q, \Xi)\right]=\chi\left[L^{r}(K, \Xi)-H_{\Delta}^{r}(K \bmod Q, \Xi)\right]$

$$
-\chi\left[L^{r}(K, \Xi)-Z_{\Delta}^{r}(K \bmod Q, \Xi)\right] \text {, }
$$

and on ground of 1.74 and 1.741 ,

$$
\chi\left[Z_{\Delta}^{r}(K \bmod Q, \Xi)-H_{\Delta}^{r}(K \bmod Q, \Xi)\right] \approx Z_{\Delta}^{r}(K-Q, X)-H_{\nabla}^{r}(K-Q, X),
$$
i.e.,

$$
\chi\left[B_{\Delta}^{r}(K \bmod Q, \Xi)\right]=B_{\nabla}^{r}(K-Q, X) .
$$

Putting $Q=0$ we obtain as a special case of formula (1.75)

Theorem 1.751. The Betti groups $B_{\Delta}^{r}(K, \Xi)$ and $B_{\nabla}^{r}(K, X)$ are dual to each other.

1.8. Consider separately the case $r=0$. 
Since a zero-dimensional $\nabla$-cycle cannot be homologous to zero, $B_{\nabla}^{0}(K)$ $=Z_{\nabla}^{0}(K)$.

Let $K$ be an arbitrary complex. Every function $f^{0}$ constant on $K$ (i.e., assuming for all null-dimensional simplexes $t^{0} \varepsilon K$ one and the same value) is a $\nabla$-cycle.

In fact, for every one-dimensional simplex $t^{1}=\left(e_{0}, e_{1}\right)$ we have

$$
\nabla f^{0}\left(t^{1}\right)=f^{0}\left(e_{1}\right)-f^{0}\left(e_{0}\right)=0 .
$$

1.80. If $K$ is connected and $f^{0} \varepsilon Z_{\nabla}^{0}(K)$, then $f^{0}$ is constant on $K$.

In fact, if $f^{0}$ is not constant on $K$, then in virtue of the connectivity of $K$ we may find a $t^{1}=\left(e_{0}, e_{1}\right)$ such that $f^{0}\left(e_{0}\right) \neq f^{0}\left(e_{1}\right)$ and, consequently, $\nabla f^{0}\left(t^{1}\right) \neq 0$.

Thus on connected complexes the zero-dimensional constant functions and only they are cycles.

Hence follows

1.81. For every complex $K$ the group $Z_{\nabla}^{0}(K)$ consists of those and only those zero-dimensional functions which are constant on every component of $K$.

1.82. The group $B_{\nabla}^{0}(K, J)$ is a direct sum of groups isomorphic to $J$ and the number of direct summands in this sum is equal to the number of components of the complex $K$.

In the group $Z_{\nabla}^{0}(K)=B_{\nabla}^{0}(K)$ is contained the subgroup $Z_{\nabla}^{00}(K)$ of those cycles which are constant on the whole $K$. The factor group

$$
Z_{\nabla}^{0}(K)-Z_{\nabla}^{00}(K)
$$

we shall denote by $B_{\nabla}^{00}(K)$.

1.83. If $K$ consists of $p$ components, then $B_{\nabla}^{00}(K, J)$ is a direct sum of $p-1$ groups each of which is isomorphic to the group $J$.

Defining the group $Z_{\Delta}^{00}(K)$ as the group of those zero-dimensional functions, the sum of values of which extended over all vertices of $K$ is equal to zero, we have in the group $Z_{\Delta}^{00}(K)$ a subgroup $H_{\Delta}^{0}(K)$ of all zero-dimensional $\Delta$-cycles homologous to zero. The group $B_{\Delta}^{00}(K)=Z_{\Delta}^{00}(K)-H_{\Delta}^{0}(K)$ is, as is well known, also a direct sum of $p-1$ groups isomorphic to $J$ and, consequently, is isomorphic to the group $B_{\nabla}^{00}(K)$. The groups $B_{\nabla}^{00}(K, X)$ and $B_{\Delta}^{00}(K, \Xi)$ are obviously dual to each other.

\section{Simplicial MAPPINGS OF COMPLEXES}

2.1. Suppose that to every vertex $e^{\beta}$ of a complex $K^{\beta}$ corresponds the vertex $S e^{\beta}=e^{\alpha}$ of a complex $K^{\alpha}$ such that to vertices belonging to any simplex of the complex $K^{\beta}$ correspond vertices belonging to a simplex of the complex $K^{\alpha}$. This correspondence of vertices establishes a mapping $S$ of the complex $K^{\beta}$ 
into the complex $K^{\alpha}$ : to every simplex $t_{\beta}=\left(e_{0}^{\beta}, \cdots, e_{\tau}^{\beta}\right)$ of the complex $K^{\beta}$ corresponds a simplex $S t_{\beta}$ of the complex $K^{\alpha}$ with vertices $S e_{0}^{\beta}, \cdots, S e_{r}^{\beta}$ and the number of dimensions of $S t_{\beta}$ is less than or equal to the number of dimensions of the simplex $t_{\beta}$. The so-obtained mapping is called a simplicial mapping of the complex $K^{\beta}$ into the complex $K^{\alpha}$.

In virtue of the mapping $S$, to an oriented simplex $t_{\beta}=\left(e_{0}^{\beta}, \cdots, e_{r}^{\beta}\right)$ of the complex $K^{\beta}$ corresponds an oriented simplex (which may be degenerate)

$$
t_{\alpha}=S t_{\beta}=\left(S e_{0}^{\beta}, \cdots, S e_{r}^{\beta}\right)
$$

of a complex $K^{\alpha}$ of the same number of dimensions as $t_{\beta}$.

2.2. A simplicial mapping $S$ of the complex $K^{\beta}$ into the complex $K^{\alpha}$ generates

$1^{\circ}$. A homomorphic mapping $\rho$ of the group $L^{r}\left(K^{\beta}\right)$ into the group $L^{r}\left(K^{\alpha}\right)$.

$2^{\circ}$. A homomorphic mapping $\sigma$ of the group $L^{r}\left(K^{\alpha}\right)$ into the group $L^{r}\left(K^{\beta}\right)$.

Indeed, to every function $f_{\beta}^{r} \varepsilon L^{r}\left(K^{\beta}\right)$ corresponds a function $\rho f_{\beta}^{\gamma} \varepsilon L^{r}\left(K^{\alpha}\right)$ defined by

$$
\rho f_{\beta}^{r}\left(t_{\alpha}^{r}\right)=\sum f^{r}\left(t_{\beta}^{r}\right),
$$

where the summation is extended over all $t_{\beta}^{r} \varepsilon K^{\beta}$ such that $S t_{\beta}^{r}=t_{\alpha}^{r}$. To every function $f_{\alpha}^{r} \varepsilon L^{r}\left(K^{\alpha}\right)$ corresponds a function $\sigma f_{\alpha}^{r} \varepsilon L^{r}\left(K^{\beta}\right)$ defined by

$$
\sigma f_{\alpha}^{r}\left(t_{\beta}^{r}\right)=f^{r}\left(S t_{\beta}^{r}\right) .
$$

THEOREM 2.211. The homomorphism $\rho$ preserves the lower boundary operator $\Delta$ :

$$
\Delta \rho f_{\beta}^{r}=\rho \Delta f_{\beta}^{r}
$$

In fact,

$$
\begin{aligned}
\Delta \rho f_{\beta}^{r}\left(e_{1}^{\alpha}, \cdots, e_{r}^{\alpha}\right) & =\sum_{i} \rho f_{\beta}^{r}\left(e_{i}^{\alpha}, e_{1}^{\alpha}, \cdots, e_{r}^{\alpha}\right) \\
& =\sum_{i} \sum_{j, j_{1}, \cdots, j_{r}} f_{\beta}^{r}\left(e_{j}^{\beta}, e_{j_{1}}^{\beta}, \cdots, e_{j_{r}}^{\beta}\right),
\end{aligned}
$$

where the summation in the inner sum is extended over all such $j, j_{1}, \cdots, j_{r}$ that $S e_{j}^{\beta}=e_{i}^{\alpha}, S e_{j_{1}}^{\beta}=e_{1}^{\alpha}, \cdots, S e_{j_{r}}^{\beta}=e_{r}^{\alpha}$. Hence

$$
\begin{aligned}
\Delta \rho f_{\beta}^{r}\left(e_{1}^{\alpha}, \cdots, e_{r}^{\alpha}\right) & =\sum_{j_{1}, \cdots, i_{r}} \sum_{j} f_{\beta}^{r}\left(e_{j}^{\beta}, e_{j_{1}}^{\beta}, \cdots, e_{j_{r}}^{\beta}\right) \\
& =\sum_{j_{1}, \cdots, j_{r}} \Delta f_{\beta}^{r}\left(e_{j_{1}}^{\beta}, \cdots, e_{j_{r}}^{\beta}\right)=\rho \Delta f_{\beta}^{r}\left(e_{1}^{\alpha}, \cdots, e_{r}^{\alpha}\right) .
\end{aligned}
$$

THEOREM 2.221. The homomorphism $\sigma$ preserves the upper boundary operator $\nabla$ : 


$$
\nabla \sigma f^{r}=\sigma \nabla f^{r}
$$

In fact, we have the inequalities

$$
\begin{aligned}
\nabla \sigma f_{\alpha}^{r}\left(e_{0}^{\beta}, \cdots, e_{r+1}^{\beta}\right) & =\sum_{k}(-1)^{k} \sigma f_{\alpha}^{r}\left(e_{0}^{\beta}, \cdots, \bar{e}_{k}^{\beta}, \cdots, e_{r+1}^{\beta}\right) \\
& =\sum_{k}(-1)^{k} f_{\alpha}^{r}\left(S e_{0}^{\beta}, \cdots, \bar{e}_{k}, \cdots, S e_{r+1}^{\beta}\right) \\
& =\nabla f_{\alpha}^{r}\left(S e_{0}^{\beta}, \cdots, S e_{r+1}^{\beta}\right) \\
& =\sigma \nabla f_{\alpha}^{r}\left(e_{0}^{\beta}, \cdots, e_{r+1}^{\beta}\right) .
\end{aligned}
$$

2.3. Let two sub-complexes $Q^{\alpha} \subset K^{\alpha}$ and $Q^{\beta} \subset K^{\beta}$ be given and let the simplicial mapping $S$ be such that $S Q^{\beta} \subset Q^{\alpha}$. Then from

follows

$$
f_{\beta}^{\tau} \subset Q^{\beta}
$$

and from

$$
\rho f_{\beta}^{r} \subset Q^{\alpha}
$$

follows

$$
f_{\alpha}^{r} \subset K^{\alpha}-Q^{\alpha}
$$

$$
\sigma f_{\alpha}^{r} \subset K^{\beta}-Q^{\beta}
$$

Therefore we have the inclusion relations

$$
\begin{gathered}
\rho Z_{\Delta}^{r}\left(K^{\beta} \bmod Q^{\beta}\right) \subset Z_{\Delta}^{r}\left(K^{\alpha} \bmod Q^{\alpha}\right), \\
\rho H_{\Delta}^{r}\left(K^{\beta} \bmod Q^{\beta}\right) \subset H_{\Delta}^{r}\left(K^{\alpha} \bmod Q^{\alpha}\right), \\
\sigma Z_{\nabla}^{\gamma}\left(K^{\alpha}-Q^{\alpha}\right) \subset Z_{\nabla}^{r}\left(K^{\beta}-Q^{\beta}\right), \\
\sigma H_{\nabla}^{r}\left(K^{\alpha}-Q^{\alpha}\right) \subset H_{\nabla}^{r}\left(K^{\beta}-Q^{\beta}\right) .
\end{gathered}
$$

THEOREM 2.33. The homomorphism $\rho$ generates the homomorphic mapping $\tilde{\omega}$ of the group $B_{\Delta}^{r}\left(K^{\beta} \bmod Q^{\beta}\right)$ into the group $B_{\Delta}^{r}\left(K^{\alpha} \bmod Q^{\alpha}\right)$; the homomorphism $\sigma$ generates the homomorphic mapping $\pi$ of the group $B_{\nabla}^{r}\left(K^{\alpha}-Q^{\alpha}\right)$ into the group $B_{\nabla}^{r}\left(K^{\beta}-Q^{\beta}\right)$.

2.4. Let, as always, $\Xi$ and $X$ be two dual groups, $\Xi$ bicompact, $X$ discrete.

THEOREM 2.41. The homomorphic mapping $\rho$ of the group $L^{r}\left(K^{\beta}, \Xi\right)$ into the group $L^{r}\left(K^{\alpha}, \Xi\right)$ and the homomorphic mapping $\sigma$ of the group $L^{r}\left(K^{\alpha}, \Xi\right)$ into the group $L^{r}\left(K^{\beta}, X\right)$ are conjugated.

In fact, for any $\phi_{\beta}^{r} \varepsilon L^{r}\left(K^{\beta}, \Xi\right)$ and $f_{\alpha}^{r} \varepsilon L^{r}\left(K^{\alpha}, X\right)$,

$$
\dot{\phi}_{\beta}^{r} \sigma f_{\alpha}^{r}=\sum_{j} \dot{\phi}_{\beta}^{r}\left(t_{\beta j}^{r}\right) f_{\alpha}^{r}\left(S t_{\beta j}^{r}\right),
$$


or, denoting by

$$
\sum_{i}^{i}
$$

the summation over all $t_{\beta j}^{r}$ such that $S t_{\beta j}^{r}=t_{\alpha t}^{r}$ (and zero, if such $t_{\beta j}^{r}$ do not exist),

$$
\begin{aligned}
\dot{\phi}_{\beta}^{r} \sigma f_{\alpha}^{r} & =\sum_{i}\left[\sum_{j}^{i} \phi_{\beta}^{r}\left(t_{\beta j}^{r}\right)\right] f_{\alpha}^{r}\left(t_{\alpha i}^{r}\right) \\
& =\sum_{i} \rho f_{\beta}^{r}\left(t_{\alpha i}^{r}\right) f_{\alpha}^{r}\left(t_{\alpha i}^{r}\right)=\rho f_{\beta}^{r} \cdot f_{\alpha}^{r} .
\end{aligned}
$$

THEOREM 2.42. The homomorphic mapping $\tilde{\omega}$ of the group $B_{\Delta}^{r}\left(K^{\beta} \bmod Q^{\beta}, \Xi\right)$ into the group $B_{\Delta}^{r}\left(K^{\alpha} \bmod Q^{\alpha}, \Xi\right)$ and the homomorphic mapping $\pi$ of the group $B_{\nabla}^{r}\left(K^{\beta}-Q^{\beta}, X\right)$ into the group $B_{\nabla}^{r}\left(K^{\alpha}-Q^{\alpha}, X\right)$ are conjugated.

In fact, let $\zeta^{\beta} \varepsilon B_{\Delta}^{r}\left(K^{\beta} \bmod Q^{\beta}, \Xi\right), z^{\alpha} \varepsilon B_{\nabla}^{\tau}\left(K^{\alpha}-Q^{\alpha}, X\right)$ be chosen arbitrarily. Choose the cycles $\phi_{\beta}^{r} \varepsilon \zeta^{\beta}$ and $f_{\alpha}^{r} \varepsilon z^{\alpha}$. Then $\tilde{\omega} \zeta^{\beta}$ is an element of the group $B_{\Delta}^{r}\left(K^{\alpha} \bmod Q^{\alpha}, \Xi\right)$ containing $\rho \phi_{\beta}^{r}$ and $\pi z^{\alpha}$ is an element of the group $B_{\nabla}^{\tau}\left(K^{\beta}-Q^{\beta}, X\right)$ containing $\sigma f_{\alpha}^{\gamma}$. We have

$$
\zeta^{\beta} \cdot \pi z^{\alpha}=\phi_{\beta}^{r} \cdot \sigma f_{\alpha}^{r}=\rho \phi_{\beta}^{r} \cdot f_{\alpha}^{r}=\tilde{\omega} \zeta^{\beta} \cdot z^{\alpha},
$$

q.e.d. From the result just proved directly follows

2.43. Let $S$ and $S^{\prime}$ be two simplicial mappings of the complex $K^{\beta}$ into the complex $K^{\alpha}$ such that for given subcomplexes $Q^{\beta} \subset K^{\beta}$ and $Q^{\alpha} \subset K^{\alpha}$ we have $S\left(Q^{\beta}\right) \subset Q^{\alpha}, S^{\prime}\left(Q^{\beta}\right) \subset Q^{\alpha}$. Let both mappings $S$ and $S^{\prime}$ generate one and the same homomorphism $\tilde{\omega}$ of the group $B_{\Delta}^{r}\left(K^{\beta} \bmod Q^{\beta}, \Xi\right)$ into the group $B_{\Delta}^{r}\left(K^{\alpha} \bmod Q^{\alpha}, \Xi\right)$. Then the mappings $S$ and $S^{\prime}$ generate one and the same homomorphism $\pi$ of the group $B_{\nabla}^{r}\left(K^{\alpha}-Q^{\alpha}, X\right)$ into the group $B_{\nabla}^{r}\left(K^{\beta}-Q^{\beta}, X\right)$.

The dual formulation is, of course, also true.

The following remark is essential for the sequel. Let the simplicial mappings $S_{0}$ and $S_{1}$ of the complex $K^{\beta}$ into the complex $K^{\alpha}$ satisfy the condition

2.44. Whatever be the simplex $t_{\beta} \subset K^{\beta}$ there exists a simplex $t_{\alpha} \subset K^{\alpha}$ having among its faces the simplexes $S_{0} t_{\beta}$ and $S_{1} t_{\beta}$ and if $t_{\beta} \subset Q^{\beta}$, then we may suppose that $t_{\alpha} \subset Q^{\alpha}$.

In this case the mappings $S_{0}$ and $S_{1}$ are evidently homotopic and if we denote by $S_{u}, 0 \leqq u \leqq 1$, the deformation of $S_{0}$ into $S_{1}$, we may suppose that for any $u$ we have $S_{u} \bar{Q}^{\beta} \subset \bar{Q}^{\alpha}$. Hence it follows that $S_{0}$ and $S_{1}$ generate one and the same homomorphism $\tilde{\omega}$ of the group $B_{\Delta}^{r}\left(K^{\beta} \bmod Q^{\beta}, \Xi\right)$ into the group $B_{\Delta}^{r}\left(K^{\alpha} \bmod Q^{\alpha}, \Xi\right)$. From 2.43 it follows that $S_{0}$ and $S_{1}$ generate one and the same homomorphism $\pi$ of the group $B_{\nabla}^{r}\left(K^{\alpha}-Q^{\alpha}, X\right)$ into the group $B_{\nabla}^{r}\left(K^{\beta}-Q^{\beta}, X\right)$.

2.45. If the simplicial mappings $S_{0}$ and $S_{1}$ satisfy the condition 2.44 , then 
they generate one and the same homomorphism $\tilde{\omega}$ of the group $B_{\Delta}^{r}\left(K^{\beta} \bmod Q^{\beta}, \Xi\right)$ into the group $B_{\Delta}^{r}\left(K^{\alpha} \bmod Q^{\alpha}, \Xi\right)$ and one and the same homomorphism $\pi$ of the group $B_{\nabla}^{r}\left(K^{\alpha}-Q^{\alpha}, X\right)$ into the group $B_{\nabla}^{r}\left(K^{\beta}-Q^{\beta}, X\right)\left({ }^{7}\right)$.

\section{SPECTRA AND THEIR LIMIT GROUPS}

3.1. A partially ordered set $D$ shall be called unbounded, if for any two elements $d_{1}$ and $d_{2}$ of the set $D$ there exists an element $d_{3}$ following after $d_{1}$ as well as after $d_{2}$ :

$$
d_{3}>d_{1}, d_{3}>d_{2} \text {. }
$$

Consider the unbounded partially ordered set $\Delta$ consisting of the groups $H^{\alpha}$; for the sake of simplicity we shall suppose that the indices $\alpha$ are ordinal numbers (the order of which, however, must not be at all connected with the order in $\Delta$ ). Suppose that for any two groups $H^{\alpha}$ and $H^{\beta}$ which are elements of $\Delta$ and satisfy in $\Delta$ the condition $H^{\beta} \geqq H^{\alpha}$ a homomorphic mapping $\tilde{\omega}_{\alpha}^{\beta}$ (projection) of the group $H^{\beta}$ in to the group $H^{\alpha}$ is defined such that for $H^{\gamma} \geqq H^{\beta} \geqq H^{\alpha}$ we have always

$$
\tilde{\omega}_{\alpha}^{\beta} \tilde{\omega}_{\beta}^{\gamma}=\tilde{\omega}_{\alpha}^{\gamma}
$$

and $\tilde{\omega}_{\alpha}^{\beta}$ is the identical mapping.

The system of groups $H^{\alpha}$ and mappings $\tilde{\omega}_{\alpha}^{\beta}$ is called the inverse spectrum and is denoted by $\left[H^{\alpha} ; \tilde{\omega}_{\alpha}^{\beta}\right]$.

Every inverse spectrum defines the limit group

$$
H=\lim _{\leftarrow}\left[H^{\alpha} ; \tilde{\omega}_{\alpha}^{\beta}\right] \text {. }
$$

The elements of the group $H$ are the threads of the spectrum $\left[H^{\alpha} ; \tilde{\omega}_{\alpha}^{\beta}\right]$, i.e., systems of elements $\eta=\left\{\eta^{\alpha}\right\}$ satisfying the following conditions:

$1^{\circ}$. Every $\eta^{\alpha}$ is an element of the group $H^{\alpha}$ and $\eta$ contains only one element of each group.

$2^{\circ}$. If $\eta^{\alpha}$ and $\eta^{\beta}$ are elements of the thread $\eta$ and $\eta^{\alpha} \varepsilon H^{\alpha}, \eta^{\beta} \varepsilon H^{\beta}$, then $\tilde{\omega}_{\alpha}^{\beta} \eta^{\beta}=\eta^{\alpha}$.

If $\eta_{1}=\left\{\eta_{1}^{\alpha}\right\}$ and $\eta_{2}=\left\{\eta_{2}^{\alpha}\right\}$ are two threads of the inverse spectrum $\left[H^{\alpha} ; \tilde{\omega}_{\alpha}^{\beta}\right]$, then $\eta=\left\{\eta_{1}^{\alpha}+\eta_{2}^{\alpha}\right\}$ is also a thread and we put

$$
\eta=\eta_{1}+\eta_{2} \text {. }
$$

Fixing $\alpha$ and correlating to every thread $\eta$ the element $\eta^{\alpha}$ contained in it, we obtain a homomorphic mapping $\tilde{\omega}_{\alpha}$ of the group $H$ into the group $H^{\alpha}$. The inverse spectra are considered always under the assumption that the groups $H^{\alpha}$ are bicompact. Then the group $H=\lim _{\leftarrow}\left[H^{\alpha} ; \tilde{\omega}_{\alpha}^{\beta}\right]$ will be also topologized;

(7) A purely combinatorial proof of this theorem has been given by Čech, Théorie générale de l'homologie, Fundamenta Mathematicae, vol. 19 (1932), pp. 149-183, especially pp. 158-159 $(\S 12)$. 
a neighbourhood of the thread $\eta_{0}=\left\{\eta_{0}^{\alpha}\right\}$ is obtained, if we choose a finite number $\eta_{0}^{\alpha_{i}}, \cdots, \eta_{0}^{\alpha_{s}}$ of its elements, choose for each of them a neighbourhood $O \eta_{0}^{\alpha_{1}}$ in $H^{\alpha_{i}}$ and take all threads $\eta=\left\{\eta^{\alpha}\right\}$ satisfying the conditions $\eta^{\alpha_{i}} \varepsilon O \eta_{0}^{\alpha_{i}}$ for $i=1,2, \cdots, s$. The so-topologized group $H$ proves to be bicompact.

3.2. Suppose that we have an unbounded partially ordered set $D$ of discrete groups $\mathcal{F}^{\alpha}$ and assume, further, for convenience that all elements $h^{\alpha}$ of the group $\mathfrak{F C}^{\alpha}$ are different from the elements $h^{\beta}$ of the group $\mathfrak{F}^{\beta}$, if $\alpha \neq \beta$.

Suppose further that for any two groups $\mathfrak{F C}^{\alpha}, \mathfrak{H C}^{\beta}$ such that $\mathfrak{F}^{\alpha}<\mathcal{F}^{\beta}$ in $D$ is established a homomorphic mapping $\pi_{\beta}^{\alpha}$ (projection) of the group $\mathfrak{F}^{\alpha}$ into the group $\mathcal{F}^{\beta}$ such that for $\mathcal{F}^{\alpha} \leqq \mathcal{F C}^{\beta} \leqq \mathcal{F C}^{\gamma}$ we have $\pi_{\gamma}^{\beta} \pi_{\beta}^{\alpha}=\pi_{\gamma}^{\alpha}$ and $\pi_{\alpha}^{\alpha}$ is the identical mapping. The system of groups $\mathfrak{H}^{\alpha}$ and homomorphisms $\pi_{\beta}^{\alpha}$ is called the direct spectrum $\left[\mathcal{H}^{\alpha} ; \pi_{\beta}^{\alpha}\right]$. The set-theoretical sum $\cup_{\alpha} \mathcal{H}^{\alpha}$ of all groups $\mathcal{H}^{\alpha}$ is called the spectral set of the given direct spectrum. Two elements of the spectral set, $h^{\alpha} \varepsilon \mathcal{F C}^{\alpha}$ and $h^{\beta} \varepsilon \mathcal{F}^{\beta}$ are called equivalent if there exists in the spectrum a group $\mathfrak{F}^{\gamma}$ such that $\mathfrak{F}^{\gamma}>\mathfrak{F}^{\alpha}, \mathfrak{F}^{\gamma}>\mathcal{F}^{\beta}$ and $\pi_{\gamma}^{\alpha} h^{\alpha}=\pi_{\gamma}^{\beta} h^{\beta}$.

This notion of equivalence obviously possesses the properties of reflextivity, symmetry and, in virtue of the unboundedness of the partially ordered set $D$, also of transitivity. The spectral set $\cup_{\alpha} \mathcal{F}^{\alpha}$ falls therefore into classes of equivalence which we shall for the sake of shortness call the bundles of the direct spectrum $\left[\mathcal{H C}^{\alpha} ; \pi_{\beta}^{\alpha}\right]$. The bundles possess the following obvious property: every projection of an element of any bundle is an element of the same bundle. Hence follows: If $h^{\alpha} \varepsilon \mathcal{F}^{\alpha}$ is an element of the bundle $h$ and $\mathfrak{F}^{\beta}>\mathfrak{H}^{\alpha}$, then in $\mathfrak{F}^{\beta}$ there is an element $h^{\beta}$ of the bundle $h$.

In any two bundles $h_{1}$ and $h_{2}$ we may find elements $h_{1}^{\alpha}$ and $h_{2}^{\alpha}$ belonging to one and the same $\mathfrak{H}^{\alpha}$. In fact, choose arbitrarily $h^{\alpha_{1}} \varepsilon h_{1}$ and $h^{\alpha_{2}} \varepsilon h_{2}$ and take $\mathfrak{H}^{\alpha}>\mathfrak{H}^{\alpha_{1}}, \mathfrak{H}^{\alpha}>\mathfrak{H}^{\alpha_{2}}$. Then

$$
\pi_{\alpha}^{\alpha_{1}} h^{\alpha_{1}}=h_{1}^{\alpha} \varepsilon h_{1}, \quad \pi_{\alpha}^{\alpha_{2}} h^{\alpha_{2}}=h_{2}^{\alpha} \varepsilon h_{2} .
$$

Let $h_{1}$ and $h_{2}$ be two bundles. From the above follows that we can find two elements $h_{1}^{\alpha} \varepsilon h_{1}, h_{2}^{\alpha} \varepsilon h_{2}$ belonging to one and the same group $\mathcal{F}^{\alpha}$. We shall call the bundle $h$, containing the element $h^{\alpha}=h_{1}^{\alpha}+h_{2}^{\alpha}$ the sum of the bundles $h_{1}$ and $h_{2}$. This definition does not depend on the choice of the elements $h_{1}^{\alpha} \varepsilon h_{1}$ and $h_{2}^{\dot{\alpha}} \varepsilon h_{2}$. In fact, if $h_{1}^{\beta} \varepsilon h_{1}, h_{2}^{\beta} \varepsilon h_{2}$, then $h^{\beta}=h_{1}^{\beta}+h_{2}^{\beta}$ belongs to the same bundle as $h^{\alpha}$. In order to prove this observe that since $h_{1}^{\alpha}$ and $h_{1}^{\beta}$ belong to one and the same bundle, there exists such an $\mathcal{F}^{\gamma_{1}}$,

$$
\mathfrak{H}^{\gamma_{1}}>\mathfrak{H}^{\alpha}, \mathfrak{H}^{\gamma_{1}}>\mathfrak{H}^{\beta},
$$

that

$$
\stackrel{\alpha}{\pi_{\gamma_{1}}} \stackrel{\alpha}{\alpha}=\stackrel{\beta}{\beta} h_{\gamma_{1}}^{\beta} h_{1}^{\beta}=h^{\gamma_{1}} .
$$

Similarly there exists an $\mathfrak{F}^{\gamma_{2}}$ such that 


$$
\underset{\pi_{\gamma_{2}}}{\alpha} h_{2}^{\alpha}=\pi_{\gamma_{2}}^{\beta} h_{2}^{\beta}=h^{\gamma_{2}} .
$$

Take an $\mathcal{F}^{\gamma}$ following after $\mathcal{F}^{\mathcal{C}_{1}}$ as well as after $\mathcal{F}^{\boldsymbol{C}_{2}}$. Then

$$
\pi_{\gamma}^{\gamma_{1}} h_{1}^{\gamma}=\pi_{\gamma}^{\alpha} h_{1}^{\alpha}=\pi_{\gamma}^{\beta} h_{1}^{\beta}=h_{1}^{\gamma} ; \quad \pi_{\gamma}^{\gamma_{2}} h_{2}^{\gamma}=\pi_{\gamma}^{\alpha} h_{2}^{\alpha}=\pi_{\gamma}^{\beta} h_{2}^{\beta}=h_{2}^{\gamma}
$$

and, consequently,

$$
h^{\gamma}=h_{1}^{\gamma}+h_{2}^{\gamma}=\pi_{\gamma}^{\alpha} h^{\alpha}=\pi_{\gamma}^{\beta} h^{\beta},
$$

so that the equivalence of $h^{\alpha}=h_{1}^{\alpha}+h_{2}^{\alpha}$ and $h^{\beta}=h_{1}^{\beta}+h_{2}^{\beta}$ is proved.

The so-defined addition of bundles is obviously associative. It turns the set of all bundles into a (discrete) group $\mathfrak{H C}=\lim _{\rightarrow}\left[\mathfrak{F}^{\alpha} ; \pi_{\beta}^{\alpha}\right]$ which is called the limit group of the direct spectrum $\left[\mathfrak{H}^{\alpha} ; \pi_{\beta}^{\alpha}\right]$. The zero elements of all groups $\mathcal{H}^{\alpha}$ are all equivalent to each other and, consequently, belong to one and the same bundle - the zero bundle, which is the zero element of the group $\mathfrak{F C}$.

The elements $-h^{\alpha}$ opposite to the elements $h^{\alpha}$ of a bundle $h$ form the bundle $-h$.

Correlating to every element $h^{\alpha}$ of the group $\mathfrak{F}^{\alpha}$ the bundle containing this element, we obtain a homomorphic mapping $\pi^{\alpha}$ of the group $\mathcal{F}^{\alpha}$ into the group $\mathcal{H}$. For $\mathfrak{H}^{\alpha}<\mathfrak{H}^{\beta}$ we have

$$
\pi^{\alpha}=\stackrel{\beta}{\beta} \underset{\alpha}{\alpha}
$$

$3: 3$. Let two spectra-the direct spectrum

$$
\left[H^{\alpha} ; \tilde{\omega}_{\alpha}^{\beta}\right]
$$

and the inverse spectrum

$$
\left[\mathfrak{H C}^{\alpha} ; \pi_{\beta}^{\sim}\right]
$$

-be given. If the groups $H^{\alpha}$ and $\mathcal{H}^{\alpha}$ composing these spectra are dual to each other for every given $\alpha$ and the homomorphisms $\tilde{\omega}_{\alpha}^{\beta}$ and $\pi_{\beta}^{\alpha}$ are conjugate, then the spectra are said to be conjugate to each other. It is known that the limit groups $H$ and $\mathfrak{H}$ of two conjugate spectra are dual (Steenrod( $\left.{ }^{4}\right)$ ) and that an element $h$ of the group $\mathfrak{H C}=\lim .\left[\mathfrak{H}^{\alpha} ; \pi_{\beta}^{\alpha}\right]$ realizes a homomorphism of the group $H=\lim _{\leftarrow}\left[H^{\alpha} ; \tilde{\omega}_{\alpha}^{\beta}\right]$ in $\kappa$ according to the formula :

$$
\eta^{h}=\eta^{\alpha} h^{\alpha}
$$

where $\eta^{\alpha}$ and $h^{\alpha}$ are taken arbitrarily in $\eta$ and $h$ : it turns out that the sodefined homomorphism does not depend on the elements of arbitrariness involved in its definition.

3.4. In the sequel we shall almost exclusively consider direct spectra; therefore by a "spectrum" without any adjective we shall understand a direct spectrum and in accordance with this omit the arrows in the formulae. 
3.41. Let two spectra

$$
\left[U^{\alpha} ; \pi_{\beta}^{\alpha}\right], \quad\left[V^{\alpha} ; \rho_{\beta}^{\alpha}\right]
$$

be given, the elements of which correspond to each other in one-to-one manner $\left(U^{\alpha} \rightleftarrows V^{\alpha}\right.$ ). For every $\alpha$ let there be given a homomorphic mapping $\phi_{\alpha}$ of the group $U^{\alpha}$ in the group $V^{\alpha}$. If for every $\alpha$, every $U^{\alpha}$, and every $u^{\alpha} \varepsilon U^{\alpha}$ the condition

$$
\stackrel{\alpha}{\rho_{\beta} \phi_{\alpha} u^{\alpha}=\phi_{\beta} \pi_{\beta}^{\alpha} u^{\alpha}}
$$

is satisfied, then we obtain a homomorphic mapping $\phi$ of the group $U=\lim \left[U^{\alpha} ; \pi_{\beta}^{\alpha}\right]$ in the group $V=\lim \left[V^{\alpha} ; \rho_{\beta}^{\alpha}\right]$ as the mapping correlating to every bundle $u$ of the spectrum $\left[U^{\alpha} ; \pi_{\beta}^{\alpha}\right]$ the bundle $v=\phi u$ of the spectrum $\left[V^{\alpha} ; \rho_{\beta}^{\alpha}\right]$ containing with an element $u^{\alpha} \varepsilon U^{\alpha}$ also the element $\phi_{\alpha} u^{\alpha}$.

It suffices to show: from $u^{\alpha} \equiv u^{\beta}$ (where $\equiv$ is the sign of equivalence) follows $\phi_{\alpha} u^{\alpha} \equiv \phi_{\beta} u^{\beta}$. But if $u^{\alpha} \equiv u^{\beta}$, then there exists such a $U^{\gamma}$ that

$$
\pi_{\gamma}^{\alpha} u^{\alpha}=\pi_{\gamma}^{\beta} u^{\beta}
$$

and, consequently,

q.e.d.

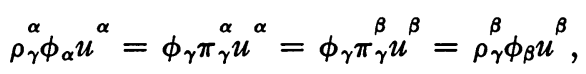

We add two remarks, the proofs of which may be left to the reader.

3.411. If, whatever be $\alpha$ and $v^{\alpha} \varepsilon V^{\alpha}$, there is $a V^{\beta}>V^{\alpha}$ and $a u^{\beta} \varepsilon U^{\beta}$ such that

$$
\phi_{\beta} u^{\beta}=\stackrel{\alpha}{\rho_{\beta} v^{\alpha}}
$$

then the mapping $\phi$ is a homomorphism of the group $U$ on the group $V$.

3.412. If from $\phi u=0$ it follows that $u \varepsilon U$ contains the zero element of some group $U^{\alpha}$, then $\phi$ is an isomorphic mapping.

3.5. A partially ordered set $D$ is called a part of the partially ordered set $D^{\prime}$, if every element of $D$ is an element of $D^{\prime}$ and if from $d_{1}>d_{2}$ in $D$ follows $d_{1}>d_{2}$ in $D^{\prime}$ (but it is not demanded that from $d_{1}>d_{2}$ in $D^{\prime}, d_{1} \varepsilon D, d_{2} \varepsilon D$, should necessarily follow $d_{1}>d_{2}$ in $D$ ).

A part $D$ of an unbounded partially ordered set $D^{\prime}$ is called cofinal to the whole $D^{\prime}$ if $D$ is unbounded and after every element of $D^{\prime}$ in $D^{\prime}$ follows an element of $D$.

A spectrum I is called a part, respectively a cofinal part, of a spectrum II, if the spectrum I considered as a partially ordered set of groups, of which it is composed, is a part, respectively a cofinal part, of the spectrum II and if the projections in I coincide with corresponding projections in II.

Let now the spectrum I form a part of the spectrum II. It is obvious that two elements of the spectral set of I equivalent in the spectrum I are equiva- 
lent also in the spectrum II. If I is a cofinal part of II, then, conversely, two elements of I equivalent in the spectrum II are equivalent also in the spectrum I. From the first assertion it follows that every bundle of the spectrum $I$ is contained in a bundle of the spectrum II. From the second assertion it follows that in the case of cofinality of I and II every bundle of the spectrum II contains only one bundle of the spectrum I (that every bundle of II contains at least one bundle of I follows directly from the condition of cofinality).

Thus we have

3.51. If one of two spectra forms a cofinal part of the other, then both spectra have isomorphic limit groups.

3.6. We shall also need the following proposition.

3.61. Suppose that the spectra

$$
\left[U^{\alpha} ; \pi_{\beta}^{\alpha}\right], \quad\left[U^{\alpha \lambda} ; \pi_{\beta \mu}^{\alpha \lambda}\right]
$$

satisfy the following conditions:

$1^{\circ}$. There exists an isomorphic mapping $\phi_{\alpha}^{\alpha \lambda}$ of the group $U^{\alpha \lambda}$ on the group $U^{\alpha}$.

$2^{\circ}$. If $U^{\beta \mu}>U^{\alpha \lambda}$, then $U^{\beta}>U^{\alpha}$.

$3^{\circ}$. For every $u^{\alpha \lambda} \varepsilon U^{\alpha \lambda}$ we have

$$
\pi_{\beta \mu}^{\alpha \lambda} u^{\alpha \lambda}=\left(\phi_{\mu}^{\beta \mu}\right)^{-1} \pi_{\beta}^{\alpha} \phi_{\alpha}^{\alpha \lambda} u^{\alpha \lambda} .
$$

Then the limit groups $\lim \left[U^{\alpha \lambda} ; \pi_{\beta \mu}^{\alpha \lambda}\right]$ and $\lim \left[U^{\alpha} ; \pi_{\beta}^{\alpha}\right]$ are isomorphic.

For the proof construct first from the groups $U^{\alpha \lambda}$ a partially ordered set $D^{\prime}$ by putting always $U^{\beta \mu}>U^{\alpha \lambda}$, if in the spectrum $\left[U^{\alpha} ; \pi_{\beta}^{\alpha}\right]$ the inequality $U^{\beta}>U^{\alpha}$ holds. The partially ordered set $D^{\prime}$ is evidently unbounded.

We define now the projections

Since

$$
\rho_{\beta \mu}^{\alpha \lambda} u^{\alpha \lambda}=\left(\phi_{\beta}^{\beta \mu}\right)^{-1} \pi_{\beta}^{\alpha} \phi_{\alpha}^{\alpha \lambda} u^{\alpha \lambda}
$$

$$
\stackrel{\beta \mu}{\beta \mu \lambda}=\left(\phi_{\gamma \nu}^{\gamma \nu}\right)^{-1} \pi_{\gamma \mu}^{\beta} \phi_{\beta}^{\beta \mu}\left(\phi_{\beta}^{\beta \mu}\right)^{-1} \underset{\pi_{\beta} \phi_{\alpha}^{\alpha \lambda}}{\rho_{\alpha}}=\left(\phi_{\gamma}^{\gamma \nu}\right)^{-1} \underset{\pi_{\gamma} \phi_{\alpha}^{\alpha \lambda}}{\alpha \lambda}=\rho_{\gamma \nu}^{\alpha \lambda}
$$

$\left[U^{\alpha \lambda} ; \rho_{\beta \mu}^{\alpha \lambda}\right]$ is obviously a spectrum containing the spectrum $\left[U^{\alpha \lambda} ; \pi_{\beta \mu}^{\alpha \lambda}\right]$ as its cofinal part. Therefore the groups $\lim \left[U^{\alpha \lambda} ; \rho_{\beta \mu}^{\alpha \lambda}\right]$ and $\lim \left[U^{\alpha \lambda} ; \pi_{\beta \mu}^{\alpha \lambda}\right]$ are isomorphic. Identifying for every $\alpha$ the group $U^{\alpha}$ with the group $U^{\alpha_{1}}$ isomorphic to it, we may also consider the spectrum $\left[U^{\alpha} ; \pi_{\beta}^{\alpha}\right]$ as a part of the spectrum $\left[U^{\alpha \lambda} ; \rho_{\beta \mu}^{\alpha \lambda}\right]$ and even as a cofinal part. In fact, in order to obtain $U^{\beta_{1}}$ following after a given $U^{\alpha \lambda}$ in the spectrum $\left[U^{\alpha \lambda} ; \rho_{\beta \mu}^{\alpha \lambda}\right]$ it is sufficient to take $U^{\beta}>U^{\alpha}$ in the spectrum $\left[U^{\alpha} ; \pi_{\beta}^{\alpha}\right]$; evidently, $U^{\beta_{1}}>U^{\alpha \lambda}$ in the spectrum $\left[U^{\alpha \lambda} ; \rho_{\beta \mu}^{\alpha \lambda}\right]$.

Thus the group $\lim \left[U^{\alpha} ; \pi_{\beta}^{\alpha}\right]$ is isomorphic to the group $\lim \left[U^{\alpha \lambda} ; \rho_{\beta \mu}^{\alpha \lambda}\right]$ and, consequently, also to the group $\lim \left[U^{\alpha \lambda} ; \pi_{\beta \mu}^{\alpha \lambda}\right]$, q.e.d. 


\section{The Formal DUALITY}

4.1. Let there be given an unbounded partially ordered set $\Omega$ of complexes $K^{\alpha}$. Suppose that in every complex $K^{\alpha}$ are given two subcomplexes $K_{0}^{\alpha}$ and $C^{\alpha}$. The complex $C^{\alpha}$ is called a special subcomplex of the complex $K^{\alpha}$. If $K^{\beta}>K^{\alpha}$, then simplicial mappings $S_{\alpha}^{\beta}$ of the complex $K^{\beta}$ into the complex $K^{\alpha}$ are defined, which are called projections of $K^{\beta}$ into $K^{\alpha}$; they satisfy the following conditions:

$1^{\circ}$. For any projection $S_{\alpha}^{\beta}$

$$
S_{\alpha}^{\beta}\left(K_{0}^{\beta}\right) \subset K_{0}^{\alpha}, \quad S_{\alpha}^{\beta}\left(C^{\beta}\right) \subset C^{\alpha} .
$$

$2^{\circ}$. If $S_{\alpha}^{\beta}$ and $\bar{S}_{\alpha}^{\beta}$ are two projections of $K^{\beta}$ into $K^{\alpha}$, then for any simplex $t_{\beta} \varepsilon K^{\beta}$ the simplexes $S_{\alpha}^{\beta} t_{\beta}$ and $\bar{S}_{\alpha}^{\beta} t_{\beta}$ are faces of a certain simplex $t_{\alpha} \varepsilon K^{\alpha}$ and, if $t_{\beta} \varepsilon K_{0}^{\beta}$ or $t_{\beta} \varepsilon C^{\beta}$, then we may assume that correspondingly $t_{\alpha} \varepsilon K_{0}^{\alpha}, t_{\alpha} \varepsilon C^{\alpha}$.

From the condition $1^{\circ}$ it follows that every projection $S_{\alpha}^{\beta}$ generates a homomorphism $\sigma_{\beta}^{\alpha}$ of the groups

$$
L^{r}\left(K^{\alpha}-C^{\alpha}\right), \quad L^{r}\left(K_{0}^{\alpha}-C^{\alpha}\right), \quad L^{r}\left(K^{\alpha}-K_{0}^{\alpha}-C^{\alpha}\right)\left({ }^{8}\right)
$$

respectively into the groups

$$
L^{r}\left(K^{\beta}-C^{\beta}\right), \quad L^{r}\left(K_{0}^{\beta}-C^{\beta}\right), \quad L^{r}\left(K^{\beta}-K_{0}^{\beta}-C^{\beta}\right) ;
$$

the homomorphisms $\sigma_{\beta}^{\alpha}$ generate further homomorphical mappings $\pi_{\beta}^{\alpha}$ of the groups

$$
B_{\nabla}^{r}\left(K_{0}^{\alpha}-C^{\alpha}\right), \quad B_{\nabla}^{r}\left(K^{\alpha}-K_{0}^{\alpha}-C^{\alpha}\right)
$$

in to the groups

$$
B_{\nabla}^{r}\left(K_{0}^{\beta}-C^{\beta}\right), \quad B_{\nabla}^{r}\left(K^{\beta}-K_{0}^{\beta}-C^{\beta}\right)
$$

and from the condition $2^{\circ}$ it follows that all projections of the complex $K^{\beta}$ into the complex $K^{\alpha}$ generate one and the same homomorphism $\pi_{\beta}^{\alpha}$ of the groups (4.11) into the groups (4.12). These homomorphisms are also called projections.

Suppose that beside the conditions $1^{\circ}$ and $2^{\circ}$ the following condition is also satisfied:

$3^{\circ}$. If $K^{\gamma}>K^{\beta}>K^{\alpha}$, then whatever be the projections $S_{\alpha}^{\beta}$ and $S_{\beta}^{\gamma}$ of respectively $K^{\beta}$ into $K^{\alpha}$ and $K^{\gamma}$ into $K^{\beta}$, the simplicial mapping $S_{\alpha}^{\beta} S_{\beta}^{\gamma}$ of the complex $K^{\gamma}$ into the complex $K^{\alpha}$ is a projection.

From the condition $3^{\circ}$ it follows that

$$
\underset{\pi_{\gamma} \pi_{\beta}^{\alpha}}{\alpha}=\underset{\pi_{\gamma}^{\alpha}}{\alpha}
$$

and that we have the spectra

${ }^{(8)}$ We recall that $K_{0}^{\alpha}-C^{\alpha}$ means the set of all simplexes of $K_{0}^{\alpha}$ which do not belong to $C$, i.e., $K_{0}^{\alpha}-C^{\alpha}=K_{0}^{\alpha}-K_{0}^{\alpha} \cap C^{\alpha}$. 


$$
\begin{gathered}
{\left[B_{\nabla}^{r}\left(K^{\alpha}-C^{\alpha}\right) ; \pi_{\beta}^{\alpha}\right], \quad\left[B_{\nabla}^{r}\left(K_{0}^{\alpha}-C^{\alpha}\right) ; \pi_{\beta}^{\alpha}\right],} \\
{\left[B_{\nabla}^{r}\left(K^{\alpha}-K_{0}^{\alpha}-C^{\alpha}\right) ; \pi_{\beta}^{\alpha}\right] .}
\end{gathered}
$$

Definition 4.10. A partially ordered set $\Omega$ of complexes $K^{\alpha}$ satisfying the conditions $1^{\circ}, 2^{\circ}$ and $3^{\circ}$ is called simply-connected with respect to the dimensionality $r$ for given special subcomplexes $C^{\alpha}$, if the group

$$
\lim \left[B_{\nabla}^{r}\left(K^{\alpha}-C^{\alpha}\right) ; \pi_{\beta}^{\alpha}\right]
$$

consists only of the zero element.

The aim of the present paragraph is the proof of the following

THEOREM 4.1. If $\Omega$ is simply connected with respect to the dimensionalities $r$ and $r+1$, then the groups

$$
\lim \left[B_{\nabla}^{r}\left(K_{0}^{\alpha}-C^{\alpha}\right) ; \pi_{\beta}^{\alpha}\right], \quad \lim \left[B_{\nabla}^{r+1}\left(K^{\alpha}-K_{0}^{\alpha}-C^{\alpha}\right) ; \pi_{\beta}^{\alpha}\right]
$$

are isomorphic.

4.2. Preliminary remarks to the proof of Theorem 4.1. For the sake of shortness we shall write

$L_{\alpha}^{r}$ instead of $L^{r}\left(K^{\alpha}-C^{\alpha}\right)$,

$L_{0 \alpha}^{r}$ instead of $L^{r}\left(K_{0}^{\alpha}-C^{\alpha}\right)$,

$L_{0 \alpha}^{r}$ instead of $L^{r}\left(K^{\alpha}-K_{0}^{\alpha}-C^{\alpha}\right)$,

$Z_{0 \alpha}^{r}$ instead of $Z_{\nabla}^{r}\left(K_{0}^{\alpha}-C^{\alpha}\right)$,

$Z_{o \alpha}^{r}$ instead of $Z_{\nabla}^{r}\left(K^{\alpha}-K_{0}^{\alpha}-C^{\alpha}\right)$,

$H_{0 \alpha}^{r}$ instead of $H_{\nabla}^{r}\left(K_{0}^{\alpha}-C^{\alpha}\right)$,

$H_{o \alpha}^{r}$ instead of $H_{\nabla}^{r}\left(K^{\alpha}-K_{0}^{\alpha}-C^{\alpha}\right)$,

$B_{0 \alpha}^{r}$ instead of $B_{\nabla}^{r}\left(K_{0}^{\alpha}-C^{\alpha}\right)$,

$B_{\rho_{\alpha}}^{r}$ instead of $B_{\nabla}^{r}\left(K^{\alpha}-K_{0}^{\alpha}-C^{\alpha}\right)$,

$B_{0}^{r}$ instead of $\lim \left[B_{\nabla}^{r}\left(K_{0}^{\alpha}-C^{\alpha}\right) ; \pi_{\beta}^{\alpha}\right]$,

$B_{o}^{r}$ instead of $\lim \left[B_{\nabla}^{r}\left(K^{\alpha}-K_{0}^{\alpha}-C^{\alpha}\right) ; \pi_{\beta}^{\alpha}\right]$.

The elements of the groups

$$
B_{0 \alpha}^{r}, \quad B_{0}^{r}, \quad B_{g \alpha}^{r+1}, \quad B_{g}^{r+1}
$$

we shall denote respectively by

$$
u_{\alpha}^{r}, \quad u^{r}, \quad v_{\alpha}^{r+1}, \quad v^{r+1} .
$$

The elements of the groups

$$
L_{\alpha}^{r}, \quad L_{0 \alpha}^{r}, \quad L_{g \alpha}^{r},
$$

we shall denote respectively by

$$
f_{\alpha}^{r}, \quad f_{0 \alpha}^{r}, \quad g_{\alpha}^{r} .
$$


If some function $f_{\alpha}^{r}$ is given, we denote by $\bar{f}_{\alpha}^{r}$ the function which is equal to $f_{\alpha}^{r}$ on $K_{0}^{\alpha}$ and is equal to zero on $K^{\alpha}-K_{0}^{\alpha}$; by $A f_{\alpha}^{r}$ we denote the function $f_{0 \alpha}^{r}$ equal to $f_{\alpha}^{\tau}$ on $K_{0}^{\alpha}$.

If a function $f_{0 \alpha}^{r}$ is given, we denote by $E f_{0 \alpha}^{r}$ the function $f_{\alpha}^{\tau}$ equal to $f_{0 \alpha}^{r}$ on $K_{0}^{\alpha}$ and to zero on $K^{\alpha}-K_{0}^{\alpha}$.

Let us now formulate some simple properties of the operators $A$ and $E$ :

$$
\begin{gathered}
E A f_{\alpha}^{r}=\bar{f}_{\alpha}^{r}, \\
A E f_{0 \alpha}^{r}=f_{0 \alpha}^{r} .
\end{gathered}
$$

From

$$
g_{\alpha}^{r} \varepsilon L_{g \alpha}^{r}
$$

follows

$$
A_{g \alpha}^{r}=0 .
$$

If $t_{0 \alpha}^{r+1} \varepsilon K_{0}^{\alpha}$, then

$$
\begin{aligned}
\nabla A f_{\alpha}^{r}\left(t_{0 \alpha}^{r+1}\right) & =\nabla f_{\alpha}^{r}\left(t_{0 \alpha}^{r+1}\right), \\
\nabla E f_{0 \alpha}^{r}\left(t_{0 \alpha}^{r+1}\right) & =\nabla f_{0 \alpha}^{r}\left(t_{0 \alpha}^{r+1}\right) .
\end{aligned}
$$

Further,

$$
\nabla A f_{\alpha}^{r}=A \nabla f_{\alpha}^{r}
$$

In fact, if $t_{0 \alpha}^{r+1} \varepsilon K_{0}^{\alpha}$, then $\nabla A f_{\alpha}^{r}\left(t_{0 \alpha}^{r+1}\right)$ and $A \nabla f_{\alpha}^{\tau}\left(t_{0 \alpha}^{r+1}\right)$ coincide with $\nabla f_{\alpha}^{r}\left(t_{0 \alpha}^{r+1}\right)$. If $S_{\alpha}^{\beta}$ is any projection of $K^{\beta}$ into $K^{\alpha}$, then

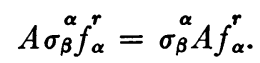

In fact, from $t_{0 \beta}^{r} \varepsilon K_{0}^{\beta}$ follows

$$
A \stackrel{\alpha}{\alpha} f_{\alpha}^{r}\left(t_{0 \beta}^{r}\right)=\stackrel{\alpha}{\alpha} f_{\alpha}^{r}\left(t_{0 \beta}^{r}\right)=f_{\alpha}^{r}\left(S_{\alpha}^{\beta} t_{0 \beta}^{r}\right), \quad \underset{\sigma_{\beta} A}{\alpha} f_{\alpha}^{r}\left(t_{0 \beta}^{r}\right)=A f_{\alpha}^{r}\left(S_{\alpha}^{\beta} t_{0 \beta}^{r}\right)=f_{\alpha}^{r}\left(S_{\alpha}^{\beta} t_{0 \beta}^{r}\right) .
$$

Observe, finally, that from $f_{0 \alpha}^{r} \varepsilon Z_{0 \alpha}^{r}$ follows $\nabla E f_{0 \alpha}^{r} \varepsilon Z_{0 \alpha}^{r+1}$. In fact, for $t_{\alpha}^{r+1} \varepsilon C^{\alpha}$ we have

$$
\nabla E f_{0 \alpha}^{r}\left(t_{\alpha}^{r+1}\right)=0,
$$

since $E f_{0 \alpha}^{r} \varepsilon L_{\alpha}^{r}$ and, consequently, $\nabla E f_{0 \alpha}^{r} \varepsilon L_{\alpha}^{r+1}$, and for $t_{\alpha}^{r+1} \varepsilon K_{0}^{\alpha}$,

$$
\nabla E f_{0 \alpha}^{r}\left(t_{\alpha}^{r+1}\right)=\nabla f_{0 \alpha}^{r}\left(t_{\alpha}^{r+1}\right)=0,
$$

since $f_{0 \alpha}^{r} \varepsilon Z_{0 \alpha}^{r}$.

4.3. We proceed now to prove Theorem 4.1.

To each $f_{0 \alpha}^{r} \varepsilon Z_{0 \alpha}^{r}$ corresponds a definite $g_{\alpha}^{r+1} \varepsilon Z_{o \alpha}^{r+1}$, namely $\nabla E f_{0 \alpha}^{r}$. 
4.31. The operator $\nabla E$, applied to the group $Z_{0 \alpha}^{r}$, generates an homomorphism $\phi_{\alpha}$ of the group $B_{0 \alpha}^{r}$ into the group $B_{g \alpha}^{r+1}$.

In order to prove this it is sufficient to show that if $f_{0 \alpha}^{r} \varepsilon H_{0 \alpha}^{r}$ then $\nabla E_{0 \alpha}^{r} \varepsilon H_{o \alpha}^{r+1}$.

Let $f_{0 \alpha}^{r} \varepsilon H_{0 \alpha}^{r} ;$ then

$$
f_{0 \alpha}^{r}=\nabla f_{0 \alpha}^{r-1}
$$

Put

$$
f_{\alpha}^{r-1}=E f_{0 \alpha}^{r-1}
$$

For $t_{0 \alpha}^{r} \varepsilon K_{0}^{\alpha}$ we have

$$
f_{0 \alpha}^{r}\left(t_{0 \alpha}^{r}\right)=\Delta f_{\alpha}^{r-1}\left(t_{0 \alpha}^{r}\right) .
$$

Put

$$
\begin{aligned}
& h_{\alpha}^{r}\left(t_{\alpha}^{r}\right)=0, \text { if } t_{\alpha}^{r} \varepsilon K_{0}^{\alpha}, \\
& h_{\alpha}^{r}\left(t_{\alpha}^{r}\right)=\nabla f_{\alpha}^{r-1}\left(t_{\alpha}^{r}\right), \text { if } t_{\alpha}^{r} \varepsilon K^{\alpha}-K_{0 .}^{\alpha}
\end{aligned}
$$

From (4.32), (4.33), (4.34) follows

$$
h_{\alpha}^{r}\left(t_{\alpha}^{r}\right)+E f_{0 \alpha}^{r}\left(t_{\alpha}^{r}\right)=\nabla f_{\alpha}^{r-1}\left(t_{\alpha}^{r}\right)
$$

for any $t_{\alpha}^{\tau} \varepsilon K^{\alpha}$, whence

i.e.,

$$
\nabla h_{\alpha}^{r}+\nabla E f_{0 \alpha}^{r}=0, \quad \nabla E f_{0 \alpha}^{r}=-\nabla h_{\alpha}^{r},
$$

$$
\nabla E f_{0 \alpha}^{r} \varepsilon H_{o \alpha}^{r+1} .
$$

Let us now show that for any $u_{\alpha}^{r} \varepsilon B_{0 \alpha}^{r}$

$$
\underset{\pi_{\beta} \phi_{\alpha}}{\alpha} u_{\alpha}^{r}=\phi_{\beta} \pi_{\beta}^{\alpha} u_{\alpha}^{r} .
$$

Let there be chosen some projection $S_{\alpha}^{\beta}$ of the complex $K_{\alpha}^{\beta}$ into the complex $K^{\alpha}$. Choose arbitrarily $f_{0 \alpha}^{r} \varepsilon u_{\alpha}^{r}$. In order to prove the formula (4.35) it is sufficient to show that under the homomorphism $\sigma_{0 \beta}^{0 \alpha}$ of the group $Z_{0 \alpha}^{r}$ into the group $Z_{0 \beta}^{r}$ generated by the projection $S_{\alpha}^{\beta}$ we have

$$
\underset{\sigma_{\beta} \nabla E f_{0 \alpha}^{r}}{\alpha} \sim \nabla E \sigma_{\beta}^{\alpha} f_{0 \alpha}^{r} \text { in } K^{\beta}-K_{0}^{\beta}-C^{\beta} .
$$

Having in view that

$$
\stackrel{\alpha}{\sigma_{\beta} \nabla}=\nabla \stackrel{\alpha}{\alpha},
$$

we may write (4.351) in the form

$$
\nabla \underset{\beta}{\alpha} E f_{0 \alpha}^{r}-\nabla E \sigma_{\beta}^{\alpha} f_{0 \alpha}^{r} \sim 0 \quad \text { in } K^{\beta}-K_{0}^{\beta}-C^{\beta} .
$$


But (4.352) obviously follows from

$$
\underset{\sigma_{\beta}}{\alpha} E f_{0 \alpha}^{r}-E \sigma_{\beta}^{\alpha} f_{0 \alpha}^{r} \varepsilon L_{g \beta}^{r} .
$$

We have thus only to verify this last formula. But

that is,

$$
\sigma_{\beta}^{\alpha} E f_{0 \alpha}^{r}\left(t_{\beta}^{r}\right)=E f_{0 \alpha}^{r}\left(S_{\alpha}^{\beta} t_{\beta}^{r}\right)
$$

$$
\begin{array}{rlrl}
\underset{\sigma_{\beta}^{\alpha} E f_{0 \alpha}^{r}\left(t_{\beta}^{r}\right)}{r} & =0, & & \text { if } S_{\alpha}^{\beta} t_{\beta}^{r} \varepsilon C^{\alpha}, \\
& =f_{0 \alpha}^{r}\left(S_{\alpha}^{\beta} t_{\beta}^{r}\right), & \text { if } S_{\alpha}^{\beta} t_{\beta}^{r} \varepsilon K_{0}^{\alpha}, \\
& =0, & & \text { if } S_{\alpha}^{\beta} t_{\beta}^{r} \varepsilon K^{\alpha}-K_{0 .}^{\alpha} .
\end{array}
$$

On the other hand,

$$
\begin{aligned}
& E \sigma_{\beta}^{\alpha} f_{0 \alpha}^{r}\left(t_{\beta}^{r}\right)=0, \quad t_{\beta}^{r} \varepsilon C^{\beta}, \\
& E \sigma_{\beta}^{\alpha} f_{0 \alpha}^{r}\left(t_{\beta}^{r}\right)=\sigma_{\beta}^{\alpha} f_{0 \alpha}^{r}\left(t_{\beta}^{r}\right)=f_{0 \alpha}^{r}\left(S_{\alpha}^{\beta} t_{\beta}^{r}\right), \text { if } t_{\beta}^{r} \varepsilon K_{0}^{\beta}, \\
& E \sigma_{\beta}^{\alpha} f_{0 \alpha}^{r}\left(t_{\beta}^{r}\right)=0, \text { if } t_{\beta}^{r} \varepsilon K^{\beta}-K_{0}^{\beta} .
\end{aligned}
$$

If $t_{\beta}^{\tau} \varepsilon K_{0}^{\beta}$ or $t_{\beta}^{\tau} \varepsilon C^{\beta}$, then, respectively,

$$
S_{\alpha}^{\beta} t_{\beta}^{r} \varepsilon K_{0}^{\alpha}, \quad S_{\alpha}^{\beta} t_{\beta}^{r} \varepsilon C^{\alpha} .
$$

Therefore for

we have

$$
\stackrel{r}{t_{\beta} \varepsilon K_{0}^{\beta},} \quad \stackrel{r}{t_{\beta} \varepsilon C^{\beta},}
$$

$$
\sigma_{\beta}^{\alpha} E f_{0 \alpha}^{r}\left(t_{\beta}^{r}\right)=E \sigma_{\beta}^{\alpha} f_{0 \alpha}^{r}\left(t_{\beta}^{r}\right),
$$

whence follows (4.353) and so (4.352), (4.351) and (4.35).

From (4.35) and (3.41) follows

4.32. The operator $\nabla E$ determines through the homomorphisms $\phi_{\alpha}$ the homomorphism $\phi$ of the group $B_{0}^{r}$ into the group $B_{0}^{r+1}$.

If $u^{r}$ is an element of the group $B_{0}^{r}$, then take any element $u_{\alpha}^{r}$ of the bundle $u^{r}$ and any cycle $f_{\alpha}^{r}$ contained in the homologic class of $u^{r}$. The element $u_{\alpha}^{r+1}$ of the group $B_{\rho \alpha}^{r+1}$ containing the cycle $\nabla E f_{\alpha}^{r}$ is contained in the bundle $v^{r+1}$, which is by definition the element $\phi\left(u^{r}\right)$ of the group $B_{g}^{r+1}$.

4.4. The homomorphism $\phi$ is a mapping of the group $B_{0}^{r}$ on the group $B_{\theta}^{r+1}$. In order to prove this it is, on ground of (3.411), sufficient to show that

4.41. Whatever be $\alpha$ and $v_{\alpha}^{r+1} \varepsilon B_{g \alpha}^{r+1}$, there is always $a K^{\beta}>K^{\alpha}$ and $a u_{\beta}^{r} \varepsilon B_{0 \beta}^{r}$ such that

$$
\phi_{\beta} u_{\beta}^{r}=\underset{\pi_{\beta} v_{\alpha}^{r+1}}{\stackrel{\alpha}{r}}
$$


Let $v_{\alpha}^{r+1}$ be given. Take a $g_{\alpha}^{r+1} \varepsilon v_{\alpha}^{r+1}$. Since $K$ is simply connected with respect to the dimensionality $r+1$, there is always a $K^{\beta}>K^{\alpha}$ such that

$$
\underset{\sigma_{\beta}}{\alpha} g_{\alpha}^{r+1} \sim 0
$$

Thus there exists such an $f_{\beta}^{r}$ that

$$
\nabla f_{\beta}^{r}=\underset{\sigma_{\beta}}{\alpha} g_{\alpha}^{r+1}
$$

Putting

$$
\stackrel{\alpha}{\sigma_{\beta} g_{\alpha}^{r+1}}=g_{\beta}^{r+1}
$$

we have

$$
g_{\beta}^{r+1} \varepsilon v_{\beta}^{r+1} \varepsilon \pi_{\beta}^{\alpha} v_{\alpha}^{r+1}
$$

If $t_{\beta}^{r+1} \varepsilon K_{0}^{\beta}$, then

$$
\nabla A f_{\beta}^{r}\left(t_{\beta}^{r+1}\right)=\nabla f_{\beta}^{r}\left(t_{\beta}^{r+1}\right)=0,
$$

i.e.,

$$
A f_{\beta}^{r} \varepsilon Z_{0 \beta}^{r} .
$$

Observing that always $E A f_{\beta}^{r}=\bar{f}_{\beta}^{r}$, put

$$
g_{\beta}^{r}=f_{\beta}^{r}-E A f^{r}=f_{\beta}^{r}-\bar{f}_{\beta}^{r} \varepsilon L_{o \beta}^{r} .
$$

Evidently

$$
\nabla g_{\beta}^{r}=\nabla f_{\beta}^{r}-\nabla E A f_{\beta}^{r} .
$$

Consequently, in view of (4.41) and (4.42), .

$$
g_{\beta}^{r+1} \sim \Delta E A f_{\beta}^{r} \quad \text { in } K^{\beta}-K_{0}^{\beta}-C^{\beta},
$$

i.e. (on ground of (4.43)),

$$
\nabla E A f_{\beta}^{r} \varepsilon \underset{\pi_{\beta} v_{\alpha}^{r+1}}{\alpha}
$$

If $A f_{\beta}^{r} \varepsilon u_{\beta}^{r} \varepsilon B_{\beta}^{r}$, then

$$
\phi_{\beta} u_{\beta}^{r}=\underset{\pi_{\beta} v_{\alpha}^{r+1}}{\stackrel{\alpha}{*},}
$$

and 4.41 is proved.

4.5. The homomorphism $\phi$ is an isomorphism.

LEMMA 4.51. If $f_{\alpha}^{\tau} \varepsilon Z_{\nabla}^{\tau}\left(K^{\alpha}\right)$, then there exists such $a K^{\beta}>K^{\alpha}$ that

$$
A \sigma_{\beta}^{\alpha} f_{\alpha}^{r} \sim 0 \quad \text { in } K_{0}^{\beta}-C^{\beta} .
$$


In fact, since $K$ is simply-connected with respect to the dimensionality $r$, in some $K^{\beta}>K^{\alpha}$ there exists such an $f_{\beta}^{r-1} \subset K^{\beta}-C^{\beta}$ that

$$
\sigma_{\beta}^{\alpha} f_{\alpha}^{r}=\nabla f_{\beta}^{r-1}
$$

Hence

$$
A \sigma_{\beta}^{\alpha} f_{\alpha}^{r}=A \nabla f_{\beta}^{r-1}=\nabla A f_{\beta}^{r-1},
$$

and Lemma 4.51 is proved.

For the proof of the assertion 4.5 it is sufficient, in virtue of $(3.412)$, to show that if

$$
\phi u^{r}=0
$$

and

$$
f_{0 \alpha}^{r} \varepsilon u_{\alpha}^{r} \varepsilon u^{r}
$$

then there exists such a $K^{\beta}>K^{\alpha}$ that

$$
\stackrel{\alpha}{\sigma_{\beta}^{\gamma} f_{0 \alpha}^{r}} \sim 0 \quad \text { in } K_{0}^{\beta}-C^{\beta} .
$$

From (4.52) it follows that (4.53) may be from the outset chosen in such a way that

$$
\nabla E f_{0 \alpha}^{r}=\nabla g_{\alpha}^{r}, \quad g_{\alpha}^{r} \varepsilon L_{o \alpha}^{r} .
$$

Evidently $E f_{0 \alpha}^{r}-g_{\alpha}^{r}$ is a cycle and hence, in virtue of Lemma 4.51, we may choose $K^{\beta}>K^{\alpha}$ such that

$$
A\left(\sigma_{\beta}^{\alpha} E f_{0 \alpha}^{r}-\sigma_{\beta}^{\alpha} g_{\alpha}^{r}\right) \sim 0 \quad \text { in } K_{0}^{\beta}-C^{\beta} .
$$

Since

$$
\begin{aligned}
A \sigma_{\beta}^{\alpha} g_{\alpha}^{r} & =0, \\
A \sigma_{\beta}^{\alpha} E f_{0 \alpha}^{r} & \sim 0
\end{aligned}
$$$$
\text { in } K_{0}^{\beta}-C^{\beta} \text {, }
$$

and since

$$
\begin{aligned}
& A \sigma_{\beta}^{\alpha} E f_{0 \alpha}^{r}=\sigma_{\beta}^{\alpha} A E f_{0 \alpha}^{r}=\sigma_{\beta}^{\alpha} f_{0 \alpha}^{r}, \\
& \stackrel{\alpha}{\sigma_{\beta} f_{0 \alpha}^{r}} \sim 0
\end{aligned}
$$$$
\text { in } K_{0}^{\beta}-C^{\beta} \text {, }
$$

so that the assertion 4.5 and with it the whole Theorem 4.41 is completely proved.

4.6. Consider separately the case $r=0$. Suppose that all $K^{\alpha}$ are connected complexes, so that the groups $B_{\nabla}^{00}\left(K^{\alpha}\right)$ consist only of the corresponding zero elements.

Suppose, further, that all $C^{\alpha}=0$ and that our system of complexes $\Omega$ is simply-connected with respect to the dimensionality 1 . 
Instead of $B_{\nabla}^{00}\left(K_{0}^{\alpha}\right)$ we shall write $B_{0 \alpha}^{00}$ and instead of $\lim \left[B_{0 \alpha}^{00} ; \pi_{\beta}^{\alpha}\right]$ we shall write simply $B^{00}$.

The operator $\nabla E$ again correlates to every element $f_{0 \alpha}^{r} \varepsilon Z_{0 \alpha}^{0}$ the element

$$
g_{\alpha}^{1}=\nabla E f_{0 \alpha}^{r} \varepsilon Z_{g}^{1} .
$$

Moreover, if $f_{0 \alpha}^{0} \varepsilon Z_{0 \alpha}^{00}$, then $\nabla E f_{0 \alpha}^{0} \varepsilon H_{g \alpha}^{1}$. In fact, putting the function $f_{\alpha}^{0}$ on the whole $K^{\alpha}$ equal to the constant value of the function $f_{0 \alpha}^{0}$ on $K_{0}^{\alpha}$, we obtain on ground of 1.81 a cycle $f_{\alpha}^{0}$. Here

$$
f_{\alpha}^{0}=E f_{0 \alpha}^{0}+h_{\alpha}^{0}
$$

where

$$
h_{\alpha}^{0}=f_{\alpha}^{0} \text { on } K^{\alpha}-K_{0}^{\alpha}, \quad h_{\alpha}^{0}=0 \text { on } K_{0}^{\alpha} .
$$

Since $f_{\alpha}^{0}$ is a cycle,

i.e.,

$$
0=\nabla f_{\alpha}^{0}=\nabla E f_{0 \alpha}^{0}+\nabla h_{\alpha}^{0}
$$

$$
\nabla E f_{0 \alpha}^{0} \varepsilon H_{g \alpha}^{0} .
$$

Thus the operator $\nabla E$ applied to the group $Z_{0 \alpha}^{0}$ generates a homomorphism $\phi_{\alpha}$ of the group $B_{0 \alpha}^{00}$ into the group $B_{o \alpha}^{1}$.

Similarly, as in 4.3 we prove the formula (4.351), where now $r=0$ and the arguments are only simplified by the fact that $C^{\alpha}=0$; from (4.351) follows (4.35), where $r=0$ and $u_{\alpha}^{r}$ denotes an arbitrary element of the group $B_{0 \alpha}^{00}$. Thus the operator $\nabla E$ determines through the homomorphisms $\phi_{\alpha}$ a homomorphism $\phi$ of the group $B_{0}^{00}$ into the group $B_{0}^{1}$.

The reasonings of 4.4 remain in force and prove that the homomorphism $\phi$ is a mapping of the group $B_{0}^{00}$ on the group $B_{0}^{1}$.

Let us finally prove that $\phi$ is an isomorphism. From the connectivity of $K^{\alpha}$ and from 1.812 follows in the first place:

LEMMA. If $f_{\alpha}^{0}$ is a cycle, then

$$
A f_{\alpha} \varepsilon Z_{\alpha}^{00}
$$

In order to prove that $\phi$ is an isomorphism, it is sufficient to prove that if $u^{0} \varepsilon B_{0}^{00}$ and, further,

$$
\begin{gathered}
\phi\left(u_{0}\right)=0 \\
f_{0 \alpha} \varepsilon u_{\alpha}^{0} \varepsilon u^{0}
\end{gathered}
$$

then

$$
f_{0 \alpha}^{0} \varepsilon Z_{0 \alpha}^{00} .
$$

From (4.61) it follows that (4.62) may be from the outset so chosen that 


$$
\nabla E f_{0 \alpha}^{0}=\nabla g_{\alpha}^{0} ; \quad g_{\alpha}^{0} \varepsilon L^{0}\left(K^{\alpha}-K_{0}^{\alpha}\right) .
$$

Evidently $E f_{0 \alpha}^{0}-g_{\alpha}^{0}$ is a cycle and hence, according to the above lemma,

$$
A\left(E f_{0 \alpha}^{0}-g_{\alpha}^{0}\right) \varepsilon Z_{0 \alpha}^{00},
$$

i.e.,

$$
A E f_{0 \alpha}^{0} \varepsilon Z_{0 \alpha}^{00}
$$

i.e.,

$$
f_{0 \alpha}^{0} \varepsilon Z_{0 \alpha}^{00}
$$

Thus we have proved

THEOREM 4.6. If there is given an unbounded partially ordered set of complexes $K^{\alpha}$ connected and simply-connected with respect to the dimensionality 1 and their subcomplexes $K^{\alpha}$ satisfying the conditions of 4.1 for $C^{\alpha}=0$, then the groups

$$
\lim \left[B_{\nabla}^{00}\left(K_{0}^{\alpha}\right) ; \pi_{\beta}^{\alpha}\right], \quad \lim \left[B_{\nabla}^{1}\left(K^{\alpha}-K_{0}^{\alpha}\right) ; \pi_{\beta}^{\alpha}\right]
$$

are isomorphic.

4.7. From Theorem 4.1 follows

4.71. If $C^{\alpha}$ and $K_{0}^{\alpha}$ have no common elements and each of the groups

$$
\begin{aligned}
\lim \left[B_{\nabla}^{r}\left(K^{\alpha}-C^{\alpha}\right) ; \pi_{\beta}^{\alpha}\right], & \lim \left[B_{\nabla}^{r+1}\left(K^{\alpha}-C^{\alpha}\right) ; \pi_{\beta}^{\alpha}\right], \\
\lim \left[B_{\nabla}^{r}\left(K^{\alpha}\right) ; \pi_{\beta}^{\alpha}\right], & \lim \left[B_{\nabla}^{r+1}\left(K^{\alpha}\right) ; \pi_{\beta}^{\alpha}\right]
\end{aligned}
$$

contains only the zero element, then the groups

$$
B_{g}^{r+1}=\lim \left[B^{r+1}\left(K^{\alpha}-K_{0}^{\alpha}-C^{\alpha}\right) ; \pi_{\alpha}^{\beta}\right]
$$

and

$$
\mathfrak{B}_{g}^{r+1}=\lim \left[B_{\nabla}^{r+1}\left(K^{\alpha}-K_{0}^{\alpha}\right) ; \pi_{\beta}^{\alpha}\right]
$$

are isomorphic.

In fact, in our case $K_{0}^{\alpha}-C^{\alpha}=K_{0}^{\alpha}$ and both groups $B_{0}^{r+1}$ and $\mathfrak{B}_{0}^{r+1}$ are isomorphic to the group

$$
\lim \left[B_{\nabla}^{r}\left(K_{0}^{\alpha}\right) ; \pi_{\beta}^{\alpha}\right] .
$$

4.8. Remark. In what follows we often will have to do with unbounded partially ordered sets $\Omega$ of complexes $K^{\alpha}$ with given subcomplexes $C^{\alpha} \subset K^{\alpha}$, and of simplicial mappings $S_{\alpha}^{\beta}$ of $K^{\beta}$ into $K^{\alpha}$ ("projections"), defined for every $K^{\beta}>K^{\alpha}$. These projections will satisfy the conditions $1^{\circ}$ and $2^{\circ}$ of $\S 4.1$, with 
respect to $C^{\alpha}$ and the condition $3^{\circ}$, so that there is a direct spectrum

$$
\left[B_{\nabla}^{r}\left(\dot{K}^{\alpha}-C^{\alpha}\right) ; \pi_{\beta}^{\alpha}\right] \text {, }
$$

$\pi_{\beta}^{\alpha}$ being the homomorphism of $B_{\nabla}^{r}\left(K^{\alpha}-C^{\alpha}\right)$ into $B_{\nabla}^{r}\left(K^{\beta}-C^{\beta}\right)$, generated by $S_{\alpha}^{\beta}$. Under these circumstances the limit-group

$$
B_{\nabla}^{r}=\lim \left[B_{\nabla}^{r}\left(K^{\alpha}-C^{\alpha}\right) ; \pi_{\beta}^{\alpha}\right]
$$

can be defined in a particularly simple way. In fact, we shall consider the set $Z^{r}$ of elements of all groups $Z_{\nabla}^{r}\left(K^{\alpha}-C^{\alpha}\right)$, i.e.,

$$
Z^{r}=\cup_{\alpha} Z^{r}\left(K^{\alpha}-C^{\alpha}\right)
$$

and call $z_{\alpha}^{r} \varepsilon Z^{r}\left(K^{\alpha}-C^{\alpha}\right)$ and $z_{\beta}^{r} \varepsilon Z^{r}\left(K^{\beta}-C^{\beta}\right)$ equivalent if a $K^{\gamma}$,

$$
K^{\gamma}>K^{\alpha}, \quad K^{\gamma}>K^{\beta}
$$

and projections $S_{\alpha}^{\gamma}, S_{\beta}^{\gamma}$ of respectively $K^{\gamma}$ and $K^{\beta}$ into $K^{\alpha}$ can be found in such a way that

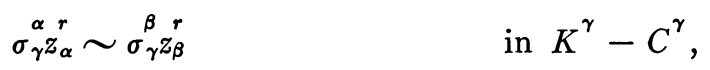

$\sigma_{\gamma}^{\alpha}, \sigma_{\gamma}^{\beta}$ being the homomorphisms of $Z^{r}\left(K^{\alpha}-C^{\alpha}\right)$ and $Z^{r}\left(K^{\beta}-C^{\beta}\right)$ in $Z^{r}\left(K^{\gamma}-C^{\gamma}\right)$ generated by $S_{\alpha}^{\gamma}$ and $S_{\beta}^{\gamma}$ respectively. Thus $Z^{r}$ is divided into classes or bundles of equivalent cycles and these bundles form a group, which, by definition, is the group $B_{\nabla}^{r}$; the addition in $B_{\nabla}^{r}$ is defined in the following way: $\zeta_{1}^{r}$ and $\zeta_{2}^{r}$ being two bundles, we take an $z_{\alpha}^{r} \varepsilon \zeta_{1}^{r}$ and a $z_{\alpha}^{\prime r} \varepsilon \zeta_{2}^{r}$ and call $\zeta_{1}^{r}+\zeta_{2}^{r}$ the bundle containing $z_{\alpha}^{r}+z_{\alpha}^{\prime r}$. It is an easy task to show that this definition of

$$
\lim \left[B_{\nabla}^{r}\left(K^{\alpha}-C^{\alpha}\right) ; \pi_{\beta}^{\alpha}\right]
$$

agees with the definition given in $\$ 3.2$.

\section{Coverings}

5.1. In the present paragraph $R$ denotes one and the same infinite set.

An indexed subset $e_{i}$ is by definition a pair consisting of a certain subset $\left|e_{i}\right|$ of the set $R$ and a natural number $i$. Two indexed subsets $e_{i}$ and $e_{j}$ are considered to be equal if the sets $\left|e_{i}\right|$ and $\left|e_{j}\right|$ are identical and the indices $i$ and $j$ are equal.

By a covering of a set we understand such a finite system of indexed subsets

$$
\Omega=\left\{e_{1}, \cdots, e_{s}\right\}
$$

that

$$
\left|e_{1}\right| \mathbf{u} \cdots \mathbf{u}\left|e_{s}\right|=R .
$$

A covering $\Omega$ is called simple if the identity $\left|e_{i}\right|=\left|e_{j}\right|$ is realized only in the case of equality $i=j$, i.e., only in the case of equality $e_{i}=e_{j}$. Since in the 
case of a simple covering the indexed sets $e_{i}$ correspond to the sets $\left|e_{i}\right|$ in oneto-one manner, we may not distinguish between the first and the latter and consider the sets $\left|e_{i}\right|$ themselves to be the elements of the simple covering.

The nerve of the covering $\Omega=\left\{e_{1}, \cdots, e_{s}\right\}$ is the complex $K$ with the vertices $e_{1}, \cdots, e_{\varepsilon}$; the vertices

$$
e_{i_{0}}, \cdots, e_{i_{r}}
$$

form a simplex of the complex $K$ when and only when

$$
\left|e_{i_{0}}\right| n \ldots n\left|e_{i_{r}}\right| \neq 0 \text {. }
$$

If a covering is denoted by the letter $\Omega$ with some indices, then we shall denote its nerve by the letter $K$ with the same indices. For instance, the nerve of the covering $\Omega^{\alpha}$ shall be denoted by $K^{\alpha}$, the nerve of the covering $\Omega^{\alpha \lambda}$ by $K^{\alpha \lambda}$, etc.

\subsection{Definition 5.21. A covering}

$$
\Omega^{\beta}=\left\{e_{j}^{\beta}\right\},
$$$$
j=1, \cdots, s_{\beta},
$$

is called a subdivision of the covering

$$
\Omega^{\alpha}=\left\{e_{i}^{\alpha}\right\},
$$$$
i=1, \cdots, s_{\alpha},
$$

if each of the $\left|e_{0}^{\beta}\right|$ is contained in at least one of the $\left|e_{i}^{\alpha}\right|$.

Definition 5.22. A covering $\Omega^{\beta}$ follows after the covering $\Omega^{\alpha}$,

$$
\Omega^{\beta}>\Omega^{\alpha},
$$

if $\Omega^{\beta}$ is a subdivision of the covering $\Omega^{\alpha}$, but $\Omega^{\alpha}$ is not a subdivision of the covering $\Omega^{\beta}$.

This definition of "follows" turns the set of all coverings $\Omega$ of a set $R$ into a partially ordered set $\mathfrak{W}$. Every part $\mathfrak{B}$ of the partially ordered set $\mathfrak{W}$ is called a system of coverings of the set $R$.

From Definition $\mathbf{5 . 2 2}$ follows

5.221. If $\Omega^{\beta}>\Omega^{\alpha}$ and $\Omega^{\gamma}$ is a subdivision of $\Omega^{\beta}$, then $\Omega^{\gamma}>\Omega^{\alpha}$; if $\Omega^{\beta}$ is a subdivision of $\Omega^{\alpha}$ and $\Omega^{\gamma}>\Omega^{\beta}$, then $\Omega^{\gamma}>\Omega^{\alpha}$.

For the nerves of the coverings we put $K^{\beta}>K^{\alpha}$, if $\Omega^{\beta}>\Omega^{\alpha}$.

Let a covering $\Omega^{\alpha}=\left\{e_{i}^{\alpha}\right\}$ and its subdivision $\Omega^{\beta}=\left\{e_{j}^{\beta}\right\}$ be given. To each $e_{j}^{\beta}$ we correlate some definite $e_{i}^{\alpha}$ under the only condition that

$$
\left|e_{j}^{\beta}\right| \subset\left|e_{i}^{\alpha}\right|
$$

Such a mapping of the covering $\Omega^{\beta}$ into the covering $\Omega^{\alpha}$ and also the corresponding simplicial mapping of the complex $K^{\beta}$ into the complex $K^{\alpha}$ shall be 
called a protection and denoted by $S_{\alpha}^{\beta}$ or, if no misunderstandings are to be feared, simply by $S$.

5.3. Suppose that a certain system $(5$ of subsets of the set $R$ called special subsets is singled out. We suppose that the system of special subsets satisfies the following condition:

5.31. If $E$ is a special subset and $E^{\prime} \supset E$, then $E^{\prime}$ is also a special subset.

The element $e_{1}^{\alpha}$ of the covering $\Omega^{\alpha}$ is called special, if $\left|e_{1}^{\alpha}\right|$ is a special subset.

Special elements of the covering $\Omega^{\alpha}$ define a certain subcomplex $C^{\alpha}$ of the complex $K^{\alpha}$ called the special subcomplex of the complex $K^{\alpha}$.

From the condition 5.31 follows

5.32. For any protection $S_{\alpha}^{\beta}$ of the covering $\Omega^{\beta}$ into the covering $\Omega^{\alpha}$ we have $S_{\alpha}^{\beta} C^{\beta} \subset C^{\alpha}$.

Let $S$ and $\tilde{S}$ be two projections of the covering $\Omega^{\beta}$ into the covering $\Omega^{\alpha}$. If

$$
t_{\beta}=\left(e_{j_{0}}, \cdots, e_{j_{r}}\right)
$$

is any simplex from $K^{\beta}$, then the vertices

$$
S e_{j_{0}}, \tilde{S} e_{j_{0}} ; \cdots ; S e_{j_{r}}, \tilde{S} e_{j_{r}}
$$

define a simplex $t_{\alpha}$ in $K^{\alpha}$ having among its faces $S t_{\beta}$ as well as $\tilde{S} t_{\beta}$; if, moreover, $t_{\beta} \varepsilon C^{\beta}$, then $t_{\alpha} \varepsilon C^{\alpha}$. Hence all projections of the covering $\Omega^{\beta}$ into the covering $\Omega^{\alpha}$ define one and the same homomorphism $\tilde{\omega}_{\alpha}^{\beta}$ of the group $B_{\Delta}^{r}\left(K^{\beta} \bmod C^{\beta}\right)$ into the group $B_{\Delta}^{r}\left(K^{\alpha} \bmod C^{\alpha}\right)$ and one and the same homomorphism $\pi_{\beta}^{\alpha}$ of the group $B_{\nabla}^{r}\left(K^{\alpha}-C^{\alpha}\right)$ into the group $B_{\nabla}^{r}\left(K^{\beta}-C^{\beta}\right)$.

If the system of coverings $\mathfrak{B}$ is unbounded, then we obtain the inverse and the direct spectra

$$
\left[B_{\Delta}^{r}\left(K^{\alpha} \bmod C^{\alpha}\right) ; \tilde{\omega}_{\alpha}^{\beta}\right], \quad\left[B_{\nabla}^{r}\left(K^{\alpha}-C^{\alpha}\right) ; \pi_{\beta}^{\alpha}\right],
$$

the limit groups of which we denote respectively by $B_{\Delta}^{r}(\mathfrak{B}, \mathfrak{E})$ and $B_{\nabla}^{r}(\mathfrak{B}, \mathfrak{E})$. From the investigations of $\operatorname{Steenrod}\left({ }^{4}\right)$ it follows that the groups $B_{\Delta}^{r}(\mathfrak{B}, \mathfrak{E})$ and $B_{\nabla}^{r}(\mathfrak{B},(\mathcal{E})$ are dual to each other.

5.4. Consider some covering, which we shall denote by

$$
\Omega^{\alpha \lambda}=\left\{e_{i k}\right\} ;
$$

by $e_{i 1}, e_{i 2}, \cdots, e_{i h_{i}}$ are denoted all elements of the covering $\Omega^{\alpha \lambda}$ for which $\left|e_{i 1}\right|=\left|e_{i 2}\right|=\cdots=\left|e_{i h_{i}}\right|$, namely $\left|e_{i k}\right|=e_{i}$. The sets $e_{i}$ form a simple covering $\Omega^{\alpha}$ denoted also by $\left|\Omega^{\alpha \lambda}\right|$.

To every element $e_{i k}$ of the covering $\Omega^{\alpha \lambda}$ there corresponds an element $e_{i}$ of the covering $\Omega^{\alpha}$ and this correspondence establishes a simplicial mapping $D_{\alpha}^{\alpha \lambda}$ of the complex $K^{\alpha \lambda}$ into the complex $K^{\alpha}$, under which the special complex 
$C^{\alpha \lambda} \subset K^{\alpha \lambda}$ is transformed into the special complex $C^{\alpha} \subset K^{\alpha}$. The simplicial mapping $D_{\alpha}^{\alpha \lambda}$ generates a homomorphism $\rho_{\alpha}^{\alpha \lambda}$ of the group $B_{\Delta}^{r}\left(K^{\alpha \lambda} \bmod C^{\alpha \lambda}\right)$ into the group $B_{\Delta}^{r}\left(K^{\alpha} \bmod C^{\alpha}\right)$ and a homomorphism $\sigma_{\alpha \lambda}^{\alpha}$ of the group $B_{\nabla}^{r}\left(K^{\alpha}-C^{\alpha}\right)$ into the group $B_{\nabla}^{r}\left(K^{\alpha \lambda}-C^{\alpha \lambda}\right)$.

5.41. The homomorphisms $\rho_{\alpha}^{\alpha \lambda}$ and $\sigma_{\alpha \lambda}^{\alpha}$ are isomorphic mappings of, correspondingly, $B_{\Delta}^{r}\left(K^{\gamma \lambda} \bmod C^{\alpha \lambda}\right)$ on $B_{\Delta}^{r}\left(K^{\alpha} \bmod C^{\alpha}\right)$ and of $B_{\nabla}^{r}\left(K^{\alpha}-C^{\alpha}\right)$ on $B_{\nabla}^{r}\left(K^{\alpha \lambda}-C^{\alpha \lambda}\right)$.

For the proof observe in the first place that the subcomplex $K^{\alpha \lambda 1}$ of the complex $K^{\alpha \lambda}$ consisting of all simplexes of $K^{\alpha \lambda}$ whose vertices have the form $e_{i 1}$ is isomorphic to the complex $K^{\alpha}$. In virtue of this isomorphism between $K^{\alpha \lambda 1}$ and $K^{\alpha}$ to the simplicial mapping $D_{\alpha}^{\alpha \lambda}$ of the complex $K^{\alpha \lambda}$ on the complex $K^{\alpha}$ there corresponds a simplicial mapping $D_{\alpha \lambda 1}^{\alpha \lambda}$ of the complex $K^{\alpha \lambda}$ on $K^{\alpha \lambda 1}$ correlating to the vertex $e_{i k}$ the vertex $e_{i 1}$ and, consequently, leaving all vertices and all simplexes of the complex $K^{\alpha \lambda 1} \subset K^{\alpha \lambda}$ fixed.

An arbitrary simplex

$$
t_{\alpha \lambda}=\left(e_{i_{0} k_{0}}, \cdots, e_{i_{r} k_{r}}\right)
$$

of the complex $K^{\alpha \lambda}$ and its image under the mapping $D_{\alpha \lambda 1}^{\alpha \lambda}$,

$$
D_{\alpha \lambda 1}^{\alpha \lambda}\left(t_{\alpha \lambda}\right)=\left(e_{i_{0} 1}, \cdots, e_{i_{r} 1}\right)
$$

are faces of a simplex

$$
T_{\alpha \lambda}=\left(e_{i_{0} 1}, \cdots, e_{i_{0} h_{0}}, \cdots, e_{i_{r},}, \cdots, e_{i_{r} h_{r}}\right)
$$

(we write $h_{0}$ instead of $h_{i_{0}}, \cdots, h_{r}$ instead of $h_{i_{r}}$ ) belonging to the complex $K^{\alpha \lambda}$ and, moreover, if $t_{\alpha \lambda} \varepsilon C^{\alpha \lambda}$, then $T_{\alpha \lambda} \varepsilon C^{\alpha \lambda}$. Hence it follows that for

we have

$$
f_{\alpha \lambda}^{r} \varepsilon Z_{\Delta}^{r}\left(K^{\alpha \lambda} \bmod C^{\alpha \lambda}\right)
$$

$$
D_{\alpha \lambda 1}^{\alpha \lambda} f_{\alpha \lambda}^{r} \sim f_{\alpha \lambda}^{r} \text { modulo } C^{\alpha \lambda}
$$

i.e., for every homologic class

we have

$$
\zeta_{\alpha \lambda}^{r} \varepsilon B_{\Delta}^{r}\left(K^{\alpha \lambda} \bmod C^{\alpha \lambda}\right)
$$

$$
\rho_{\alpha \lambda 1}^{\alpha \lambda} \zeta_{\alpha \lambda}^{r} \subset \zeta_{\alpha \lambda}^{r} .
$$

On the other hand every homologic class

$$
\zeta_{\alpha \lambda 1}^{r} \varepsilon B_{\Delta}^{r}\left(K^{\alpha \lambda 1} \bmod C^{\alpha \lambda 1}\right)
$$

is contained in a uniquely determined homologic class

$$
\zeta_{\alpha \lambda}^{r} \varepsilon B_{\Delta}^{r}\left(K^{\alpha \lambda} \bmod C^{\alpha \lambda}\right) \text {. }
$$


It remains to show that the homologic class $\zeta_{\alpha \lambda}^{r}$ contains only one homologic class $\zeta_{\alpha \lambda 1}^{r}$, i.e., that from

$$
f_{\alpha \lambda 1}^{r} \varepsilon Z_{\Delta}^{r}\left(K^{\alpha \lambda 1} \bmod C^{\alpha \lambda 1}\right), \quad f_{\alpha \lambda 1}^{r} \varepsilon H_{\Delta}^{r}\left(K^{\alpha \lambda} \bmod C^{\alpha \lambda}\right)
$$

follows

$$
f_{\alpha \lambda 1}^{r} \varepsilon H_{\Delta}^{r}\left(K^{\alpha \lambda 1} \bmod C^{\alpha \lambda 1}\right) .
$$

Since under our assumptions

$$
f_{\alpha \lambda 1}^{r}=\Delta f_{\alpha \lambda}^{r+1}
$$

we have

$$
f_{\alpha \lambda 1}^{r}=D_{\alpha \lambda 1}^{\alpha \lambda} f_{\alpha \lambda 1}^{r}=D_{\alpha \lambda 1}^{\alpha \lambda} \Delta f_{\alpha \lambda}^{r+1}=\Delta D_{\alpha \lambda 1}^{\alpha \lambda} f_{\alpha \lambda}^{r+1},
$$

so that the inclusion (5.41) is proved.

Thus $\rho_{\alpha}^{\alpha \lambda}$ is an isomorphic mapping of the group $B_{\Delta}^{r}\left(K^{\alpha \lambda} \bmod C^{\alpha \lambda}\right)$ on $B_{\Delta}^{r}\left(K^{\alpha} \bmod C^{\alpha}\right)$, consequently $\sigma_{\alpha \lambda}^{\alpha}$ is an isomorphic mapping of the group $B_{\nabla}^{r}\left(K^{\alpha}-C^{\alpha}\right)$ on $B_{\nabla}^{r}\left(K^{\alpha \lambda}-C^{\alpha \lambda}\right)$.

5.5. Let there be given coverings $\Omega^{\alpha \lambda}, \Omega^{\alpha}, \Omega^{\beta \mu}, \Omega^{\beta}$, connected by the relations

$$
\Omega^{\alpha}=\left|\Omega^{\alpha \lambda}\right|, \quad \Omega^{\beta}=\left|\Omega^{\beta \mu}\right| ; \quad \Omega^{\beta \mu}>\Omega^{\alpha \lambda} .
$$

Then also $\Omega^{\beta}>\Omega^{\alpha}$ and, moreover, if $S_{\alpha \lambda}^{\beta \mu}$ is a projection of $\Omega^{\beta \mu}$ into $\Omega^{\alpha \lambda}$, then we have a completely determined projection $S_{\alpha}^{\beta}$ defined by the formula

$$
S_{\alpha}^{\beta} e_{j}^{\beta}=S_{\alpha}^{\beta} D_{\beta}^{\beta \mu} e_{j 1}^{\beta \mu}=D_{\alpha}^{\alpha \lambda} S_{\alpha \lambda}^{\beta \mu} e_{j 1}^{\beta \mu} .
$$

Denoting by $\rho_{\alpha}^{\alpha \lambda}, \rho_{\beta}^{\beta \mu}, \sigma_{\alpha \lambda}^{\alpha}, \sigma_{\beta \mu}^{\beta}$ the isomorphisms (defined in 5.4) generated by the mappings $D_{\alpha}^{\alpha \lambda}$ and $D_{\beta}^{\beta \mu}$, we see that $\tilde{\omega}_{\alpha}^{\beta} \rho_{\beta}^{\beta \mu}$ and $\rho_{\alpha}^{\alpha \lambda} \tilde{\omega}_{\alpha \lambda}^{\beta \mu}$ is one and the same homomorphism of the group $B_{\Delta}^{r}\left(K^{\beta \mu} \bmod C^{\beta \mu}\right)$ into the group $B_{\Delta}^{r}\left(K^{\alpha} \bmod C^{\alpha}\right)$ and, consequently, $\sigma_{\beta \mu}^{\beta} \pi_{\beta}^{\alpha}$ and $\pi_{\beta \mu}^{\alpha \lambda} \sigma_{\beta \mu}^{\beta}$ express one and the same homomorphism of the group $B_{\nabla}^{\tau}\left(K^{\alpha}-C^{\alpha}\right)$ into the group $B_{\nabla}^{r}\left(K^{\beta \mu}-C^{\beta \mu}\right)$. Hence

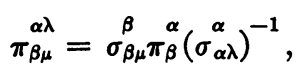

or, denoting by $\phi_{\alpha}^{\alpha \lambda}, \phi_{\mu}^{\beta \mu}$ the isomorphic mappings respectively inverse to the isomorphisms $\sigma_{\alpha \lambda}^{\alpha}$ and $\sigma_{\beta \mu}^{\beta}$,

$$
\pi_{\beta \mu}^{\alpha \lambda}=\left(\phi_{\beta}^{\beta \mu}\right)^{-1} \underset{\pi_{\beta}}{\alpha} \phi_{\alpha}^{\alpha \lambda} .
$$

5.6. Suppose now that we have an unbounded system $\mathfrak{B}$ of coverings $\Omega^{\alpha \lambda}$ of the set $R$. Let the system

$$
|\mathfrak{B}|=\left\{\Omega^{\alpha}\right\},
$$

where $\Omega^{\alpha}=\left|\Omega^{\alpha \lambda}\right|$ be also unbounded. Under these conditions we have 
5.61. The groups

$$
\begin{aligned}
B_{\Delta}^{r}(\mathfrak{B}, \mathfrak{S}) & =\lim \left[B_{\nabla}^{r}\left(K^{\alpha \lambda}-C^{\alpha \lambda}\right) ; \pi_{\beta \mu}^{\alpha \lambda}\right], \\
B_{\nabla}^{r}(|\mathfrak{B}|, \mathfrak{S}) & =\lim \left[B_{\nabla}^{r}\left(K^{\alpha}-C^{\alpha}\right) ; \pi_{\beta}^{\alpha}\right]
\end{aligned}
$$

are isomorphic.

In fact, in virtue of (5.52) the conditions of 3.61 are satisfied.

Let us establish an important particular case of 5.61.

Let $A$ be any subset of the set $R$. Suppose that, the condition 5.31 being satisfied, some subsets of the set $A$ are singled out as special. Denote the system of special subsets of the set $A$ by $\mathfrak{S}_{0}$.

Let there be given an unbounded system $\mathfrak{B}$ of coverings $\Omega^{\alpha}$ of the set $R$. We construct for each $\Omega^{\alpha}=\left\{e_{i}^{\alpha}\right\}$ a covering $A \Omega^{\alpha}$ of the set $A$ in the following manner. The elements of the covering $A \Omega^{\alpha}$ are the indexed sets

$$
\left(A \cap\left|e_{i}^{\alpha}\right|\right)_{i}
$$

which we shall simply denoted by $A e_{i}^{\alpha}$.

If $\Omega^{\beta}>\Omega^{\alpha}$, put $A \Omega^{\beta}>A \Omega^{\alpha}$. The obtained system of coverings $A \Omega^{\alpha}$ we shall denote by $A \mathfrak{B}$.

The system $|A \mathfrak{B}|$ consists of all simple coverings $\left|A \Omega^{\alpha}\right|$, where $\left|A \Omega^{\alpha}\right|$ may be defined as the simple covering consisting of all non-void sets representable in the form $A \cap\left|e_{i}^{\alpha}\right|$, where $e_{i}^{\alpha} \varepsilon \Omega^{\alpha}$.

From what has been proved above follows

5.611. If for an unbounded system of coverings $V$ of the set $R$ and for $a$ subset $A \subset R$ the system $|A \mathfrak{B}|$ is also unbounded, then the groups $B_{\nabla}^{r}\left(A V, \mathfrak{S}_{0}\right)$ and $B_{\nabla}^{r}\left(|A \mathfrak{B}|, \mathfrak{S}_{0}\right)$ are isomorphic.

\section{Coverings of topological SPACES}

6.1. A covering of a topological space is called open if it is composed of open sets, and closed if it is composed of closed sets.

6.11. Every two open (closed) coverings $\Omega^{\alpha}$ and $\Omega^{\beta}$ of a topological space $R$ have a common simple subdivision $\Omega^{\gamma}$.

It is sufficient to take for the elements of the covering $\Omega^{\gamma}$ the sets $\left|e_{i}^{\alpha}\right| \cap\left|e_{j}^{\beta}\right|$, where $e_{i}^{\alpha} \varepsilon \Omega^{\alpha}, e_{j}^{\beta} \varepsilon \Omega^{\beta}$.

6.121. Let

$$
\Omega^{\alpha}=\left\{e_{i}^{\alpha}\right\}, \quad i=1,2, \cdots, s_{\alpha},
$$

be an open covering of a $T_{1}$-space $R$. If at least one of the sets $e_{i}^{\alpha}$ contains more than one point, then there exists an open covering $\Omega^{\beta}=\left\{e_{j}^{\beta}\right\}$ following $\Omega^{\alpha}$,

$$
\Omega^{\beta}>\Omega^{\alpha} \text {. }
$$


In fact, let, for instance, $e_{1}^{\alpha}$ contain at least two points $a$ and $a^{\prime}$. Put

$$
\begin{aligned}
e_{i}^{\beta} & =e_{i}^{\alpha}-a \text { for } i \leqq s_{\alpha}, \\
e_{i}^{\beta} & =e_{1}^{\alpha}-a \text { for } i=s_{\alpha}+1 .
\end{aligned}
$$

The so-defined covering $\Omega^{\beta}=\left\{e_{i}^{\beta}\right\}, i=1,2, \cdots, s_{\alpha}+1$, is evidently a subdivision of the covering $\Omega^{\alpha}$. But $\Omega^{\alpha}$ is not a subdivision of $\Omega^{\beta}$, since $e_{1}^{\alpha}$ is not contained in any of the sets $e_{i}^{\beta}$.

6.122. Let $R$ be a $T_{1}$-space consisting of an infinite number of points. Then every closed covering

$$
\Omega^{\alpha}=\left\{e_{i}^{\alpha}\right\}, \quad i=1,2, \cdots, s_{\alpha},
$$

of $R$ possesses a subdivision $\Omega^{\beta}$, containing an element with an infinite number of points not belonging to any other element of the covering $\Omega^{\beta}$.

In fact, let us delete from (6.1) one after another all elements, all points of which with the possible exception of a finite number of them are contained in the sum of the following elements of the covering $\Omega^{\alpha}$. At every such deletion we lose not more than a finite number of points of the space $R$. Hence, if $R$ consists of an infinite number of points, we shall at last reach such a first element $e_{i}$ that the set $O_{i}=e_{i}-\left(e_{i+1} \cup \cdots \cup e_{s_{\alpha}}\right)$ contains infinitely many points. If at the preceding deletion we lost the finite set of points $p_{1}, \cdots, p_{h}$, then

$$
\left\{p_{1}, \cdots, p_{h}, e_{i}^{\alpha}, e_{i+1}^{\alpha}, \cdots, e_{s_{\alpha}}^{\alpha}\right\}
$$

is the required subdivision of the covering $\Omega^{\alpha}$.

6.123. Let $R$ be a $T_{2}$-space consisting of an infinite number of points. For every closed covering

$$
\Omega^{\alpha}=\left\{e_{i}^{\alpha}\right\}, \quad i=1,2, \cdots, s_{\alpha}
$$

of the space $R$ there is a covering

$$
\Omega^{\beta}=\left\{e_{j}^{\beta}\right\}
$$

following after $\Omega^{\alpha}$.

In fact, we may suppose that $\Omega^{\alpha}$ satisfies the conditions of 6.122 and that, for instance,

$$
O_{1}=e_{1}^{\alpha}-\bigcup_{2 \leqq i \leqq s_{\alpha}} e_{i}^{\alpha}
$$

contains infinitely many points. Take two points $a$ and $a^{\prime}$ of the set $O_{1}$ and choose such a neighbourhood $O_{a}$ of the point $a$ that

$$
\bar{O}_{a} \subset O_{1}-a^{\prime} \text {. }
$$


Since $O_{1}$ is an open set, such a neighbourhood may be found. Put now

$$
\begin{aligned}
e_{i}^{\beta} & =e_{i}^{\alpha}-O_{a}, & \text { if } & i \leqq s_{\alpha}, \\
e_{i}^{\beta} & =\bar{O}_{a}, & & \text { if } i=s_{\alpha}+1 .
\end{aligned}
$$

The covering $\Omega^{\beta}=\left\{e_{i}^{\beta}\right\}, i=1,2, \cdots, s_{\alpha}+1$, is the required covering.

From what has been proved above follows

6.12. The system of all open coverings of every $T_{1}$-space consisting of an infinite number of points and the system of all closed coverings of every $T_{2}$-space consisting of an infinite number of points are unbounded systems of coverings.

6.2. From now on and until the end of the present paper we shall suppose, if the contrary is not explicitly stated, that $R$ is a normal space consisting of an infinite number of points. By a covering of the space $R$ we shall always mean an open covering. The system of all open coverings of the space $R$ we shall denote by $\mathfrak{D}$.

By $A$ we always denote a closed set lying in the space $R$. $A$ itself is a normal space, which, in general, cannot be asserted with respect to $R-A$.

In every covering $\Omega=\left\{e_{i}\right\}, i=1,2, \cdots, s$, of the space $R$ we distinguish:

$1^{\circ}$. Elements of the first kind, i.e., elements meeting $A$; we denote them by $e_{1}, \cdots, e_{p}$.

$2^{\circ}$. Elements of the second kind, $e_{p+1}, \cdots, e_{s}$, not meeting $A$.

The elements of the second kind are subdivided into boundary elements:

$$
e_{p+1}, \cdots, e_{q},
$$

satisfying the condition $A \cap \bar{e}_{i} \neq 0, i=p+1, \cdots, q$, and inner elements:

$$
e_{q+1}, \cdots, e_{s},
$$

for which $\bar{e}_{i} \subset R-A, i=q+1, \cdots, s$.

Definition 6.21. A covering

$$
\Omega=\left\{e_{i}\right\}, \quad i=1,2, \cdots, s,
$$

of the space $R$ is called regular with respect to $A$, if it satisfies the following conditions:

$1^{\circ}$. The covering $\Omega$ contains no boundary elements of second kind.

$2^{\circ}$. If for some elements of the first kind $e_{i_{0}}, \cdots, e_{i_{r}}$ we have

$$
A \cap e_{i_{0}} \cap \cdots \cap e_{i_{r}}=0,
$$

then

$$
\bar{e}_{i_{0}} n \cdots n \bar{e}_{i_{r}}=0 .
$$

Observe that from these conditions follows 
$3^{\circ}$. If for any elements $e_{i_{0}}, \cdots, e_{i}$ of the covering $\Omega$ we have

$$
A \cap e_{i_{0}} \cap \cdots \cap e_{i_{r}}=0,
$$

then

$$
A \cap \bar{e}_{i_{0}} \cap \cdots \cap \bar{e}_{i_{r}}=0
$$

6.22. Every covering

$$
\Omega^{\alpha}=\left\{e_{i}^{\alpha}\right\}, \quad i=1,2, \cdots, s_{\alpha},
$$

of the space $R$ has a subdivision $\Omega^{\beta}$ regular with respect to $A$.

Proof. Consider the open covering

$$
A \Omega^{\alpha}=\left\{A e_{1}^{\alpha}, \cdots, A e_{p}^{\alpha}\right]
$$

of the set $A$. Since $A$ is a normal space, there exists a closed covering

$$
a_{1}, \cdots, a_{p}
$$

of the set $A$ similar to it and inscribed into (6.21). About the system of closed sets (6.22) we circumscribe a system of sets

$$
O a_{1}, \cdots, O a_{p}
$$

open in $R$ and similar to it such that for $j=1,2, \cdots, p$

$$
a_{j} \subset O a_{j} \subset \stackrel{\alpha}{e_{j}} .
$$

For each $a_{j}$ take a neighbourhood $O^{\prime} a_{j}$ such that

$$
\overline{O^{\prime} a_{j}} \subset O a_{j}
$$

and put

$$
e_{j}^{\beta}=O^{\prime} a_{j}, \quad j=1,2, \cdots, p .
$$

Take further a neighbourhood $O^{\prime \prime} A$ of the set $A$ such that

$$
\overline{O^{\prime \prime} A} \subset \underset{1 \leqq j \leqq p}{\bigcup} e_{j}^{\beta}
$$

and denote the non-void sets among the $e_{i}^{\alpha}-\overline{O^{\prime \prime} A}$ by $e_{j}^{\beta}, j=p+1, \cdots, s_{\beta}$.

These last exhaust all elements of the second kind of the covering

$$
\Omega^{\beta}=\left\{e_{j}^{\beta}\right\}, \quad j=1,2, \cdots, s_{\beta},
$$

and all these elements are evidently inner elements.

Thus the covering $\Omega^{\beta}$ satisfies the first condition of regularity with respect to $A$.

Let us prove that the second condition of regularity is also satisfied. Sup- 
pose that for certain elements of the first kind, which we shall for simplicity's sake denote by $e_{1}^{\beta}, \cdots, e_{r}^{\beta}$, we have

$$
A \cap e_{1}^{\beta} \cap \cdots \cap e_{r}^{\beta}=0
$$

and, consequently, also

$$
A e_{1}^{\beta} \cap \cdots \cap A e_{r}^{\beta}=0 .
$$

Since $a_{i} \subset A e_{i}^{\beta}$, we have also $a_{1} \cap \ldots \cap a_{r}=0$ and, consequently also

$$
O a_{1} \cap \cdots \cap O a_{r}=0 .
$$

And moreover

$$
\overline{O^{\prime} a_{1}} \mathrm{n} \cdots \overline{\mathrm{n}} \overline{O^{\prime} a_{r}}=0
$$

i.e.,

$$
\bar{e}_{1}^{\beta} \cap \cdots \cap \bar{e}_{r}^{\beta}=0 .
$$

Since $\Omega^{\beta}$ is by very construction a subdivision of $\Omega^{\alpha},(6.22)$ is proved.

6.3. Consider in any covering $\Omega^{\alpha}$ of the space $R$ the set of elements of the first kind and of boundary elements of the second kind. These elements considered as vertices of the nerve $K^{\alpha}$ of the covering $\Omega^{\alpha}$ define in $K^{\alpha}$ a subcomplex $K_{1}^{\alpha}$. By $K_{0}^{\alpha}$ we as always understand the nerve of the covering $A \Omega^{\alpha}$ considered as a subcomplex of the complex $K^{\alpha}$. Evidently we always have $K_{0}^{\alpha} \subset K_{1}^{\alpha}$.

6.31. If a covering $\Omega^{\alpha}$ is regular with respect to $A$, then $K_{1}^{\alpha}=K_{0}^{\alpha}$.

In fact, from the first condition of regularity it follows that the complexes $K_{1}^{\alpha}$ and $K_{0}^{\alpha}$ have the same vertices, while from the second condition it follows that every simplex of $K_{1}^{\alpha}$ is at the same time a simplex of $K_{0}^{\alpha}$.

At every projection of the covering $\Omega^{\beta}$ into the covering $\Omega^{\alpha}$ the complex $K_{1}^{\beta}$ is obviously transformed into $K_{1}^{\alpha}$; hence we may speak of the spectrum

$$
\left[B_{\nabla}^{r}\left(K^{\alpha}-K_{1}^{\alpha}-C^{\alpha}\right) ; \pi_{\beta}^{\alpha}\right]
$$

where, as always, $C^{\alpha}$ denotes the special subcomplex of the complex $K^{\alpha}$.

The elements of the spectrum (6.31) corresponding to coverings $\Omega^{\alpha}$ regular with respect to $A$ form in virtue of 6.22 a cofinal part of this spectrum. Hence, having in view 6.31 , we obtain

6.32. The groups

$$
\begin{aligned}
& \lim \left[B_{\nabla}^{r}\left(K^{\alpha}-K_{0}^{\alpha}-C^{\alpha}\right) ; \pi_{\beta}^{\alpha}\right], \\
& \lim \left[B_{\nabla}^{r}\left(K^{\alpha}-K_{1}^{\alpha}-C^{\alpha}\right) ; \pi_{\beta}^{\alpha}\right]
\end{aligned}
$$

are isomorphic. 


\section{BETTI GROUPS OF TOPOLOGICAL SPACES (FIRST DEFINITION)}

7.1. Let $R$ be a normal space consisting of an infinite number of points. Let $\mathfrak{D}=\left\{\Omega^{\alpha}\right\}$ be the system of all open coverings of the space $R$. By $K^{\alpha}$ we always denote the nerve of the covering $\Omega^{\alpha}$.

Definition 7.11. The group $\left(^{9}\right)$

$$
\lim \left[B_{\nabla}^{\gamma}\left(K^{\alpha}\right) ; \pi_{\beta}^{\alpha}\right],
$$

where $\pi_{\beta}^{\alpha}$ is a projection of the group $B_{\nabla}^{r}\left(K^{\alpha}\right)$ into the group $B_{\nabla}^{r}\left(K^{\beta}\right)$, generated by any projection of the covering $\Omega^{\beta}$ into the covering $\Omega^{\alpha}$ will be denoted by $\mathfrak{B}_{\nabla}^{r}(R)$.

7.2. Let $G$ be a topological space consisting of an infinite number of points and homeomorphic to an open set of a certain normal space (in particular, for $G$ may be taken any normal space). By a special subset of the space $G$ we shall mean any set $E \subset G$ whose closure in $G$ is not bicompact. The system $\mathbb{5}$ of special subsets of the space $G$ obviously satisfies the condition 5.31: any set $E \subset G$ containing a special subset is itself special. By $\mathfrak{D}=\left\{\Omega^{\alpha}\right\}$ we denote the system of all open coverings of the space $G$. Special elements of the covering $\Omega^{\alpha}$, i.e., elements $e_{i}^{\alpha}$, for which $\left|e_{i}^{\alpha}\right|$ is a special subset, determine the special subcomplex $C^{\alpha}$ of the complex $K^{\alpha}$ : the complex $C^{\alpha} \subset K^{\alpha}$ consists of simplexes of the complex $K^{\alpha}$, all vertices of which are special elements of $\Omega^{\alpha}$.

DeFinition 7.2. The $\operatorname{group}\left({ }^{9}\right)$

$$
\lim \left[B_{\nabla}^{r}\left(K^{\alpha}-C^{\alpha}\right) ; \pi_{\beta}^{\alpha}\right],
$$

where $\pi_{\beta}^{\alpha}$ is a projection of the group $B_{\nabla}^{r}\left(K^{\alpha}-C^{\alpha}\right)$ into $B_{\nabla}^{r}\left(K^{\beta}-C^{\beta}\right)$, generated by any projection of the covering $\Omega^{\beta}$ into the covering $\Omega^{\alpha}$ is called the $r$-dimensional (inner) Betti $\nabla$-group of the space $G$ and is denoted by $B_{\nabla}^{r}(G)$.

7.3. The field of coefficients forming the foundation of the above definitions is, as always in the $\nabla$-theory, supposed to be a discrete commutative group $X$. If $\Xi$ is the bicompact group dual to $X$ and

$$
B_{\Delta}^{r}\left(K^{\alpha}\right)=B_{\Delta}^{r}\left(K^{\alpha}, \Xi\right), \quad B_{\Delta}^{r}\left(K^{\alpha} \bmod C^{\alpha}\right)=B_{\Delta}^{r}\left(K^{\alpha} \bmod C^{\alpha}, \Xi\right)
$$

then the limit groups

$$
\begin{aligned}
& B_{\Delta}^{r}(R)=\lim _{\leftarrow}\left[B_{\nabla}^{r}\left(K^{\alpha}\right) ; \tilde{\omega}_{\alpha}^{\beta}\right], \\
& B_{\Delta}^{r}(R)=\lim _{\leftarrow}\left[B_{\Delta}^{r}\left(K^{\alpha} \bmod C^{\alpha}\right) ; \tilde{\omega}_{\alpha}^{\beta}\right]
\end{aligned}
$$

(where $\tilde{\omega}_{\alpha}^{\beta}$ is the homomorphism of the group $B_{\Delta}^{r}\left(K^{\beta}\right)$ into $B_{\Delta}^{r}\left(K^{\alpha}\right)$, respectively of the group $B_{\Delta}^{r}\left(K^{\beta} \bmod C^{\beta}\right)$ into $B_{\Delta}^{r}\left(K^{\alpha} \bmod C^{\alpha}\right)$, generated by the projection

( ${ }^{9}$ See 4.8 . 
of $K^{\beta}$ into $K^{\alpha}$ ) are dual to, respectively, the groups $B_{\nabla}^{r}(R)$ and $B_{\nabla}^{r}(R)$; the group $B_{\Delta}^{r}(R)$ is called the $r$-dimensional (inner) Betti $\Delta$-group of the space $R$.

Remark. The simple coverings obviously form a cofinal part of the system of all coverings. In the definitions just given we may therefore always assume that all coverings are simple.

7.4. THEOREM 7.41. Let each of the groups $\mathfrak{B}_{\nabla}^{r}(R)$ and $\mathfrak{B}_{\nabla}^{r+1}(R)$ consist of the zero element only. Let $A$ be a closed set of the space $R$. Then $\mathfrak{B}_{\nabla}^{r}(A)$ is isomorphic to the groups

$$
\lim \left[B_{\nabla}^{r+1}\left(K^{\alpha}-K_{0}^{\alpha}\right] ; \pi_{\beta}^{\alpha}\right), \quad \lim \left[B_{\nabla}^{r+1}\left(K^{\alpha}-K_{1}^{\alpha}\right) ; \pi_{\beta}^{\alpha}\right],
$$

where the complexes $K_{0}^{\alpha}$ and $K_{1}^{\alpha}$ are defined as in 6.3.

Proof. In the first place, for $\subseteq=0$ in virtue of 6.32 the groups

$$
\lim \left[B_{\nabla}^{r+1}\left(K^{\alpha}-K_{0}^{\alpha}\right) ; \pi_{\beta}^{\alpha}\right], \quad \lim \left[B_{\nabla}^{r+1}\left(K^{\alpha}-K_{1}^{\alpha}\right) ; \pi_{\beta}^{\alpha}\right]
$$

are isomorphic, and hence it is sufficient to show that $\mathfrak{B}_{\nabla}^{r}(A)$ is isomorphic to the group

$$
\lim \left[B_{\nabla}^{r+1}\left(K^{\alpha}-K_{0}^{\alpha}\right) ; \pi_{\beta}^{\alpha}\right] .
$$

But in virtue of Theorem 4.1 this last group is isomorphic to the group $\mathfrak{B}_{\nabla}^{r}(A D)$. Thus, everything is reduced to the proof of the following

LEMMA 7.411. The group $\mathfrak{B}_{\nabla}^{r}(A)$ is isomorphic to the group $\mathfrak{B}_{\nabla}^{r}(A \mathfrak{D})$.

We begin the proof of Lemma 7.411 with the consideration of the case when $A$ consists of a finite number of points.

Consider coverings $\Omega^{\alpha}$ of the space $R$ satisfying the following conditions:

$1^{\circ}$. The covering $\Omega^{\alpha}$ is a simple covering.

$2^{\circ}$. Every element of $\Omega^{\alpha}$ contains not more than one point of $A$.

$3^{\circ}$. Two different elements of $\Omega^{\alpha}$ containing points of $A$ do not meet (in particular, no two elements of $\Omega^{\alpha}$ contain one and the same point of $A$ ).

It is easily seen that every covering of $R$ has a subdivision satisfying the conditions $1^{\circ}-3^{\circ}$, so that the system $\mathfrak{B}$ of coverings satisfying these conditions forms a cofinal part of the system $\varrho$ of all coverings $\Omega^{\alpha}$. But if $\Omega^{\alpha}$ satisfies the conditions $1^{\circ}-3^{\circ}$, then $A \Omega^{\alpha}$ has for its elements the points of $A$ themselves, and the nerve $K_{0}^{\alpha}$ of the covering $A \Omega^{\alpha}$ is a zero-dimensional complex which may be identified with the same finite set $A$. Hence $B_{\nabla}^{r}\left(K_{0}^{\alpha}\right)=B_{\nabla}^{r}(A)$; in the spectrum

$$
\left[B_{\nabla}^{r}\left(K_{0}^{\alpha}\right) ; \pi_{\beta}^{\alpha}\right]
$$

the projections $\pi_{\beta}^{\alpha}$ are identical mappings of $B_{\nabla}^{r}(A)$ onto itself and, consequently, the group $B_{\nabla}^{r}(A \mathfrak{D})$, being isomorphic to $B_{\nabla}^{r}(A \mathfrak{B})$, is also isomorphic to $B_{\nabla}^{r}(A)$. 
Let now $A$ be infinite. For $\mathfrak{S}_{0}=0$ in virtue of 5.611 the groups $\mathfrak{B}_{\nabla}^{r}(A \mathfrak{D})$ and $\mathfrak{B}_{\nabla}^{r}(|A \mathfrak{D}|)$ are isomorphic. Hence it is sufficient to show

7.42. The group $\mathfrak{B}_{\nabla}^{r}(A)$ is isomorphic to the group $B_{\nabla}^{r}(|A \mathfrak{D}|)$.

This proposition follows in its turn from

7.43. The system of all simple coverings of the space $A$ coincides with the system $|A \mathfrak{O}|$.

To prove 7.43 it is sufficient to show that every simple open covering

$$
\Omega_{A}=\left\{a_{i}\right\}, \quad i=1,2, \cdots, s,
$$

of the set $A$ is an element of the system $|A \mathfrak{D}|$. To this end choose for every $a_{i} \varepsilon \Omega_{A}$ a set $e_{i}$ open in $R$ such that $a_{i}=A \cap e_{i}$. The sets $e_{i}$ in their sum form a certain neighbourhood $O A$ of the set $A$. Choose a neighbourhood $O^{\prime} A$ such that $\overline{O^{\prime} A} \subset O A$ and put

$$
e_{s+1}=R-\overline{O^{\prime} A} \text {. }
$$

Denoting by $\Omega \varepsilon \mathfrak{D}$ the covering $\left\{e_{i}\right\}, i=1,2, \cdots, s$, of the space $R$, we evidently have

$$
\Omega_{A}=|A \Omega| \text {, }
$$

which proves 7.43 and, consequently, 7.42 and 7.41 .

7.5. Let now $R$ be a locally bicompact normal space, $A$ an infinite closed subset of the space $R$. Special subsets of the set $R$ (in particular, of the set $A$ ) shall be as above sets, the closures of which are not bicompact. Let

$$
\Omega=\left\{e_{1}, \cdots, e_{s}\right\}
$$

be a covering of $R$. An element $e_{i} \varepsilon \Omega$ we shall for a moment call unregular if $\bar{e}_{i}$ is not bicompact and $A \cap \bar{e}_{i}$ is bicompact and non-void.

7.51. Every covering (7.51) has a subdivision not containing any unregular element.

For the proof it is sufficient to construct for every covering $\Omega$ containing unregular elements such a subdivision $\Omega_{1}$ that the number of unregular elements in $\Omega_{1}$ should be by unity less than the number of unregular elements in $\Omega$.

Let $e_{1}$ be an unregular element of the covering $\Omega$. Since $\overline{\mathrm{An}_{1}}$ is bicompact, we may, using the local bicompactness of $R$, construct such a neighbourhood $U_{0}$ of $\overline{A \cap e_{1}}$ that $\bar{U}_{0}$ is bicompact. The bicompact set $\bar{U}_{0}$ may be again enclosed into a neighbourhood $U_{1}$ with a bicompact closure. Put

$$
e_{11}=e_{1} \cap U_{1}, \quad e_{12}=e_{1}-\bar{U}_{0}, \quad \Omega_{1}=\left\{e_{11}, e_{12}, e_{2}, \cdots, e_{s}\right\} .
$$

Since $e_{11}$ and $e_{12}$ are regular, $\Omega_{1}$ is the required subdivision. 
7.52. Let there be given a covering $\Omega$ of the space $R$. As usual denote by $K$ the nerve of the covering $\Omega$, by $K_{0}$ the nerve of the covering $A \Omega$, and by $C$ and $C_{0}$ special subcomplexes of the complexes $K$ and $K_{0}$. If the covering $\Omega$ does not contain unregular elements, then $C_{0}=K_{0} \cap C$.

In fact, from 7.51 it follows that the complexes $C_{0}$ and $K_{0} \cap C$ have the same vertices, which correspond in one-to-one manner to special elements of the covering $\Omega$ meeting $A$. Since in $C_{0}$ as well as in $K_{0} \cap C$ the vertices $e_{i_{0}}, \cdots, e_{i_{r}}$ determine a simplex, if

$$
A \cap e_{i_{0}} \cap \cdots \cap e_{i_{r}} \neq 0,
$$

7.52 is proved.

Definition 7.53. A locally bicompact space $R$ is said to be simply connected with respect to the dimensionality $r$, if the group $B_{\nabla}^{r}(R)$ consists of the zero element only.

THEOREM 7.54. Let $R$ be a locally bicompact normal space simply connected with respect to the dimensionalities $r$ and $r+1$. Let $A$ be a closed set of the space $R$. Then the group $B_{\nabla}^{r}(A)$ is isomorphic to the groups

$$
\lim \left[B_{\nabla}^{r+1}\left(K^{\alpha}-K_{0}^{\alpha}-C^{\alpha}\right) ; \pi_{\beta}^{\alpha}\right], \quad \lim \left[B_{\nabla}^{r+1}\left(K^{\alpha}-K_{1}^{\alpha}-C^{\alpha}\right) ; \pi_{\beta}^{\alpha}\right] .
$$

Proof. Let first $A$ consist of an infinite number of points. In virtue of 7.43 the group $B_{\nabla}^{r}(A)$ is isomorphic to the group $B_{\nabla}^{r}\left(|A \mathfrak{D}|, \mathfrak{G}_{0}\right)$ (where $\mathfrak{C}_{0}$ denotes the system of special subsets of the set $A$ ) and, consequently, on ground of 5.611 , to the group

$$
B_{\nabla}^{r}\left(A \mathfrak{D}, \mathfrak{S}_{0}\right)=\lim \left[B_{\nabla}^{r}\left(K_{0}^{\alpha}-C_{0}^{\alpha}\right) ; \pi_{\beta}^{\alpha}\right] .
$$

On ground of 7.51 and 7.52 for the cofinal part of the spectrum

$$
\left[B_{\nabla}^{r}\left(K_{0}^{\alpha}-C_{0}^{\alpha}\right) ; \pi_{\beta}^{\alpha}\right]
$$

(corresponding to the coverings $\Omega^{\alpha}$ not containing unregular elements)

$$
K_{0}^{\alpha}-C_{0}^{\alpha}=K_{0}^{\alpha}-C^{\alpha}
$$

so that $\lim \left[B_{\nabla}^{r}\left(K_{0}^{\alpha}-C_{0}^{\alpha}\right) ; \pi_{\beta}^{\alpha}\right]$ and consequently also $B_{\nabla}^{r}(A)$ are isomorphic to $\lim \left[B_{\nabla}^{r}\left(K_{0}^{\alpha}-C^{\alpha}\right) ; \pi_{\beta}^{\alpha}\right]$. But this last group is isomorphic in virtue of Theorem 4.1 to the group

$$
\lim \left[B_{\nabla}^{\boldsymbol{r}}\left(K^{\alpha}-K_{0}^{\alpha}-C^{\alpha}\right) ; \pi_{\beta}^{\alpha}\right]
$$

and, consequently, on ground of 6.32 , also to the group

q.e.d.

$$
\lim \left[B_{\nabla}^{\gamma}\left(K^{\alpha}-K_{1}^{\alpha}-C^{\alpha}\right) ; \pi_{\beta}^{\alpha}\right],
$$


Let now $A$ be finite. Then $C_{0}=0$ and, by Lemma 7.411,

$$
B_{\nabla}^{r}(A)=B_{\nabla}^{r}(A)=B_{\nabla}^{r}(A O) \text {. }
$$

For coverings $\Omega^{\alpha}$ not containing unregular elements we have

$$
K_{0}^{\alpha}=K_{0}^{\alpha}-C^{\alpha},
$$

so that the group $B_{\nabla}^{r}(A)$ is isomorphic to the group

$$
\lim \left[B_{\nabla}^{\gamma}\left(K_{0}^{\alpha}-C^{\alpha}\right) ; \pi_{\beta}^{\alpha}\right] .
$$

The rest of the proof is the same as in the case of an infinite $A$.

7.6. TheOREM 7.6. Let $R$ be a connected bicompact space simply-connected with respect to the dimensionality 1 . For any closed set $A \subset R$ the group $B_{\nabla}^{00}(A)$ is isomorphic to the groups

$$
\lim \left[B_{\nabla}^{1}\left(K^{\alpha}-K_{0}^{\alpha}\right) ; \pi_{\beta}^{\alpha}\right], \quad \lim \left[B_{\nabla}^{1}\left(K^{\alpha}-K_{1}^{\alpha}\right) ; \pi_{\beta}^{\alpha}\right] .
$$

For the proof observe in the first place that from the connectivity of $R$ follows the connectivity of all complexes $K^{\alpha}$. Noting this, we construct the proof of Theorem 7.6 precisely on the same lines as the proof of Theorem 7.54 with the only deviation that instead of taking reference to Theorem 4.1 we now apply Theorem 4.6.

\section{The duality law of KolmogorofF}

8.1. By the duality law of Kolmogoroff we mean the set of the two following theorems, the proof of which is the object of the present section.

THEOREM 8.11. Let $r$ be a natural number, $R$ a locally bicompact normal space simply connected with respect to the dimensionalities $r$ and $r+1$. For any closed set $A \subset R$ the groups $B_{\nabla}^{r}(A)$ and $B_{\nabla}^{r+1}(R-A)$ are isomorphic.

THEOREM 8.12. Let $R$ be a connected bicompact normal space simply connected with respect to the dimensionality 1 . For any closed set $A$ the groups $B_{\nabla}^{00}(A)$ and $B_{\nabla}^{1}(R-A)$ are isomorphic.

8.2. Put $G=R-A$. We begin the proof of Theorems 8.11 and 8.12 by the consideration of the trivial case when $G$ consists of a finite number of points. As regards Theorem 8.12 in this case, from the connectivity of $R$ it follows that the number of (necessarily isolated) points, of which $G$ consists, cannot exceed 1 , so that we have either the case when $G$ consists of one point and $A$ is void, or the case when $G$ is void and $A=R$. Since in both these cases Theorem 8.12 is true, it is proved for a finite $G$.

Let us prove Theorem 8.11 under the assumption of a finite $G$. In this case $B_{\nabla}^{r+1}(G)=0$. But for $r>0$ the abstraction from the space $R$ of a finite 
number of isolated points does not, as may be easily seen, affect $B_{\Delta}^{r}(R)$, so that the group $B_{\nabla}^{r}(A)=B_{\nabla}^{r}(R-G)$ is isomorphic to the group $B_{\nabla}^{r}(R)$, which, by our assumptions, consists of the zero element only and hence is isomorphic to $B_{\nabla}^{r+1}(G)$. Thus for a finite $G$ Theorem 8.11 is also true.

Let now $G$ consist of an infinite number of points. In virtue of Theorems 7.54 and 7.6 for the proof of Theorems 8.11 and 8.12 it is sufficient to prove the following proposition:

8.21. For any natural number $r$ and any locally bicompact normal space $R$ the groups $B_{\nabla}^{r}(G)$ and $\lim \left[B_{\nabla}^{r}\left(K^{\alpha}-K_{1}^{\alpha}-C^{\alpha}\right) ; \pi_{\beta}^{\alpha}\right]$ are isomorphic.

\subsection{The proof of 8.2 .}

Definition 8.31. Let

$$
\Omega=\left\{e_{1}, \cdots, e_{p}, e_{p+1}, \cdots, e_{s}\right\}
$$

be a covering of the space $R$. Denote by $\phi_{\Omega}$ the sum of all those sets $\bar{e}_{i}$, which are bicompact and lie in $G$. The covering $\Omega$ is called regular with respect to $G$, if it satisfies the following two conditions:

$1^{\circ}$. No element of the first kind of the covering $\Omega$ meets $\phi_{\Omega}$.

$2^{\circ}$. The elements of the second kind of the covering $\Omega$ form a covering of the space $G$, which we denote by $G \Omega$ :

$$
G \Omega=\left\{e_{p+1}, \cdots, e_{s}\right\} .
$$

8.32. Every covering

$$
\Omega=\left\{e_{1}, \cdots, e_{p}, \cdots, e_{s}\right\}
$$

has a subdivision regular with respect to $G$.

Proof. Denote all non-void sets of the form $G \cap e_{i}$ by

$$
g_{1}, \cdots, g_{h} ;
$$

they form a covering $\Gamma$ of the set $G$. Denote by $\phi_{\Gamma}$ the sum of those among the the sets $\bar{g}_{i} \subset G$ which are bicompact. After this consider the sets $e_{i}-\phi_{\Gamma}$, $i=1,2, \cdots, p$, and denote them by

$$
e_{1}^{\prime}, \cdots, e_{p^{\prime}}^{\prime}
$$

The covering

$$
\Omega^{\prime}=\left\{e_{1}^{\prime}, \cdots, e_{p^{\prime}}^{\prime}, g_{1}, \cdots, g_{h}\right\}
$$

of the space $R$ is a subdivision of the covering $\Omega$. Since $\phi_{\Gamma}=\phi_{\Omega^{\prime}}, \Omega^{\prime}$ is regular with respect to $G$.

8.33. For every covering

$$
\Gamma=\left\{g_{1}, \cdots, g_{h}\right\}
$$


of the set $G$ there is a covering $\Omega$ of the space $R$ regular with respect to $G$ and such that

$$
G \Omega=\Gamma .
$$

In fact, denote by $\phi_{\Gamma}$ the sum of those of the sets $\bar{g}_{i}$ which are bicompact and lie in $G$. The covering $\Omega$ consisting of all elements of $\Gamma$ and of the set $R-\phi_{\Gamma}$ is the required covering.

Remark. Among the elements of the second kind of the covering $\Omega$ (regular with respect to $G$ ) those and only those are special elements of the covering $G \Omega$ which satisfy at least one of the following two conditions:

$1^{\circ}$. They are special elements in $\Omega$.

$2^{\circ}$. Their closure meets $A$.

8.4. Consider the system $\mathfrak{D}_{G}$ of all coverings

$$
\Gamma^{\alpha}=\left\{\begin{array}{c}
\alpha \\
g_{k}
\end{array}\right\}
$$$$
k=1,2, \cdots, h_{\alpha}
$$

of the set $G$. Denote by

$$
\Omega^{\alpha \lambda}=\left\{e_{i}^{\alpha \lambda}\right\} \cup\left\{g_{k}^{\alpha}\right\}, \quad i=1,2, \cdots, p_{\alpha \lambda}, k=1,2, \cdots, h_{\alpha},
$$

every regular with respect to $G$ covering of the space $R$ having $\Gamma^{\alpha}$ for the set of its elements of the second kind, i.e., satisfying the condition

$$
G \Omega^{\alpha \lambda}=\Gamma^{\alpha}
$$

For convenience we shall sometimes write instead of $g_{k}^{\alpha}$ also $e_{i}^{\alpha \lambda}$, where $i=p_{\alpha \lambda}+k$. The nerves of $\Omega^{\alpha \lambda}$ and $\Gamma^{\alpha}$ we denote respectively by $K^{\alpha \lambda}$ and $K^{\alpha}$; the special subcomplexes of $K^{\alpha \lambda}$ and $K^{\alpha}$ we denote respectively by $C^{\alpha \lambda}$ and $C^{\alpha}$. By $K_{1}^{\alpha \lambda}$ we denote, as usual, the subcomplex of $K^{\alpha \lambda}$ determined by those vertices $e_{i}^{\alpha \lambda} \varepsilon K^{\alpha \lambda}$ for which

$$
A \cap \bar{e}_{i}^{\alpha \lambda} \neq 0
$$

Let us prove the identity

$$
K^{\alpha}-C^{\alpha}=K^{\alpha \lambda}-K_{1}^{\alpha \lambda}-C^{\alpha \lambda}
$$

Let $t \varepsilon K^{\alpha}-C^{\alpha}$. The vertices of $t$ are some elements of the second kind $g_{k}^{\alpha} \varepsilon K^{\alpha} \varepsilon K^{\alpha \lambda} ;$ suppose they are $g_{1}^{\alpha}, g_{2}^{\alpha}, \cdots, g_{r}^{\alpha}$. We have

$$
g_{1}^{\alpha} \cap \ldots \cap g_{r}^{\alpha} \neq 0 .
$$

Since $t \notin C^{\alpha}$, among these $g_{k}^{\alpha}$ there is at least one, which is not a special element of the covering $\Gamma^{\alpha}$, i.e., has a bicompact closure lying in $G$; consequently $t$ can belong neither to $K_{1}^{\alpha \lambda}$ nor to $C^{\alpha \lambda}$, and so $t \varepsilon K^{\alpha \lambda}-K_{1}^{\alpha \lambda}-C^{\alpha \lambda}$. Conversely, if

$$
t \varepsilon K^{\alpha \lambda}-K_{1}^{\alpha \lambda}-C^{\alpha \lambda}
$$


then among the vertices of $t$ there is at least one belonging neither to $K_{1}^{\alpha \lambda}$ nor to $C^{\alpha \lambda}$, i.e., representing a certain $e_{i}^{\alpha \lambda}$ with a bicompact closure lying in $G$. In virtue of the regularity of $\Omega^{\alpha \lambda}$ with respect to $G$ all remaining vertices of $t$ are elements of the second kind of the covering $\Omega^{\alpha \lambda}$, i.e., elements of $\Gamma^{\alpha}$, so that $t \varepsilon K^{\alpha}$. Since among the vertices of $t$ at least one has a bicompact closure in $G, t \notin C^{\alpha}$, and, consequently, $t \varepsilon K^{\alpha}-C^{\alpha}$.

Since $K^{\alpha} \subset K^{\alpha \lambda}$, from 8.41 follows

$$
C^{\alpha} \subset K_{1}^{\alpha \lambda} \cup C^{\alpha \lambda}
$$

(which, by the way, follows also from the remark made at the end of 8.3).

From (8.41) and (8.42) it follows that the groups $L^{r}\left(K^{\alpha}-C^{\alpha}\right)$ and $L^{r}\left(K^{\alpha \lambda}-K_{1}^{\alpha \lambda}-C^{\alpha \lambda}\right)$ are isomorphic: a quite definite isomorphism between these groups is obtained if to every function $f^{r} \varepsilon L^{r}\left(K^{\alpha \lambda}-K_{1}^{\alpha \lambda}-C^{\alpha \lambda}\right)$ is correlated the function $G f^{r} \varepsilon L^{r}\left(K^{\alpha}-C^{\alpha}\right)$, where

$$
G f^{r}\left(t^{r}\right)=f^{r}\left(t^{r}\right) \quad \text { on all } t^{r} \varepsilon K^{\alpha} .
$$

It is easily seen that the isomorphism $G$ is commutative with the operator $\nabla$ :

$$
\nabla G f^{r}\left(t^{r+1}\right)=G \nabla f^{r}\left(t^{r+1}\right)
$$

for any $t^{r+1} \varepsilon K^{\alpha}$.

In fact, for any simplex $t^{r+1} \varepsilon K^{\alpha}$ we have

$$
\begin{gathered}
\nabla G f^{r}\left(t^{r+1}\right)=\sum_{t^{r+1}>t^{r}} G f^{r}\left(t^{r}\right)=\sum_{t^{r+1}>t^{r}} f^{r}\left(t^{r}\right)=\nabla f^{r}\left(t^{r}\right), \\
G \nabla f^{r}\left(t^{r+1}\right)=\nabla f^{r}\left(t^{r+1}\right) .
\end{gathered}
$$

From (8.43) it follows that the isomorphism $G$ between the groups $L^{r}\left(K^{\alpha \lambda}-K_{1}^{\alpha \lambda}-C^{\alpha \lambda}\right)$ and $L^{r}\left(K^{\alpha}-C^{\alpha}\right)$ generates an isomorphic mapping $\phi^{\alpha \lambda}$ of the group $B_{\nabla}^{r}\left(K^{\alpha \lambda}-K_{1}^{\alpha \lambda}-C^{\alpha \lambda}\right)$ on the group $B_{\nabla}^{r}\left(K^{\alpha}-C^{\alpha}\right)$.

8.5. Lemma 8.5. Any two coverings

$$
\Omega^{\alpha \lambda}=\left\{e_{i}^{\alpha \lambda}\right\} \cup\left\{g_{k}^{\alpha}\right\}, \quad \Omega^{\beta \mu}=\left\{e_{j}^{\beta \mu}\right\} \cup\left\{g_{l}^{\beta}\right\}
$$

regular with respect to $G$ have such a common subdivision

$$
\Omega^{\gamma \nu}=\left\{e_{f}^{\gamma \nu}\right\} \cup\left\{g_{m}^{\gamma}\right\}
$$

regular with respect to $G$ that every element of the second kind $g_{m}^{\gamma}$ of the covering $\Omega^{\gamma v}$ is contained in at least one element of the second kind of each of the coverings $\Omega^{\alpha \lambda}$ and $\Omega^{\beta \mu}$.

In fact, let

$$
\Omega=\left\{e_{1}, \cdots, e_{p}, g_{1}, \cdots, g_{h}\right\}
$$


be any common subdivision of the coverings $\Omega^{\alpha \lambda}$ and $\Omega^{\beta \lambda}$ regular with respect to $G$. Denote all non-void sets of the form $g_{k}^{\alpha} \cap g_{l}^{\beta} \cap g_{n}$ thus:

$$
g_{1}^{\gamma}, \cdots, g_{h_{\gamma}}^{\gamma}
$$

Since

$$
\cup_{g_{k}}^{\alpha}=\cup_{g_{l}}^{\beta}=\cup_{g_{n}}=G,
$$

we have also $\cup g_{m}^{\gamma}=G$. Denote by $\phi_{\gamma}$ the sum of all bicompact $\bar{g}^{\gamma} \subset G$ and put

$$
e_{i}^{\gamma \nu}=e_{i}-\phi_{\gamma}, \quad i=1,2, \cdots, p .
$$

For the covering

$$
\Omega^{\gamma \nu}=\left\{e_{1}^{\gamma \nu}, \cdots, e_{p}^{\gamma \nu}, g_{1}^{\gamma}, \cdots, g_{h_{\gamma}}^{\gamma}\right\}
$$

not only $\cup_{1 \leqq n \leqq h} g_{n}^{\gamma}=G$, but also $\phi_{\Omega^{\gamma \nu}}=\phi_{\gamma}$; so that $\Omega^{\gamma \nu}$ is regular with respect to $G$. Besides, for every $g_{m}^{\gamma}$ there are $g_{k}^{\alpha}, g^{\beta}$ and $g_{n}$ such that

$$
g_{m}^{\gamma}=g_{k}^{\alpha} \cap \underset{g_{l}^{\beta}}{\boldsymbol{\beta}} g_{n},
$$

and consequently

$$
g_{m}^{\gamma} \subset g_{k}^{\alpha}, \quad g_{m}^{\gamma} \subset g_{l}^{\beta} .
$$

The limit group of the spectrum

$$
\left[B_{\nabla}^{r}\left(K^{\alpha \lambda}-K_{1}^{\alpha \lambda}-C^{\alpha \lambda}\right) ; \pi_{\beta \mu}^{\alpha \lambda}\right]
$$

will not be changed if in this spectrum we retain only elements corresponding to coverings $\Omega^{\alpha \lambda}$ regular with respect to $G$ and put $\Omega^{\beta \lambda}>\Omega^{\alpha \lambda}$ only if $\Omega^{\beta \mu}$ follows after $\Omega^{\alpha \lambda}$ and every element of the second kind of $\Omega^{\beta \mu}$ is contained in some element of the second kind of $\Omega^{\alpha \lambda}$. then

Thus, if $B_{\nabla}^{r}\left(K^{\beta \mu}-K_{1}^{\beta \mu}-C^{\beta \mu}\right)>B_{\nabla}^{r}\left(K^{\alpha \lambda}-K_{1}^{\alpha \lambda}-C^{\alpha \lambda}\right)$ in the spectrum (8.51),

in the spectrum

$$
B_{\nabla}^{r}\left(K^{\beta}-C^{\beta}\right)>B_{\nabla}^{r}\left(K^{\alpha}-C^{\alpha}\right)
$$

$$
\left[B_{\nabla}^{r}\left(K^{\alpha}-C^{\alpha}\right) ; \pi_{\beta}^{\alpha}\right] .
$$

Let us, finally, prove that

$$
\stackrel{\beta \mu}{\beta \mu \lambda}{ }_{\beta}^{\alpha} \pi_{\beta \mu} u_{\alpha \lambda}=\underset{\pi_{\beta}}{\alpha} \phi_{\alpha}^{\alpha \lambda} u_{\alpha \lambda}
$$

for any element $u_{\alpha \lambda}$ of the group $B_{\nabla}^{r}\left(K^{\alpha \lambda}-K_{1}^{\alpha \lambda}-C^{\alpha \lambda}\right)$. To this end denote by $S_{\alpha \lambda}^{\beta \mu}$ some projection of $\Omega^{\beta \mu}$ into $\Omega^{\alpha \lambda}$ transforming every element of the second kind of $\Omega^{\beta \mu}$ into an element of the second kind of $\Omega^{\alpha \lambda}$. Such a projection $S_{\alpha \lambda}^{\beta \mu}$ generates a projection $S_{\alpha}^{\beta}$ of the covering $\Gamma^{\beta}$ into the covering $\Gamma^{\alpha}$. 
We denote the homomorphisms of the groups $L^{r}\left(K^{\alpha \lambda}-K_{1}^{\alpha \lambda}-C^{\alpha \lambda}\right)$ into $L^{r}\left(K^{\beta \mu}-K_{1}^{\beta \mu}-C^{\beta \mu}\right)$ and $L^{r}\left(K^{\alpha}-C^{\alpha}\right)$ into $L^{r}\left(K^{\beta}-C^{\beta}\right)$ generated by the projections $S_{\alpha \lambda}^{\beta \mu}$ and $S_{\alpha}^{\beta}$ respectively by $\sigma_{\beta \mu}^{\alpha \lambda}$ and $\sigma_{\beta}^{\alpha}$.

For the proof of (8.53) it is sufficient to show that for any element $f_{\alpha \lambda}^{\tau} \varepsilon L^{r}\left(K^{\alpha \lambda}-K_{1}^{\alpha \lambda}-C^{\alpha \lambda}\right)$ and any simplex $t_{\beta}^{r} \varepsilon K^{\beta} \subset K^{\beta \mu}$ we have

$$
G \sigma_{\beta \mu}^{\alpha \lambda} f_{\alpha \lambda}^{r}\left(t_{\beta}^{r}\right)=\sigma_{\beta}^{\alpha} G f_{\alpha \lambda}^{r}\left(t_{\beta}^{r}\right) .
$$

The last assertion follows from

$$
G \sigma_{\beta \mu}^{\alpha \lambda} f_{\alpha \lambda}^{r}\left(t_{\beta}^{r}\right)=\sigma_{\beta \mu}^{\alpha \lambda} f_{\alpha \lambda}^{r}\left(t_{\beta}^{r}\right)=f_{\alpha \lambda}^{r}\left(S_{\alpha \lambda}^{\beta \mu} t_{\beta}^{r}\right),
$$

if we take into account that for $t_{\beta}^{\tau} \varepsilon K^{\beta}$

$$
S_{\alpha}^{\beta} t_{\beta}^{r}=S_{\alpha \lambda}^{\beta \mu} t_{\beta} .
$$

Thus the spectra (8.51) and (8.52) satisfy all conditions of 3.61 and therefore their limit groups

$$
\lim \left[B_{\lambda}^{r}\left(K^{\alpha \lambda}-K_{1}^{\alpha \lambda}-C^{\alpha \lambda}\right) ; \pi_{\beta \mu}^{\alpha \lambda}\right], \quad \lim \left[B_{\nabla}^{r}\left(K^{\alpha}-C^{\alpha}\right) ; \stackrel{\pi_{\beta}^{\alpha}}{ }\right]
$$

are isomorphic. This proves 8.2 and, consequently, also the duality law of Kolmogoroff.

\section{ThE SECOND DEFINITION OF BETTI GROUPS}

9.1. Let $\mathfrak{O}$ be the system of all simple (open) coverings $\Omega^{\alpha}$ of a space $R$. As always, denote by $K^{\alpha}$ the nerve of the covering $\Omega^{\alpha}=\left\{e_{i}^{\alpha}\right\}$. Special elements of the covering $\Omega^{\alpha}$ we call those sets $e_{i}^{\alpha}$, the closure of which is not bicompact. The nerve of the aggregate of all special elements of the covering $\Omega^{\alpha}$ we denote by $C^{\alpha}$ and call it the special subcomplex of $K^{\alpha}$.

The barycentric subdivision of the complex $K^{\alpha}$ shall be denoted by $K^{1 \alpha}$, the barycentric subdivision of the complex $C^{\alpha}-$ by $C^{1 \alpha}$.

The vertices of the complex $K^{1 \alpha}$ are expressions of the form

$$
e_{i}^{1 \alpha}=e_{i_{0}}^{\alpha} \cdots e_{i_{r}}^{\alpha}, \quad \stackrel{\alpha}{e_{i k} \varepsilon \Omega^{\alpha},}
$$

(all $e_{i_{k}}^{\alpha}$ entering into $e_{t}^{1 \alpha}$ are different). An aggregate of certain vertices of the complex $K^{1 \alpha}$ defines a simplex of this complex if and only if for any two vertices

$$
\begin{aligned}
e_{i}^{1 \alpha} & =e_{i_{0}}^{\alpha} \cdots e_{i_{p}}^{\alpha}, \\
e_{j}^{1 \alpha} & =e_{j_{0}}^{\alpha} \cdots e_{j_{q}}^{\alpha}
\end{aligned}
$$

of this aggregate all factors in one of the two expressions (9.111) and (9.112), for instance, all factors $e_{j_{0}}^{\alpha}, \cdots, e_{j_{q}}^{\alpha}$ entering into the expression (9.112), enter also in the other expression, i.e., into (9.111). 
It is easily seen that the vertex

$$
e_{i}^{1 \alpha}=e_{i_{0}}^{\alpha} \cdots e_{i_{r}}^{\alpha}
$$

of the complex $K^{1 \alpha}$ belongs to the complex $C^{1 \alpha}$ (is a special vertex of $K^{1 \alpha}$ ) if and only if all elements $e_{i_{0}}^{\alpha}, \cdots, e_{\imath_{r}}^{\alpha}$ are special elements. We introduce yet the following notation. For any vertex $e_{i}^{1 \alpha}=e_{i_{0}}^{\alpha} \cdots e_{i_{r}}^{\alpha} \varepsilon K^{1 \alpha}$ denote by $\left|e_{i}^{1 \alpha}\right|=\left|e_{i_{0}}^{\alpha} \cdots e_{i_{r}}^{\alpha}\right|$ the set

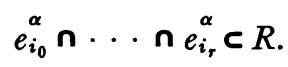

9.11. Every projection $S_{\alpha}^{\beta}$ of the complex $K^{\beta}$ into $K^{\alpha}$ generates a simplicial mapping $S_{1 \alpha}^{1 \beta}$ of the complex $K^{1 \beta}$ into the complex $K^{1 \alpha}$; moreover, we have

$$
S_{1 \alpha}^{1 \beta}\left(C^{1 \beta}\right) \subset C^{1 \alpha} \text {. }
$$

We obtain this mapping correlating to the centre of gravity of any simplex $t_{\beta} \varepsilon K^{\beta}$ the centre of gravity of its image $S_{\alpha}^{\beta} t_{\beta}$ and observing that $S_{\alpha}^{\beta} C^{\beta} \subset C^{\alpha}$.

9.2. Definition 9.20. A covering $\Omega^{\alpha}$ is called multiplicative if the intersection of any number of elements of $\Omega^{\alpha}$ is either void or an element of $\Omega^{\alpha}$.

9.21. Every covering $\Omega^{\alpha}$ has a subdivision $\Omega^{\beta}$, which is a multiplicative covering.

For $\Omega^{\beta}$ it is sufficient to take the covering consisting of all elements of $\Omega^{\alpha}$ and of all non-void sets, which are intersections of several elements of $\Omega^{\alpha}$.

DeFINITION 9.22. By a barycentric subdivision of the covering $\Omega^{\alpha}$ we mean a complex $K^{2 \alpha}$, whose vertices are elements $e_{i}^{\alpha}$ of the covering $\Omega^{\alpha}$ and whose simplexes are decreasing sequences

$$
e_{i_{0}}^{\alpha} \supset e_{i_{1}}^{\alpha} \supset \cdots \supset e_{i_{r}}^{\alpha}
$$$$
e_{i k} \neq e_{i_{k+1}} \text {, }
$$

of elements of the covering $\Omega^{\alpha}$.

The complex $K^{2 \alpha}$ is evidently a subcomplex of the complex $K^{\alpha}$. Put $C^{2 \alpha}=K^{2 \alpha} \cap C^{\alpha}$; we call $C^{2 \alpha}$ the special subcomplex of the complex $K^{2 \alpha}$.

Let us come to an agreement, which will enable us to consider $K^{2 \alpha}$ also as a subcomplex of the complex $K^{1 \alpha}$. To this end observe in the first place the following: if for the vertices of the complex $K^{1 \alpha}$

$$
e_{i}^{1 \alpha}=e_{i_{0}}^{\alpha} \cdots e_{i p}^{\alpha}, \quad e_{j}^{1 \alpha}=e_{j_{0}}^{\alpha} \cdots e_{j_{q}}^{\alpha}
$$

we have $\left|e_{i}^{1 \alpha}\right|=\left|e_{j}^{1 \alpha}\right|$, then, obviously, for $e_{\mathbf{k}}^{1 \alpha}$ consisting of all different factors entering into $e_{i}^{1 \alpha}$ or into $e_{j}^{1 \alpha}$ we shall have

$$
\left|e_{k}^{1 \alpha}\right|=\left|e_{i}^{1 \alpha}\right|=\left|e_{j}^{1 \alpha}\right|
$$

Therefore, among all expressions 


$$
e_{i}^{1 \alpha}=e_{i_{0}}^{\alpha} \cdots e_{i_{r}}^{\alpha}
$$

with one and the same set $\left|e_{i}^{1 \alpha}\right|$ we shall have one longest, i.e., one consisting of the maximum number of factors. Correlating to every vertex $e_{i}^{2 \alpha}$ of the complex $K^{2 \alpha}$ the longest expression $e_{i}^{1 \alpha}$ satisfying the condition $\left|e_{i}^{1 \alpha}\right|=e_{i}^{2 \alpha}$ and observing that from $e_{i}^{2 \alpha} \supset e_{j}^{2 \alpha}$ follows that all factors of $e_{j}^{1 \alpha}$ are contained among the factors of $e_{i}^{1 \alpha}$, we obtain an isomorphic mapping of the complex $K_{i}^{2 \alpha}$ into the complex $K_{i}^{1 \alpha}$. If we now identify $e_{i}^{2 \alpha}$ with $e_{i}^{1 \alpha}$, then we can consider $K^{2 \alpha}$ as a subcomplex of the complex $K^{1 \alpha}$.

On the other hand, correlating to every vertex $e_{i}^{1 \alpha}$ of the complex $K^{1 \alpha}$ the vertex $\left|e_{i}^{1 \alpha}\right|$ of the complex $K^{2 \alpha}$, we obtain a simplicial mapping $S_{2 \alpha}^{1 \alpha}$ of the complex $K^{1 \alpha}$ on $K^{2 \alpha}$, which, if $K^{2 \alpha}$ is considered as a subcomplex of the complex $K^{1 \alpha}$, leaves all simplexes of the complex $K^{2 \alpha}$ fixed. Besides, $S_{2 \alpha}^{1 \alpha}\left(C^{1 \alpha}\right) \subset C^{2 \alpha}$ and hence:

9.23. The mapping $S_{2 \alpha}^{1 \alpha}$ of the complex $K^{1 \alpha}$ on the complex $K^{2 \alpha}$ generates an isomorphic mapping $\rho_{2 \alpha}^{1 \alpha}$ of the group $B_{\nabla}^{r}\left(K^{1 \alpha} \bmod C^{1 \alpha}\right)$ on the group $B_{\nabla}^{r}\left(K^{2 \alpha} \bmod C^{2 \alpha}\right)$ and an isomorphic mapping $\sigma_{1 \alpha}^{2 \alpha}$ of the group $B_{\nabla}^{r}\left(K^{2 \alpha}-C^{2 \alpha}\right)$ on the group $B_{\nabla}^{r}\left(K^{1 \alpha}-C^{1 \alpha}\right)$.

Correlating to every vertex

$$
e_{i}^{1 \alpha}=\stackrel{\alpha}{e_{i_{0}}} \cdots e_{i_{r} \varepsilon}^{\alpha} K^{1 \alpha}
$$

one of the vertices of its bearer in the complex $K^{\alpha}$ (i.e., one of the $e_{i_{h}}^{\alpha}$ ), we obtain a simplicial mapping $S_{\alpha}^{1 \alpha}$ of the complex $K^{1 \alpha}$ on the complex $K^{\alpha}$ generating, as we know, an isomorphic mapping $\rho_{\alpha}^{1 \alpha}$ of the group $B_{\Delta}^{r}\left(K^{1 \alpha} \bmod C^{1 \alpha}\right)$ on the group $B_{\Delta}^{r}\left(K^{\alpha} \bmod C^{\alpha}\right)$ and, consequently, an isomorphic mapping $\sigma_{1 \alpha}^{\alpha}$ of the group $B_{\Delta}^{r}\left(K^{\alpha}-C^{\alpha}\right)$ on the group $B_{\nabla}^{r}\left(K^{1 \alpha}-C^{1 \alpha}\right)$.

9.3. Let there be given two multiplicative coverings $\Omega^{\alpha}$ and $\Omega^{\beta}$ of the space $R$, of which $\Omega^{\beta}$ follows after $\Omega^{\alpha}$. Construct the projection $S_{\alpha}^{\beta}$ of the covering $\Omega^{\beta}$ into the covering $\Omega^{\alpha}$ in the following way: for every $e_{j}^{\beta} \varepsilon \Omega^{\beta}$ we take for $S_{\alpha}^{\beta} e_{j}^{\beta}$ the smallest $e_{i}^{\alpha}$ containing $e_{j}^{\beta}$, i.e., the intersection of all $e_{i}^{\alpha}$ containing the given $e_{j}^{\beta}$. The so-constructed projection is called the canonical projection of the covering $\Omega^{\beta}$ into the multiplicative covering $\Omega^{\alpha}$.

9.31. Under the canonical projection $S_{\alpha}^{\beta}$ of the multiplicative covering $\Omega^{\beta}$ into the multiplicative covering $\Omega^{\alpha}$ the complex $K^{2 \beta} \subset K^{\beta}$ is mapped into $K^{2 \alpha} \subset K^{\alpha}$ so that $C^{2 \beta}$ is mapped into $C^{2 \alpha}$.

The second assertion follows from the first, since $C^{2 \alpha}=K^{2 \alpha} \cap C^{\alpha}$, $C^{2 \beta}=K^{2 \beta} \cap C^{\beta}$ and $S_{\alpha}^{\beta} C^{\beta} \subset C^{\alpha}$.

For the proof of the first assertion of 9.31 it is sufficient to show

9.311. If $S_{\alpha}^{\beta}$ is a canonical projection of $\Omega^{\beta}$ into $\Omega^{\alpha}$ and if $e_{i}^{\beta} \supset e_{j}^{\beta}$, then $S_{\alpha}^{\beta} e_{i}^{\beta} \supset S_{\alpha}^{\beta} e_{j}^{\beta}$. 
In fact,

$$
e_{j}^{\beta} \subset S_{\alpha}^{\beta} e_{j}^{\beta}, \quad e_{j}^{\beta} \subset e_{i}^{\beta} \subset S_{\alpha}^{\beta} e_{i}^{\beta} .
$$

Since $S_{\alpha}^{\beta} e_{j}^{\beta}$ is the smallest element of the covering $\Omega^{\alpha}$ containing $e_{j}^{\beta}$,

$$
S_{\alpha}^{\beta} e_{i}^{\beta} \cap S_{\alpha}^{\beta} e_{j}^{\beta}=S_{\alpha}^{\beta} e_{j}^{\beta},
$$

i.e.,

q.e.d.

$$
S_{\alpha}^{\beta} e_{i}^{\beta} \supset S_{\alpha}^{\beta} e_{j}^{\beta}
$$

Thus every canonical projection $S_{\alpha}^{\beta}$ generates a homomorphic mapping $\tilde{\omega}_{2 \alpha}^{2 \beta}$ of the group $B_{\Delta}^{r}\left(\dot{K}^{2 \beta} \bmod C^{2 \beta}\right)$ into the group $B_{\Delta}^{r}\left(K^{2 \alpha} \bmod C^{2 \alpha}\right)$ and a homomorphic mapping $\pi_{2 \beta}^{2 \alpha}$ of the group $B_{\nabla}^{r}\left(K^{2 \alpha}-C^{2 \alpha}\right)$ into the group $B_{\nabla}^{r}\left(K^{2 \beta}-C^{2 \beta}\right)$.

On ground of 9.11 we know, moreover, that $S_{\alpha}^{\beta}$ generates through the simplicial mapping $S_{\alpha 1}^{\beta 1}$ a homomorphic mapping $\tilde{\omega}_{\alpha 1}^{\beta 1}$ of the group $B_{\Delta}^{r}\left(K^{1 \beta} \bmod C^{1 \beta}\right)$ into the group $B_{\Delta}^{r}\left(K^{1 \alpha} \bmod C^{1 \alpha}\right)$ and a homomorphic mapping $\pi_{\beta 1}^{\alpha 1}$ of the group $B_{\nabla}^{r}\left(K^{1 \alpha}-C^{1 \alpha}\right)$ in to the group $B_{\nabla}^{r}\left(K^{1 \beta}-C^{1 \beta}\right)$.

Let us now prove the formulae

$$
\begin{aligned}
\tilde{\omega}_{1 \alpha}^{1 \beta} & =\left(\rho_{\alpha}^{1 \alpha}\right)^{-1} \tilde{\omega}_{\alpha}^{\beta} \rho_{\beta}^{1 \beta}, \\
\tilde{\omega}_{2 \alpha}^{2 \beta} & =\rho_{2 \alpha} \tilde{\omega}_{1 \alpha}^{1 \beta}\left(\rho_{2 \beta}^{1 \beta}\right)^{-1}, \\
1 \alpha & =\sigma_{1 \beta}^{\beta} \pi_{\beta}^{\alpha}\left(\sigma_{1 \alpha}^{\alpha}\right)^{-1}, \\
\pi_{1 \beta} & 2 \alpha \\
\pi_{2 \beta} & =\left(\sigma_{1 \beta}^{2 \beta}\right)^{-1} \pi_{1 \beta}^{1 \alpha} \sigma_{1 \alpha}^{2 \alpha} .
\end{aligned}
$$

On ground of Theorem III (see the Addendum) it is sufficient to prove the formulae (9.32) which may be written in the form

$$
\begin{gathered}
\rho_{\alpha}^{1 \alpha} \tilde{\omega}_{1 \alpha}^{1 \beta}=\tilde{\omega}_{\alpha \rho_{\beta}^{1 \beta}}^{\beta}, \\
\tilde{\omega}_{2 \alpha}^{2 \beta} \rho_{2 \beta}=\rho_{2 \alpha}^{1 \alpha} \tilde{\omega}_{1 \alpha}^{1 \beta} .
\end{gathered}
$$

Observe that the homomorphism $\rho_{\alpha}^{1 \alpha} \tilde{\omega}_{1 \alpha}^{1 \beta}$ is generated by the simplicial mapping $S_{\alpha}^{1 \alpha} S_{1 \alpha}^{1 \beta}$ of the complex $K^{1 \beta}$ into the complex $K^{\alpha}$; in the same way the homomorphism $\tilde{\omega}_{\alpha}^{\beta} \rho_{\beta}^{1 \beta}$ is generated by the simplicial mapping $S_{\alpha}^{\beta} S_{\beta}^{1 \beta}$ of the complex $K^{1 \beta}$ into the complex $K^{\alpha}$. Hence for the proof of (9.341) it is sufficient to show the truth of the following assertion:

9.32. For any simplex $t_{1 \beta} \varepsilon K^{1 \beta}$ the simplexes $S_{\alpha}^{1 \alpha} S_{1 \alpha}^{1 \beta} t_{1 \beta}$ and $S_{a}^{\beta} S_{\beta}^{1 \beta} t_{1 \beta}$ are faces of a.certain simplex $T_{\alpha} \varepsilon K^{\alpha}$; moreover, if $t_{1 \beta} \varepsilon C^{1 \beta}$, we may take $T_{\alpha} \varepsilon C^{\alpha}$.

In the same way (9.342) follows from

9.33. For any simplex $t_{1 \beta} \varepsilon K^{1 \beta}$ the simplexes $S_{2 \alpha}^{1 \alpha} S_{1 \alpha}^{1 \beta} t_{1 \beta}$ and $S_{2 \alpha}^{2 \beta} S_{2 \beta}^{1 \beta} t_{1 \beta}$ are faces of a certain simplex $T_{\alpha} \varepsilon K^{\alpha}$; moreover, if $t_{1 \beta} \varepsilon C^{1 \beta}$, we may take $T_{\alpha} \varepsilon C^{\alpha}$. 
Proof. Let

$$
t_{1 \beta}=e_{0}^{1 \beta} \cdots e_{r}^{1 \beta} ; \quad e_{\mu}^{1 \beta}=e_{\mu_{0}}^{\beta} \cdots e_{\mu_{q}}^{\beta},
$$

where $q=q(\mu)$ depends on $\mu$,

$$
\begin{gathered}
S_{\beta}^{1 \beta} e_{\mu}^{1 \beta}=e_{\mu_{0}}^{\beta}, \\
S_{\alpha}^{\beta} e_{\mu_{0}}^{\beta}=e_{i}^{\alpha} \supset e_{\mu_{0}}^{\beta} \supset\left|e_{\mu}^{1 \beta}\right|,
\end{gathered}
$$

so that

$$
S_{\alpha}^{\beta} S_{\beta}^{1 \beta} e_{\mu}^{1 \beta} \supset\left|e_{\mu}^{1 \beta}\right|
$$

If $e_{\mu}^{1 \beta} \varepsilon C^{1 \beta}$, then $e_{\mu_{0}}^{\beta} \varepsilon C^{\beta}$ and $e_{i}^{\alpha} \varepsilon C^{\alpha}$.

Let

$$
S_{1 \alpha}^{1 \beta} e_{\mu}^{1 \beta}=e_{\lambda}^{1 \alpha}=e_{\lambda_{0}}^{\alpha} \cdots e_{\lambda_{p}}^{\alpha}, \quad S_{\alpha}^{1 \alpha} e_{\lambda}^{1 \alpha}=e_{\alpha}^{\alpha} .
$$

If $e_{\mu}^{1 \beta} \varepsilon C^{1 \beta}$, then $e_{\lambda}^{1 \alpha} \varepsilon C^{1 \alpha}, e_{\kappa}^{\alpha} \varepsilon C^{\alpha}$.

Since

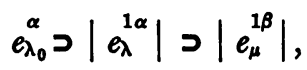

we have

$$
S_{\alpha}^{1 \alpha} S_{1 \alpha}^{1 \beta} e_{\mu}^{1 \beta} \supset\left|e_{\mu}^{1 \beta}\right|
$$

whence

$$
\bigcap_{0 \leqq \mu \leqq r} S_{\alpha}^{\beta} S_{\beta}^{1 \beta} e_{\mu}^{1 \beta} \cap S_{\alpha}^{1 \alpha} S_{1 \alpha}^{1 \beta} e_{\mu}^{1 \beta} \supset \bigcap_{0 \leqq \mu \leqq r}\left|e_{\mu}^{1 \beta}\right| \neq 0,
$$

i.e., all $S_{\alpha}^{\beta} S_{\beta}^{1 \beta} e_{\mu}^{1 \beta}$ and $S_{\alpha}^{1 \alpha} S_{1 \alpha}^{1 \beta} e_{\mu}^{1 \beta}$ are vertices of a certain simplex $T_{\alpha} \varepsilon K^{\alpha}$. If all $e_{\mu}^{1 \beta} \varepsilon C^{1 \beta}$, then both $S_{\alpha}^{\beta} S_{\beta}^{1 \beta} e_{\mu}^{1 \beta}$ and $S_{\alpha}^{1 \alpha} S_{1 \alpha}^{1 \beta} e_{\mu}^{1 \beta}$ are vertices of $C^{\alpha}$ and hence $T_{\alpha} \varepsilon C^{\alpha}$.

The assertion 9.32 is thus proved.

The assertion 9.33 is proved in the same way. In fact, in the first place we have

$$
S_{2 \beta}^{1 \beta} e_{\mu}^{1 \beta}=\left|e_{\mu}^{1 \beta}\right|=e_{\nu}^{2 \beta}, \quad S_{2 \alpha}^{2 \beta} e_{\nu}^{2 \beta}=e_{\lambda}^{2 \alpha} \supset\left|e_{\mu}^{1 \beta}\right|
$$

and if $e_{\mu}^{1 \beta} \varepsilon C^{1 \beta}$, then $e_{\lambda}^{2 \alpha} \varepsilon C^{2 \alpha}$.

Thus

$$
S_{2 \alpha}^{2 \beta} S_{2 \beta}^{1 \beta} e_{\mu}^{1 \beta} \supset\left|e_{\mu}^{1 \beta}\right|
$$

Let

$$
S_{1 \alpha}^{1 \beta} e_{\mu}^{1 \beta}=e_{k}^{1 \alpha}=e_{x_{0}}^{\alpha} \cdots e_{x_{n}}^{\alpha} .
$$

Again for $e_{\mu}^{1 \beta} \varepsilon C^{1 \beta}$ we have $e_{\alpha}^{1 \alpha} \varepsilon C^{1 \alpha}$, 


$$
\begin{gathered}
S_{2 \alpha}^{1 \alpha} e_{\alpha}^{1 \alpha}=e_{\eta}^{2 \alpha}=\left|e_{\alpha}^{1 \alpha}\right| \supset\left|e_{\mu}^{1 \beta}\right| \\
S_{2 \alpha}^{1 \alpha} S_{1 \alpha}^{1 \beta} e_{\mu}^{1 \beta} \supset\left|e_{\mu}^{1 \beta}\right|
\end{gathered}
$$

and if $e_{\mu}^{1 \beta} \varepsilon C^{1 \beta}$, then $e^{2 \alpha} \varepsilon C^{2 \alpha}$.

From the inclusions (9.391) and (9.392) we easily deduce (9.33).

From the formulae (9.32) and (9.33) follow

$$
\begin{array}{ll}
\tilde{\omega}_{1 \alpha}^{1 \beta} \tilde{\omega}_{1 \beta}^{1 \gamma}=\tilde{\omega}_{1 \alpha}^{1 \gamma}, & \tilde{\omega}_{2 \alpha}^{2 \beta} \tilde{\omega}_{2 \beta}^{2 \gamma}=\tilde{\omega}_{2 \alpha}^{2 \gamma}, \\
\pi_{1 \gamma}^{1 \beta} \pi_{1 \beta}=\pi_{1 \gamma}^{1 \alpha}, & \pi_{2 \gamma}^{2 \beta} \pi_{2 \beta}^{2 \alpha}=\pi_{2 \gamma}^{2 \alpha} .
\end{array}
$$

Thus we have the inverse and the direct spectra:

$$
\begin{aligned}
{\left[B_{\Delta}^{r}\left(K^{1 \alpha} \bmod C^{1 \alpha}\right) ; \tilde{\omega}_{1 \alpha}^{1 \beta}\right], } & {\left[B_{\Delta}^{r}\left(K^{2 \alpha} \bmod C^{2 \alpha}\right) ; \tilde{\omega}_{2 \alpha}^{2 \beta}\right], } \\
{\left[B_{\nabla}^{r}\left(K^{1 \alpha}-C^{1 \alpha}\right) ; \pi_{1 \beta}^{1 \alpha}\right], } & {\left[B_{\nabla}^{r}\left(K^{2 \alpha}-C^{2 \alpha}\right) ; \pi_{2 \beta}^{2 \alpha}\right], }
\end{aligned}
$$

the limit groups of which are, on the basis of 3.41 and the formulae (9.32), (9.33), isomorphic to the groups $B_{\Delta}^{r}(R)$ and $B_{\nabla}^{r}(R)$ respectively.

9.4. On the basis of what has been proved we may now formulate the following new definition of the (inner) Betti groups of a space $R$.

Consider the system $\mathfrak{O}$ of all finite simple multiplicative coverings

$$
\Omega^{\alpha}=\left\{e_{i}^{\alpha}\right\}, \quad i=1,2, \cdots, s_{\alpha},
$$

of the space $R$, whose elements are open sets. Consider the functions

$$
f_{\alpha}^{r}=f_{\alpha}^{r}\left(e_{i_{0}}^{\alpha}, \cdots, e_{i_{r}}^{\alpha}\right)
$$

with values from a given commutative group $J$ defined for all possible decreasing sequences

$$
\stackrel{\alpha}{e_{i_{0}}} \supset \underset{e_{i_{1}}^{\alpha}}{\alpha} \supset \cdots \supset e_{i_{r}}^{\alpha}
$$

consisting of $r+1$ elements of the covering $\Omega^{\alpha}$. By assumption $f_{\alpha}^{r}\left(e_{i_{0}}^{\alpha}, \cdots, e_{i_{r}}^{\alpha}\right)$ may be different from zero only when the sequence

$$
e_{i_{0}}^{\alpha} \supset e_{i_{1}}^{\alpha} \supset \cdots \supset e_{i_{r}}^{\alpha}
$$

is strictly decreasing (i.e., when $e_{i_{k}}^{\alpha} \neq e_{i_{k+1}}^{\alpha}$ for $\kappa=0,1, \cdots, r-1$ ). The functions $f_{\alpha}^{r}$ form the group $L^{r}\left(\Omega^{\alpha}, J\right)$. The boundary operators are defined thus:

The lower:

$$
\Delta f_{\alpha}^{r}\left(e_{i_{0}}^{\alpha}, \cdots, e_{i_{r-1}}^{\alpha}\right)=\sum_{i}(-1)^{\alpha} f_{\alpha}^{r}\left(e_{i_{0}}^{\alpha}, \cdots, e_{i_{\kappa-1}}^{\alpha}, e_{i}^{\alpha}, e_{i_{\kappa}}^{\alpha}, \cdots, e_{i_{r-1}}^{\alpha}\right)
$$

(where the summation is extended over all $e_{i}^{\alpha}$ for which there exists such an $e_{i_{\kappa}}^{\alpha}$ that $\left.e_{i_{k-1}}^{\alpha} \supset e_{i}^{\alpha} \supset e_{i_{k}}^{\alpha}\right)$.

The upper: 


$$
\nabla f_{\alpha}^{r}\left(e_{i_{0}}^{\alpha}, \cdots, e_{i_{r+1}}^{\alpha}\right)=\sum_{\alpha}(-1)^{\alpha} f_{\alpha}^{r}\left(e_{i_{0}}^{\alpha}, \cdots, e_{i_{\kappa-1}}^{\alpha}, e_{i_{\alpha+1}}^{\alpha}, \cdots, e_{i_{r+1}}^{\alpha}\right) .
$$

(Remark. It is easily seen that applying the operators $\Delta$ and $\nabla$ to functions satisfying the condition to which the functions $f_{\alpha}^{r}$ were just subjected, we obtain functions satisfying the same condition (i.e., vanishing for all sequences $e_{i_{0}}^{\alpha} \supset \ldots \supset e_{t_{r}}^{\alpha}$ containing equal elements). This assertion is obvious for the operator $\Delta$. Let us prove it for the operator $\nabla$. If in the sequence $e_{i_{0}}^{\alpha} \supset \cdots \supset e_{i_{t+1}}^{\alpha}$ there is more than one pair of coinciding elements, then in each of the sequences $e_{i_{0}}^{\alpha} \supset \cdots \supset e_{i_{k-1}}^{\alpha} \supset e_{i_{k+1}}^{\alpha} \supset \cdots \supset e_{i_{r+1}}^{\alpha}$ there is at least one pair of coinciding elements; but then the left-hand side, and consequently the righthand side of the equality (9.4) vanishes. If, on the other hand, in the sequence $e_{i_{0}}^{\alpha} \supset \cdots \supset e_{t_{r+1}}^{\alpha}$ there is only one pair of coinciding elements, say

$$
e_{i_{\kappa}}^{\alpha}=e_{i_{\kappa+1}}^{\alpha}
$$

then on the right-hand side of the equality (9.4) only two terms can be different from zero, namely

$$
\begin{aligned}
& (-1)^{\kappa} f_{\alpha}^{r}\left(e_{i_{0}}^{\alpha}, \cdots, e_{i_{\kappa-1}}^{\alpha}, e_{i_{\kappa+1}}^{\alpha}, e_{i_{\kappa+2}}^{\alpha}, \cdots, e_{i_{r+1}}^{\alpha}\right), \\
& (-1)^{\alpha+1} f_{\alpha}^{r}\left(e_{i_{0}}^{\alpha}, \cdots, e_{i_{\kappa-1}}^{\alpha}, e_{i_{\kappa}}^{\alpha}, e_{i_{\kappa+2}}^{\alpha}, \cdots, e_{i_{r+1}}^{\alpha}\right) ;
\end{aligned}
$$

but these terms differ only in the sign and their sum is zero, so that again both sides of the equality (9.4) vanish.)

Let, as always, $X$ and $\Xi$ be two groups dual to each other, of which $X$ is discrete and $\Xi$ bicompact. In the group $L^{r}\left(\Omega^{\alpha}, \Xi\right)$ we consider the following subgroups: The subgroup of relative cycles $Z_{\Delta}^{r}\left(\Omega^{\alpha}, \Xi\right)$ consisting of all functions $f_{\alpha}^{r}$ satisfying the following condition: if $e_{i_{0}}^{\alpha} \supset \cdots \supset e_{\iota_{T}}^{\alpha}$ and $\Delta f_{\alpha}^{r}\left(e_{i_{0}}^{\alpha}, \cdots, e_{\iota_{r}}^{\alpha}\right) \neq 0$, then $\bar{e}_{i}^{\alpha}$ is not bicompact. In the group $Z_{\Delta}^{r}\left(\Omega^{\alpha}, \Xi\right)$ we consider the subgroup $H_{\Delta}^{r}\left(\Omega^{\alpha}, \Xi\right)$ of relative cycles homologous to zero: by definition $H_{\Delta}^{r}\left(\Omega^{\alpha}, \Xi\right)$ consists of functions $f_{\alpha}^{r}$ satisfying the following condition: there exists such a function $f_{\alpha}^{r+1} \varepsilon L^{r+1}\left(\Omega^{\alpha}, \Xi\right)$ that the function $\Delta f_{\alpha}^{r+1}-f_{\alpha}^{r}$ vanishes for all $e_{i_{0}}^{\alpha} \supset \ldots \supset e_{t_{r}}^{\alpha}$ with bicompact $\bar{e}_{t_{t^{\prime}}}^{\alpha}$.

In the group $L^{r}\left(\Omega^{\alpha}, X\right)$ we consider the subgroup $L_{0}^{r}\left(\Omega^{\alpha}, X\right)$ consisting of all functions $f_{\alpha}^{r}$ satisfying the following condition: if

$$
e_{i_{0}}^{\alpha} \supset \cdots \supset e_{i_{r}}^{\alpha}
$$

and $\bar{e}_{i_{r}}^{\alpha}$ is not bicompact, then

$$
f_{\alpha}^{r}\left(e_{i_{0}}^{\alpha}, \cdots, e_{i_{r}}^{\alpha}\right)=0 .
$$

Further we consider the group $Z_{\nabla}^{\tau}\left(\Omega^{\alpha}, X\right)$ of all $\nabla$-cycles contained in $L_{0}^{r}\left(\Omega^{\alpha}, X\right)$, i.e., of all functions $f_{\alpha}^{r} \varepsilon L_{0}^{r}\left(\Omega^{\alpha}, X\right)$ satisfying the condition $\nabla f_{\alpha}^{r}=0$. In the group $Z_{\nabla}^{r}\left(\Omega^{\alpha}, X\right)$ we consider in its turn the subgroup $H_{\nabla}^{r}\left(\Omega^{\alpha}, X\right)$ of 
cycles homologous to zero, i.e., the subgroup consisting of functions $f_{\alpha}^{r}$ for which there exist such functions $f_{\alpha}^{r-1} \varepsilon L_{0}^{r}\left(\Omega^{\alpha}, X\right)$ that $\Delta f_{\alpha}^{r-1}=f_{\alpha}^{r}$.

(Remark. In the case of a bicompact $R$ all these definitions may be simplified: $Z_{\Delta}^{r}\left(\Omega^{\alpha}, \Xi\right)$ becomes the group of all cycles, i.e., functions $f_{\alpha}^{r} \varepsilon L^{r}\left(\Omega^{\alpha}, \Xi\right)$ satisfying the condition $\Delta f_{\alpha}^{r}=0 ; H_{\Delta}^{r}\left(\Omega^{\alpha}, X\right)$ becomes the subgroup of all cycles homologous to zero, i.e., the subgroup of those functions $f_{\alpha}^{r} \varepsilon L^{r}\left(\Omega^{\alpha}, \Xi\right)$ for which there exist $f_{\alpha}^{r+1}$ with $\Delta f_{\alpha}^{r+1}=f_{\alpha}^{r}$; the group $L_{0}^{r}\left(\Omega^{\alpha}, X\right)$ coincides with $L^{r}\left(\Omega^{\alpha}, X\right)$; the group $Z_{\nabla}^{\tau}\left(\Omega^{\alpha}, X\right)$ is the group of all $\nabla$-cycles, i.e., of all functions $f_{\alpha}^{r}$ with $\nabla f_{\alpha}^{r}=0 ; H_{\nabla}^{r}\left(\Omega^{\alpha}, X\right)$ is the group of all $\nabla$-cycles homologous to zero, i.e., of those functions $f_{\alpha}^{r}$ for which there exist $f_{\alpha}^{r-1}$ with $\Delta f_{\alpha}^{r-1}=f_{\alpha}^{r}$.)

The factor groups

$$
\begin{aligned}
& B_{\Delta}^{r}\left(\Omega^{\alpha}, J\right)=Z_{\Delta}^{r}\left(\Omega^{\alpha}, J\right)-H_{\Delta}^{r}\left(\Omega^{\alpha}, J\right), \\
& B_{\nabla}^{r}\left(\Omega^{\alpha}, J\right)=Z_{\nabla}^{r}\left(\Omega^{\alpha}, J\right)-H_{\nabla}^{r}\left(\Omega^{\alpha}, J\right)
\end{aligned}
$$

are called, respectively, the Betti $\Delta$-and $\nabla$-groups of the covering $\Omega^{\alpha}$.

For a bicompact $R$ and $r=0$ we define, besides, the groups $Z_{\Delta}^{00}\left(\Omega^{\alpha}, J\right)$ and $Z_{\nabla}^{00}\left(\Omega^{\alpha}, J\right)$. The group $Z_{\Delta}^{00}\left(\Omega^{\alpha}, J\right)$ is the group of all functions $f_{\alpha}^{r}$, the sum of values of which is equal to zero; the group $Z_{\nabla}^{00}\left(\Omega^{\alpha}, J\right)$ is the group of all constant functions (observe that the group $Z_{\nabla}^{0}\left(\Omega^{\alpha}, J\right)$ is the group of all functions constant on every component of the covering $\Omega^{\alpha}$ ).

The groups $B_{\Delta}^{00}\left(\Omega^{\alpha}, J\right)$ and $B_{\nabla}^{00}\left(\Omega^{\alpha}, J\right)$ we define by the equalities

$$
\begin{aligned}
& B_{\Delta}^{00}\left(\Omega^{\alpha}, J\right)=Z_{\Delta}^{00}\left(\Omega^{\alpha}, J\right)-H_{\Delta}^{0}\left(\Omega^{\alpha}, J\right), \\
& B_{\nabla}^{00}\left(\Omega^{\alpha}, J\right)=Z_{\nabla}^{0}\left(\Omega^{\alpha}, J\right)-Z_{\nabla}^{00}\left(\Omega^{\alpha}, J\right) .
\end{aligned}
$$

If the covering $\Omega^{\beta}$ is a subdivision of the covering $\Omega^{\alpha}$, then we make correspond to every element of the covering $\Omega^{\beta}$ the smallest element of the covering $\Omega^{\alpha}$ containing it. The so-obtained mappings of the covering $\Omega^{\beta}$ into the covering $\Omega^{\alpha}$ we denote by $S_{\alpha}^{\beta}$ and call them canonical projections. To a decreasing sequence of elements of the covering $\Omega^{\beta}$ under a canonical projection corresponds a decreasing sequence of elements of the covering $\Omega^{\alpha}$ and we have the following homomorphisms:

$1^{\circ}$. The homomorphic mapping $\rho_{\alpha}^{\beta}$ of the group $L^{r}\left(\Omega^{\beta}, \Xi\right)$ into the group $L^{r}\left(\Omega^{\alpha}, \Xi\right)$ defined by the formula

$$
\rho_{\alpha}^{\beta} f_{\beta}^{r}\left(e_{i_{0}}^{\alpha}, \cdots, e_{i_{r}}^{\alpha}\right)=\sum f_{\beta}^{r}\left(e_{j_{0}}^{\beta}, \cdots, e_{j_{r}}^{\beta}\right),
$$

where the sum is extended over all $e_{j_{0}}^{\beta} \supset \ldots \supset e_{j_{r}}^{\beta}$ such that $S_{0}^{\beta} e_{j_{k}}^{\beta}=e_{i_{k}}^{\alpha}$.

$2^{\circ}$. The homomorphic mapping $\sigma_{\beta}^{\alpha}$ of the group $L^{r}\left(\Omega^{\alpha}, X\right)$ into the group $L^{r}\left(\Omega^{\beta}, X\right)$ defined by the formula

$$
\underset{\sigma_{\beta}}{\alpha} f_{\alpha}^{r}\left(e_{j_{0}}^{\beta}, \cdots, e_{j_{r}}^{\beta}\right)=f_{\alpha}^{r}\left(S_{\alpha}^{\beta} e_{j_{0}}^{\beta}, \cdots, S_{\alpha}^{\beta} e_{j_{r}}^{\beta}\right) .
$$

The homomorphisms $\rho_{\alpha}^{\beta}$ and $\sigma_{\beta}^{\alpha}$ preserve respectively the lower and the 
upper boundary operators and generate, correspondingly, the homomorphism $\tilde{\omega}_{\alpha}^{\beta}$ of the group $B_{\Delta}^{r}\left(\Omega^{\beta}, \Xi\right)$ into the group $B_{\Delta}^{r}\left(\Omega^{\alpha}, \Xi^{\prime}\right)$ and the homomorphism $\pi_{\beta}^{\alpha}$ of the group $B_{\nabla}^{r}\left(\Omega^{\alpha}, X\right)$ into the group $B_{\nabla}^{r}\left(\Omega^{\beta}, X\right)$.

If $\Xi$ is, as always, a bicompact topological group, then the groups $B_{\Delta}^{r}\left(\Omega^{\alpha}, \Xi\right)$ are also bicompact and we have the inverse spectrum

$$
\left[B_{\Delta}^{r}\left(\Omega^{\alpha}, \Xi\right) ; \tilde{\omega}_{\alpha}^{\beta}\right]
$$

with a bicompact limit group $B_{\Delta}^{r}(R, \Xi)$ called the $r$-dimensional $\Delta$-group of the space $R$ to the field of coefficients $\Xi$.

Let $X$ be a discrete group; the limit group of the direct spectrum( $\left.{ }^{10}\right)$

$$
\left[B_{\nabla}^{r}\left(\Omega^{\alpha}, X\right) ; \pi_{\beta}^{\alpha}\right]
$$

is called the $r$-dimensional $\nabla$-group of the space $R$ to the field of coefficients $X$ and is denoted by $B_{\nabla}^{r}(R, X)$.

9.41. If $X$ and $\Xi$ are dual groups and $X$ is discrete and $\Xi$ bicompact, then the groups $B_{\nabla}^{r}(R, X)$ and $B_{\Delta}^{r}(R, \Xi)$ are also dual.

9.42. In the case of a normal $R$ the just-defined Betti groups remain the same up to an isomorphism, if instead of the system of all open coverings of the space $R$ we consider the system of all closed coverings.

The proposition 9.41 follows from Theorem 6.1 of Steenrod's paper cited in footnote 4.

The proposition 9.42 may be deduced by the following considerations. The equivalence of the two definitions of Betti groups, namely of the definition given in the present paragraph and the definition given in $\$ 7$, is proved in the case of open coverings precisely in the same way as in the case of closed coverings; indeed, in the present paragraph we made nowhere use of the fact that the coverings $\Omega^{\alpha}$ consist of open sets. But as regards the equivalence of the definition given in $\$ 7$ with the analogous definition based on closed coverings, it was proved by Čech $\left({ }^{7}\right)$.

Remark. If the multiplicative coverings $\Omega^{\gamma}, \Omega^{\beta}, \Omega^{\alpha}$ follow one after another,

$$
\Omega^{\gamma}>\Omega^{\beta}>\Omega^{\alpha},
$$

and $S_{\beta}^{\gamma}, S_{\alpha}^{\beta}, S_{\alpha}^{\gamma}$ denote the corresponding canonical projections, then the equality

$$
S_{\alpha}^{\beta} S_{\beta}^{\gamma}=S_{\alpha}^{\gamma}
$$

may not be true in spite of the fact that (9.393) and (9.394) always hold.

(10) Here again the definition of the limit-group can be given in a simplified form analogous to that of 4.8 . 
However, it may be easily shown that the equality (9.4) always holds, if $\Omega^{\beta}$ is a so-called exact subdivision of $\Omega^{\alpha}$ and $\Omega^{\gamma}$ is an exact subdivision of $\Omega^{\beta}$. We say that a subdivision $\Omega^{\beta}$ of the covering $\Omega^{\alpha}$ is an exact subdivision if it satisfies the following condition: for any $e_{i}^{\alpha} \varepsilon \Omega^{\alpha}, e_{j}^{\beta} \varepsilon \Omega^{\beta}$, the set $e_{i}^{\alpha} n e_{j}^{\beta}$ is an element of the covering $\Omega^{\beta}$.

In the first place it is easy to prove that if $\Omega^{\gamma}$ is an exact subdivision of $\Omega^{\beta}$ and $\Omega^{\beta}$ is an exact subdivision of $\Omega^{\alpha}$, then $\Omega^{\gamma}$ is an exact subdivision of $\Omega^{\alpha}$. In fact, let $e_{i}^{\alpha} \varepsilon \Omega^{\alpha}$ and $e_{k}^{\gamma} \varepsilon \Omega^{\gamma}$ be chosen arbitrarily. Take any $e_{j}^{\beta} \supset e_{k}^{\gamma}$. Then

$$
e_{i}^{\alpha} \cap e_{k}^{\gamma}=e_{i}^{\alpha} \cap e_{j}^{\beta} \cap e_{k}^{\gamma}=e_{h}^{\beta} n e_{k}^{\gamma}=e_{k}^{\gamma} \text {. }
$$

For the proof of the equality (9.4) in the case of exact subdivisions consider some $e_{k}^{\gamma} \varepsilon \Omega^{\gamma}$ and put

$$
S_{\beta}^{\gamma} e_{k}^{\gamma}=e_{j}^{\beta}, \quad S_{\alpha}^{\beta} e_{j}^{\beta}=e_{i}^{\alpha}, \quad S_{\alpha}^{\gamma} e_{k}^{\gamma}=e_{h}^{\alpha} .
$$

Evidently $e_{h}^{\alpha} \subset e_{i}^{\alpha}$ and hence it is sufficient to show that $e_{i}^{\alpha} \subset e_{h}^{\alpha}$. To this end consider the set $e_{j}^{\beta} \cap e_{h}^{\alpha}$. Since $\Omega^{\beta}$ is an exact subdivision of $\Omega^{\alpha}$, we have

$$
\stackrel{\beta}{e_{j}} \cap \stackrel{\alpha}{e_{h}}=e_{l}^{\beta} \varepsilon \Omega^{\beta},
$$

and (since $S_{\beta}^{\gamma} e_{k}^{\gamma}=e_{j}^{\beta}, e_{k}^{\gamma} \subset e_{l}^{\beta}$ )

$$
e_{j}^{\beta} \cap e_{l}^{\beta}=e_{j}^{\beta},
$$

i.e.,

$$
e_{j}^{\beta} \subset e_{l}^{\beta}
$$

On the other hand

$$
e_{l}^{\beta}=\stackrel{\beta}{e_{j}} \cap \stackrel{\alpha}{e_{h}} \subset e_{j}^{\beta} \cap \stackrel{\alpha}{e_{i}}=e_{j}^{\beta}
$$

hence

$$
\stackrel{\beta}{e_{j}}=e_{l}^{\beta} \subset \stackrel{\alpha}{\alpha},
$$

and consequently $e_{i}^{\alpha} \subset e_{h}^{\alpha}$, q.e.d.

Observe that any two multiplicative coverings $\Omega^{\alpha}$ and $\Omega^{\beta}$ have a common exact subdivision $\Omega^{\gamma}$. In fact, it is sufficient to take for $\Omega^{\gamma}$ the covering consisting of all sets of the form $e_{i}^{\alpha} n e_{j}^{\beta}$, where $e_{i}^{\alpha} \varepsilon \Omega^{\alpha}$ and $e_{j}^{\beta} \varepsilon \Omega^{\beta}$.

\section{The CONNECTIVITY RING}

10.1. The definition of the Betti groups given in the preceding paragraph enables us to transfer to these groups the operation of multiplication defined by Alexander $\left({ }^{6}\right)$. The advantage of the so-obtained theory in comparison with Alexander's theory consists in the independence of our constructions from any arbitrary ordering of the vertices. 
Let then be given a commutative ring $J$ (the ring of coefficients), a multiplicative covering

$$
\Omega=\left\{e_{i}\right\}, \quad i=1,2, \cdots, s,
$$

of the space $R$ and two functions $f^{p}\left(e_{i_{0}}, \cdots, e_{i_{p}}\right)$ and $f^{q}\left(e_{i_{0}}, \cdots, e_{i_{q}}\right)$ satisfying the conditions of 9.4 . Construct the function $f^{p+q}=\left[f^{p} \cdot f^{q}\right]$ putting for any decreasing sequence

$$
e_{i_{0}} \supset \cdots \supset e_{i_{+}}
$$

of $p+q+1$ elements of the covering $\Omega$

$$
f^{p+q}\left(e_{i_{0}}, \cdots, e_{i_{p} q}\right)=f^{p}\left(e_{i_{0}}, \cdots, e_{i_{p}}\right) f^{q}\left(e_{i_{p}}, \cdots, e_{i_{p} q}\right) .
$$

In the same way as Alexander we deduce by means of simple computation that this multiplication is associative and distributive with respect to addition and that it possesses the following fundamental property:

10.11. $\nabla\left[f^{p} \cdot f^{q}\right]=\left[\nabla f^{p} \cdot f^{q}\right]+(-1)^{p}\left[f^{p} \cdot \nabla f^{q}\right]$.

From 10.11 immediately follows

10.12. The product of two cycles is a cycle.

10.13. The product of any cycle with a cycle homologous to zero is equal to zero.

Hence in its turn it follows that the operation of multiplication of functions generates the operation of multiplication of elements of Betti groups: if $z^{p} \varepsilon B_{\nabla}^{p}(\Omega, J), z^{q} \varepsilon B_{\nabla}^{q}(\Omega, J)$, then by $\left[z^{p} \cdot z^{q}\right]$ we denote the class of homologies $z^{p+q} \varepsilon B^{p+q}(\Omega, J)$ containing the cycle $f^{p+q}=\left[f^{p} \cdot f^{q}\right]$, where $f^{p}$ and $f^{q}$ are arbitrary cycles belonging respectively to the homological classes $z^{p}$ and $z^{q}$.

10.2. Let now be given two elements of the groups $B_{\nabla}^{p}(R, J)$ and $B_{\nabla}^{q}(R, J)$, i.e., two bundles $u^{p}$ and $u^{q}$ of the spectra

$$
\left[B_{\nabla}^{p}\left(\Omega^{\alpha}, J\right) ; \pi_{\beta}^{\alpha}\right], \quad\left[B_{\nabla}^{q}\left(\Omega^{\alpha}, J\right) ; \pi_{\beta}^{\alpha}\right] .
$$

Choose in every bundle an element, $u_{\alpha}^{p} \varepsilon u^{p}, u_{\alpha}^{q} \varepsilon u^{q}$ (with the same $\alpha$ in both cases) and denote by $\left[u^{p} \cdot u^{q}\right]$ the bundle $u^{p+q} \varepsilon B_{\nabla}^{p+q}(R, J)$ containing the element $\left[u_{\alpha}^{p} \cdot u_{\alpha}^{q}\right]$.

Let us prove that the so-defined product $\left[u^{p} \cdot u^{q}\right]$ does not depend on the choice of the elements $u_{\alpha}^{p} \varepsilon u^{p}$ and $u_{\alpha}^{q} \varepsilon u^{q}$. To this end we prove in the first place

10.21. If $\Omega^{\beta}>\Omega^{\alpha}$ and $\sigma_{\beta}^{\alpha}$ is a mapping of the group $L^{r}\left(\Omega^{\alpha}\right)$ into the group $L^{r}\left(\Omega^{\beta}\right)$ generated by some projection $S_{\alpha}^{\beta}$ of the covering $\Omega^{\beta}$ into the covering $\Omega^{\alpha}$, then

$$
\sigma_{\beta}^{\alpha}\left[f_{\alpha}^{p} \cdot f_{\alpha}^{q}\right]=\left[\begin{array}{c}
\alpha \\
\left.\sigma_{\beta} f_{\alpha}^{p} \cdot \sigma_{\beta}^{\alpha} f_{\alpha}^{q}\right]
\end{array}\right.
$$


In fact,

$$
\begin{aligned}
\sigma_{\beta}^{\alpha}\left[f_{\alpha}^{p} \cdot f_{\alpha}^{q}\right]\left(e_{j_{0}}^{\beta} \cdots e_{j_{+q} q}^{\beta}\right) & =\left[f_{\alpha}^{p} \cdot f_{\alpha}^{q}\right]\left(S_{\alpha}^{\beta} e_{j_{0}}^{\beta} \cdots S_{\alpha}^{\beta} e_{j_{p+q}}^{\beta}\right) \\
& =f_{\alpha}^{p}\left(S_{\alpha}^{\beta} e_{j_{0}}^{\beta}, \cdots, S_{\alpha}^{\beta} e_{j_{p}}^{\beta}\right) f_{\alpha}^{q}\left(S_{\alpha}^{\beta} e_{j_{p}}^{\beta}, \cdots, S_{\alpha}^{\beta} e_{j_{p+q}}^{\beta}\right) \\
& =\sigma_{\beta}^{\alpha} f_{\alpha}^{p}\left(e_{j_{0}}^{\beta}, \cdots, e_{j_{p}}^{\beta}\right) \sigma_{\beta}^{\alpha} f_{\alpha}^{q}\left(e_{j_{p}}^{\beta}, \cdots, e_{j_{p+q}}^{\beta}\right) \\
& =\left[\sigma_{\beta}^{\alpha} f_{\alpha}^{p} \cdot \sigma_{\beta}^{\alpha} f_{\alpha}^{q}\right]\left(e_{j_{0}}^{\beta} \cdots e_{j_{p+q}}^{\beta}\right) .
\end{aligned}
$$

From 10.21 follows immediately

10.22. $\pi_{\beta}^{\alpha}\left[u_{\alpha}^{p} \cdot u_{\alpha}^{q}\right]=\left[\pi_{\beta}^{\alpha} u_{\alpha}^{p} \cdot \pi_{\beta}^{\alpha} u_{\alpha}^{q}\right]$.

Let now beside $u_{\alpha}^{p} \varepsilon u^{p}$ and $u_{\alpha}^{q} \varepsilon u^{q}$ be chosen $u_{\beta}^{p} \varepsilon u^{p}$, $u_{\beta}^{q} \varepsilon u^{q}$. We shall prove that $\left[u_{\alpha}^{p} \cdot u_{\alpha}^{q}\right]$ and $\left[u_{\beta}^{p} \cdot u_{\beta}^{q}\right]$ belong to one and the same bundle.

From our assumptions follows the existence of such a covering $\Omega^{r}$ that $\Omega^{\gamma}>\Omega^{\alpha}, \Omega^{\gamma}>\Omega^{\beta}$ and

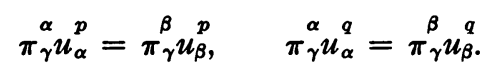

Hence, on ground of 10.22 ,

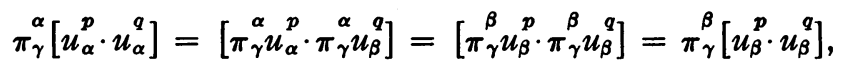

whence indeed it follows that $\left[u_{\alpha}^{p} \cdot u_{\alpha}^{q}\right]$ and $\left[u_{\beta}^{p} \cdot u_{\beta}^{q}\right]$ belong to one and the same bundle.

Definition 10.23. The direct sum of the groups $B_{\nabla}^{r}(R, J), r=0,1,2, \cdots$, is denoted by $B_{\nabla}^{r}(R, J)$ and is called the complete Betti $\nabla$-group of the space $R$.

From what has been proved it follows that the established operation of multiplication of elements of the group $B_{\nabla}(R, J)$ transforms this group into a commutative ring. This ring is called the connectivity ring of the space $R$.

AdDendum. On CERTAIN PROPOSITIONS OF THE THEORY OF GROUPS

1. The theory of characters of commutative groups is taken for granted in the present paper.

If of two groups $X$ and $\Xi$, of which $X$ is discrete and $\Xi$ is bicompact, one is the group of characters of the other, then the groups $X$ and $\Xi$ are called dual or conjugated. For any $x \varepsilon X, \xi \varepsilon \Xi$ we have in this case that

$$
\xi(x)=x(\xi)
$$

is en element of the group $\kappa$ denoted by $\xi x=x \xi$. The group of characters of a group $G$ we denote by $a G$.

2. Let there be given two groups $A^{\alpha}$ and $A^{\beta}$ both discrete and both bicompact; their groups of characters we denote by $X^{\alpha}$ and $X^{\beta}$. Let there be given a homomorphic mapping $\phi_{\alpha}^{\beta}$ of the group $A^{\beta}$ into the group $A^{\alpha}$. To 
every element $x_{\alpha} \varepsilon X^{\alpha}$ we correlate in the following manner an element $x_{\beta}=f_{\beta}^{\alpha} x_{\alpha}$ of the group $X^{\beta}$ : by definition, the character $x_{\beta} \varepsilon X^{\beta}$ of the group $A^{\beta}$ maps every element $a_{\beta} \varepsilon A^{\beta}$ on the element $x_{\alpha} \phi_{\alpha}^{\beta} a_{\beta}$ of the group $\kappa$. In other words, the character $x_{\beta}=f_{\beta}^{\alpha} x_{\alpha}$ of the group $A^{\beta}$ is determined by the equation

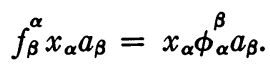

The mapping $\phi_{\alpha}^{\beta}$ of the group $A^{\beta}$ into the group $A^{\alpha}$ and the mapping $f_{\beta}^{\alpha}$ of the group $X^{\alpha}$ into the group $X^{\beta}$ are called conjugated mappings. The relation of conjugateness of two mappings is a symmetrical relation.

TheOREM I. Let there be given two isomorphic groups $A^{\alpha}$ and $A^{\beta}$; denote by $\phi_{\alpha}^{\beta}$ any isomorphic mapping of $A^{\beta}$ on $A^{\alpha}$. Then the homomorphism $f_{\beta}^{\alpha}$ conjugated to the isomorphism $\phi_{\alpha}^{\beta}$ is an isomorphic mapping of the group $X^{\alpha}=\chi A^{\alpha}$ on the group $X^{\beta}=\chi A^{\beta}$.

Proof. The mapping $f_{\beta}^{\alpha}$ is defined by the formula (1); in order to prove that $f_{\beta}^{\alpha}$ is an isomorphic mapping it is sufficient to show that for $x_{\alpha} \varepsilon X^{\alpha}$, $x_{\alpha} \neq 0$, we have also $f_{\beta}^{\alpha} x_{\alpha} \neq 0$, i.e., that at least for one $a_{\beta} \varepsilon A^{\beta}$

$$
f_{\beta}^{\alpha} x_{\alpha} a_{\beta} \neq 0 \text {. }
$$

Since $x_{\alpha} \neq 0$, there exists such an $a_{\alpha} \varepsilon A^{\alpha}$ that $x_{\alpha} a_{\alpha} \neq 0$. Since $\phi_{\alpha}^{\beta}$ is an isomorphism, there exists such an $a_{\beta} \neq 0$ (and, moreover, a unique one) that $\phi_{\alpha}^{\beta} a_{\beta}=a_{\alpha}$. Then

$$
f_{\beta}^{\alpha} x_{\alpha} a_{\beta}=x_{\alpha} \phi_{\alpha}^{\beta} a_{\beta}=x_{\alpha} a_{\alpha} \neq 0,
$$

and our assertion is proved.

Thus $f_{\beta}^{\alpha}$ is an isomorphism. Let us prove that $f_{\beta}^{\alpha}$ maps $X^{\alpha}$ on $X^{\beta}$. Let there be given $x_{\beta} \varepsilon X^{\beta}, x_{\beta} \neq 0$. We have to find an $x_{\alpha} \varepsilon X^{\alpha}$ such that for any $a_{\beta} \varepsilon A^{\beta}$

$$
f_{\beta}^{\alpha} x_{\alpha} a_{\beta}=x_{\alpha} \phi_{\alpha}^{\beta} a_{\beta}=x_{\beta} a_{\beta} .
$$

Since $\phi_{\alpha}^{\beta}$ is an isomorphism on $A^{\alpha}$, for every $a_{\alpha} \varepsilon A^{\alpha}$ there exists a unique $a_{\beta} \varepsilon A^{\beta}$ such that $\phi_{\alpha}^{\beta} a_{\beta}=a_{\alpha}$. Putting

$$
x_{\alpha} a_{\alpha}=x_{\beta} a_{\beta},
$$

we determine the required $x_{\alpha}$.

Theorem I is thus proved.

THEOREM II. Let there be given two groups $A^{\alpha}$ and $A^{\beta}$ respectively dual to the groups $X^{\alpha}$ and $X^{\beta}$, an isomorphism $\phi_{\alpha}^{\beta}$ of the group $A^{\beta}$ on $A^{\alpha}$ and the isomorphism $\phi_{\beta}^{\alpha}$ of the group $A^{\alpha}$ on $A^{\beta} d$ ual to the isomorphism $\phi_{\alpha}^{\beta}$ :

$$
\stackrel{\alpha}{\phi_{\beta}^{\alpha}}=\left(\phi_{\alpha}^{\beta}\right)^{-1} \text {. }
$$


Denote by $f_{\beta}^{\alpha}$ and $f_{\alpha}^{\beta}$ the isomorphisms conjugated to the isomorphisms $\phi_{\alpha}^{\beta}$ and $\phi_{\beta}^{\alpha}$. Then

$$
f_{\alpha}^{\beta}=\left(f_{\beta}^{\alpha}\right)^{-1} \text {. }
$$

Proof. The isomorphisms $f_{\beta}^{\alpha}$ and $f_{\alpha}^{\beta}$ are determined by the equations

$$
f_{\beta}^{\alpha} x_{\alpha} a_{\beta}=x_{\alpha} \phi_{\alpha}^{\beta} a_{\beta}, \quad f_{\alpha}^{\beta} x_{\beta} a_{\alpha}=x_{\beta} \phi_{\beta}^{\alpha} a_{\alpha} .
$$

Let

$$
a_{\beta}=\phi_{\beta}^{\alpha} a_{\alpha}=\left(\phi_{\alpha}^{\beta}\right)^{-1} a_{\alpha}, \quad x_{\beta}=f_{\beta}^{\alpha} x_{\alpha} .
$$

For any $a_{\alpha} \varepsilon A^{\alpha}$ and any $x_{\beta} \varepsilon f_{\beta}^{\alpha} x_{\alpha} \varepsilon X^{\beta}$ we have

$$
\begin{gathered}
f_{\alpha}^{\beta} x_{\beta} a_{\alpha}=x_{\beta} \phi_{\beta}^{\alpha} a_{\alpha}=x_{\beta} a_{\beta}=f_{\beta}^{\alpha} x_{\alpha} a_{\beta}=x^{\alpha} \phi_{\alpha}^{\beta} a_{\beta}=x_{\alpha} a_{\alpha}, \\
\left(f_{\beta}^{\alpha}\right)^{-1} x_{\beta} a_{\beta}=\left(f_{\beta}^{\alpha}\right)^{-1} f_{\beta}^{\alpha} x_{\alpha} a_{\alpha}=x_{\alpha} a_{\alpha},
\end{gathered}
$$

i.e.,

$$
f_{\alpha}^{\beta} x_{\beta} a_{\alpha}=\left(f_{\beta}^{\alpha}\right)^{-1} x_{\beta} a_{\alpha}
$$

q.e.d.

THEOREM III. Let the groups

$$
X^{\alpha}, X^{\beta}, X^{1 \alpha}, X^{1 \beta}
$$

be dual respectively to the groups

$$
A^{\alpha}, A^{\beta}, A^{1 \alpha}, A^{1 \beta} \text {. }
$$

Let there be given isomorphic mappings $\rho_{\alpha}^{1 \alpha}$ and $\rho_{\beta}^{1 \beta}$ of $A^{1 \alpha}$ and $A^{1 \beta}$ on $A^{\alpha}$ and $A^{\beta}$ respectively. The conjugated isomorphisms we denote by $\sigma_{1 \alpha}^{\alpha}$ and $\sigma_{1 \beta}^{\beta}$. Let there be given, besides, homomorphic mappings $\tilde{\omega}_{\alpha}^{\beta}$ and $\tilde{\omega}_{1 \alpha}^{1 \beta}$ correspondingly of $A^{\beta}$ into $A^{\alpha}$ and $A^{1 \beta}$ into $A^{1 \alpha}$, and the conjugated homomorphisms $\pi_{\beta}^{\alpha}$ and $\pi_{1 \beta}^{1 \alpha}$. Let it be known that

$$
\tilde{\omega}_{1 \alpha}^{1 \beta}=\left(\rho_{\alpha}^{1 \alpha}\right)^{-1} \tilde{\omega}_{\alpha \rho_{\beta} \beta}^{1 \beta}
$$

Then

$$
\pi_{1 \beta}^{1 \alpha}=\sigma_{1 \beta}^{\beta} \pi_{\beta}^{\alpha}\left(\sigma_{1 \alpha}^{\alpha}\right)^{-1}
$$

Proof. For the proof it suffices to show that

$$
\underset{\sigma_{1 \beta} \pi_{\beta}^{\alpha}\left(\sigma_{1 \alpha}^{\alpha}\right)^{-1}}{\alpha}
$$

satisfies the functional equation

$$
\tilde{\pi}_{1 \beta}^{1 \alpha} x_{1 \alpha} a_{1 \beta}=x_{1 \alpha} \tilde{\omega} \tilde{\omega}_{1 \alpha}^{1 \beta} a_{1 \beta}
$$


determining $\pi_{1 \beta}^{1 \alpha}$, i.e., it is sufficient to prove that for any $x_{1 \alpha}$ and $a_{1 \beta}$ we have

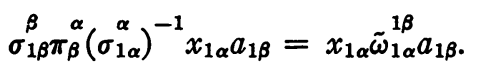

Let us prove this. To this end, replacing $\left(\sigma_{1 \alpha}^{\alpha}\right)^{-1} x_{1 \alpha}$ by $x_{\alpha}$ and $\tilde{\omega}_{1 \alpha}^{1 \beta}$ by its expression (2), we write (3) in the form

$$
\underset{\sigma_{1 \beta} \pi_{\beta}^{\alpha} x_{\alpha} a_{1 \beta}}{\beta}=x_{1 \alpha}\left(\rho_{\alpha}^{1 \alpha}\right)^{-1} \tilde{\omega}_{\alpha \rho_{\beta}}^{\beta \beta} a_{1 \beta} .
$$

But $\left(\rho_{\alpha}^{1 \alpha}\right)^{-1}$ and $\left(\sigma_{1 \alpha}^{\alpha}\right)^{-1}$ are conjugated isomorphisms and hence

$$
\left(\sigma_{1 \alpha}^{\alpha}\right)^{-1} x_{1 \alpha} a_{\alpha}=x_{1 \alpha}\left(\rho_{\alpha}^{1 \alpha}\right)^{-1} a_{\alpha} .
$$

Thus

$$
x_{1 \alpha}\left(\rho_{\alpha}^{1 \alpha}\right)^{-1} a_{\alpha}=\left(\sigma_{1 \alpha}^{\alpha}\right)^{-1} x_{1 \alpha} a_{\alpha}=x_{\alpha} a_{\alpha} .
$$

Substituting this into (4), we obtain as the equality to be proved the following:

$$
\underset{\sigma_{1 \beta} \pi_{\beta}^{\alpha} x_{\alpha} a_{1 \beta}}{\beta}=x_{\alpha} \tilde{\omega}_{\alpha}^{\beta} \rho_{\beta}^{1 \beta} a_{1 \beta}
$$

But

$$
\stackrel{\alpha}{\pi_{\beta} x_{\alpha} a_{\beta}}=x_{\alpha} \tilde{\omega}_{\alpha}^{\beta} a_{\beta}, \quad \stackrel{\alpha}{\pi_{\beta} x_{\alpha}}=x_{\alpha} \tilde{\omega}_{\alpha}^{\beta} \varepsilon X^{\beta}
$$

and $\sigma_{1 \beta}^{9}$ and $\rho_{\beta}^{13}$ are conjugated isomorphisms. Therefore the left-hand side of the equality (5) may be transformed to the form

$$
\stackrel{\beta}{\sigma_{1 \beta} x_{\alpha} \tilde{\omega}_{\alpha}^{\beta}} a_{1 \beta}=x_{\alpha} \tilde{\omega}_{\alpha}^{\beta} \rho_{\beta}^{\beta \beta} a_{1 \beta},
$$

i.e., may be brought to coincidence with the right-hand side of the same equality. The equality (5) and Theorem III are thus proved.

Moscow, U.S.S.R. 